\title{
Republic of Mozambique: Staff Report for the 2011 Article IV Consultation, Second Review Under the Policy Support Instrument, and Request for Modification of Assessment Criteria-Staff Report; Staff Supplement; Public Information Notice and Press Release on the Executive Board Discussion; and Statement by the Executive Director for the Republic of Mozambique.
}

Under Article IV of the IMF's Articles of Agreement, the IMF holds bilateral discussions with members, usually every year. In the context of a combined discussion of the 2011 Article IV consultation with the Republic of Mozambique and the Second Review Under the Policy Support Instrument, and Request for Modification of Assessment Criteria, the following documents have been released and are included in this package:

- $\quad$ The staff report for the combined 2011 Article IV consultation and the Second Review Under the Policy Support Instrument and Request for Modification of Assessment Criteria, prepared by a staff team of the IMF, following discussions that ended on April 6, 2011, with the officials of the Republic of Mozambique on economic developments and policies. Based on information available at the time of these discussions, the staff report was completed on May 24, 2011. The views expressed in the staff report are those of the staff team and do not necessarily reflect the views of the Executive Board of the IMF.

- $\quad$ A staff supplement on the Debt Sustainability Analysis dated May 24, 2011.

- $\quad$ A Public Information Notice (PIN) and Press Release, summarizing the views of the Executive Board as expressed during its June 17, 2011, discussion of the staff report on issues related to the Article IV consultation and the IMF arrangement, respectively.

- $\quad$ A statement by the Executive Director for the Republic of Mozambique.

The documents listed below have been or will be separately released.

Letter of Intent sent to the IMF by the authorities of the Republic of Mozambique

Memorandum of Economic and Financial Policies by the authorities of the

Republic of Mozambique*

Technical Memorandum of Understanding*

*Also included in Staff Report

The policy of publication of staff reports and other documents allows for the deletion of market-sensitive information.

\author{
Copies of this report are available to the public from \\ International Monetary Fund • Publication Services \\ $70019^{\text {th }}$ Street, N.W. • Washington, D.C. 20431 \\ Telephone: (202) 623-7430 • Telefax: (202) 623-7201 \\ E-mail: publications@imf.org Internet: http://www.imf.org
}

\section{International Monetary Fund \\ Washington, D.C.}


INTERNATIONAL MONETARY FUND

REPUBLIC OF MOZAMBIQUE

\section{Staff Report for the 2011 Article IV Consultation, Second Review Under the Policy Support Instrument, and Request for Modification of Assessment Criteria}

Prepared by the African Department

(In consultation with other departments)

Approved by Roger Nord and Dominique Desruelle

May 24, 2011

- Article IV consultation and program discussions: March 23-April 6, 2011 in Maputo, with Ministers Chang (Finance), Cuereneia (Planning and Development) and other line ministers (Natural Resources, Agriculture, Trade and Industry, Energy), Bank of Mozambique (BM) Governor Gove, and other senior government officials. The mission also met with development partners and the private sector.

- Outreach: The mission conducted outreach with representatives of Parliament and civil society, including labor unions and think tanks. Following the proposal of the authorities, it traveled to Tete province in northwestern Mozambique to visit several mega-projects in the natural resource sector and the Cahora-Bassa hydroelectric power dam, discuss fiscal decentralization challenges with the provincial government, and meet with the local private sector and civil society.

- Staff: Messrs. Mueller (Head), Gitton, Rosa, Xiao (all AFR), Ms. Garcia (SPR), Mr. Lledó (resident representative), Ms. Bosten and Mr. Wane (resident representative office). Messrs. Conceição (OED), Gazel, and Kim (World Bank) joined the policy discussions.

- Focus of Article IV consultation: Discussions centered on the authorities' immediate policy priority to curb inflation and on several medium-term policy challenges: creating fiscal space to sustain investment, building more inclusive growth, and strengthening the financial sector.

- Second PSI review: Staff recommends its completion with one waiver for the nonobservance of the quantitative assessment criterion (AC) on reserve money. All structural conditionality has been observed.

- Exchange restrictions: On May 20, the authorities accepted the obligations under Article VIII of the Fund's Articles of Agreement. 


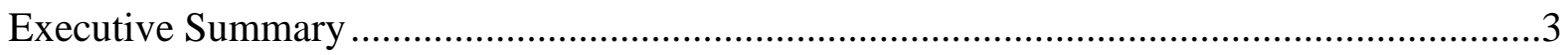

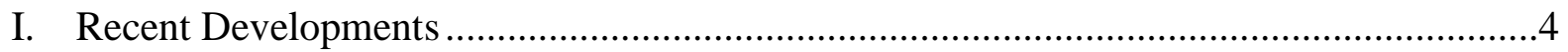

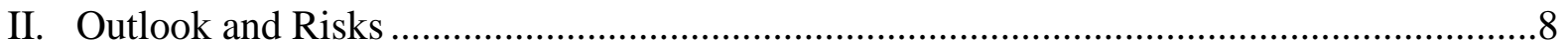

III. Short-Term Challenge: Fighting Inflation ........................................................... 10

IV. Medium-Term Challenge: Building Stable and Inclusive Growth................................14

A. Creating Fiscal Space to Sustain Investment and Support Broad-Based Growth...14

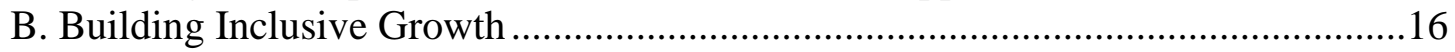

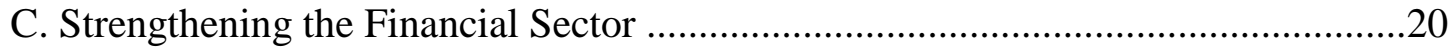

V. Mozambique's Economic Program under the PSI .................................................22

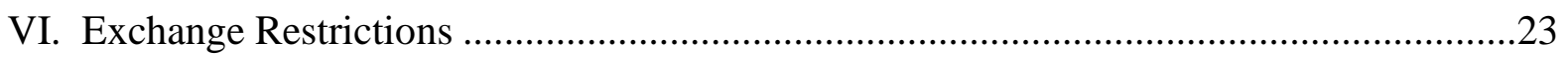

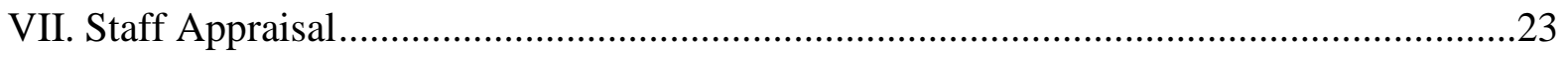

Tables

1. Selected Economic and Financial Indicators, 2009-16 ................................................26

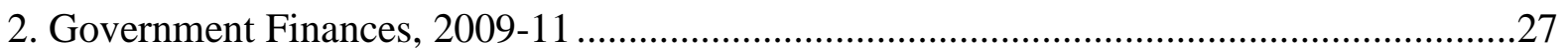

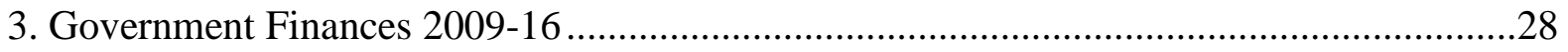

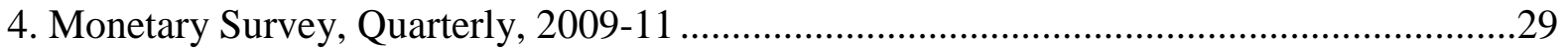

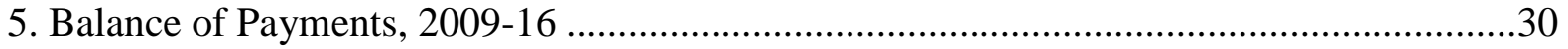

6. Financial Soundness Indicators for Banking Sector, 2001-10......................................31

7. Structural Conditionality under the Current PSI, 2010-2011 ........................................32

Figures

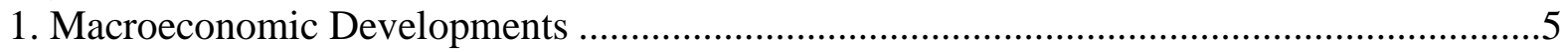

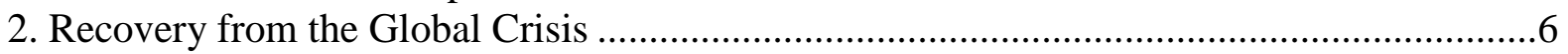

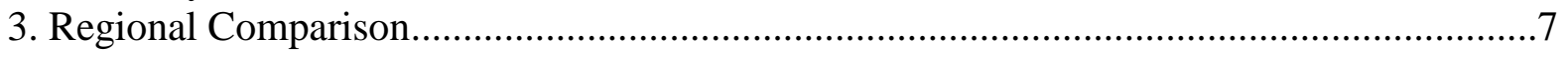

4. Monetary and Financial Sector Developments ......................................................11

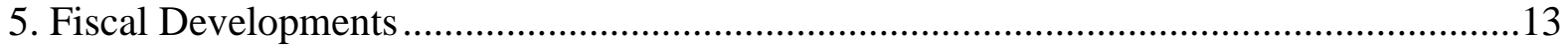

Boxes

1. Mozambique's Implementation of Past Fund Policy Recommendations ...........................4

2. Indy Village Seminar on Inclusive Growth ............................................................17

3. Action Plan for Reducing Poverty (PARP) 2011-14 ................................................18

4. Social Protection in Mozambique ........................................................................... 19

5. Fiscal Decentralization in Mozambique .....................................................................20

Appendices

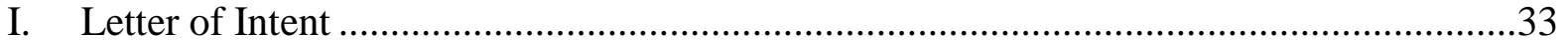

Attachment 1. Memorandum of Economic and Financial Policies ...........................34

Attachment 2. Technical Memorandum of Understanding .....................................48

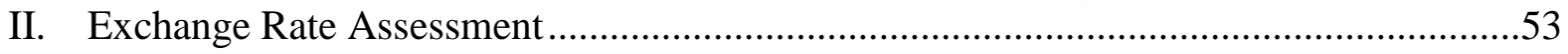

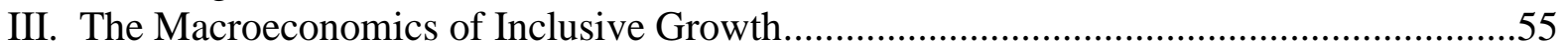

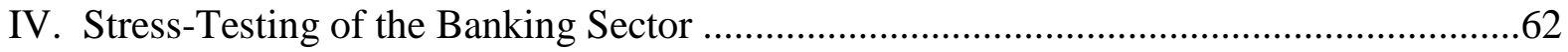




\section{EXECUTIVE SUMMARY}

Mozambique's economic performance and outlook remain strong. Helped by appropriate countercyclical policies, the authorities managed to keep economic growth buoyant during the global crisis. The country is poised for an acceleration of economic growth over the medium term, reflecting new mega-project activity in the natural resource sector and stepped-up public investment.

\section{Nonetheless, Mozambique faces important short- and medium-term policy challenges:}

- In the short run, fighting inflation is the authorities' key policy priority. Headline inflation surged in 2010, accompanied by a ratcheting up of core inflation. Since mid2010, the BM has tightened monetary policy decisively, and it intends to preserve this policy stance in 2011. Fiscal policy has been supportive of disinflation as well, as the authorities have shown spending restraint and been keeping the fiscal deficit in check, including through a revised 2011 budget law. However, given the lower domestic currency value of donor support in light of the appreciated exchange rate, the government will need to take moderate recourse to bank financing during 2011, which is not expected to crowd out private sector borrowing.

- Over the medium term, against the backdrop of stagnant poverty reduction and street riots last year, the authorities aim to adjust their growth strategy to make it more inclusive. Based on extensive deliberations with all stakeholders, including during a high-level conference on inclusive growth in February 2011, they have adopted a new Poverty Reduction Strategy (PARP) that emphasizes creating employment opportunities and continued human and social development. This strategy will complement the authorities' efforts to foster economic growth through stepped-up public investment and be embedded in their traditional policy focus on safeguarding macroeconomic stability and debt sustainability.

- Sustaining economic growth would benefit from the creation of additional fiscal space, a larger contribution of the financial sector, and implementation of key structural reforms. These efforts are embedded in the authorities' economic program supported under the PSI.

Staff recommends the completion of the second review under the PSI. All but one quantitative $\mathrm{AC}$ and all structural conditionality have been met. Staff supports the waiver for the non-observance of the end-2010 AC on reserve money, since corrective action was taken, and the proposed modifications to end-June 2011 AC in light of the authorities' revised short-term policy objectives. 


\section{RECENT DEVELOPMENTS}

\section{Mozambique's economic performance remains strong, although economic} growth is somewhat weaker than expected. A downward revision in quarterly GDP (mainly in agricultural production) suggests that the economy grew by $61 / 2$ percent in 2010 , supported by external demand. Growth remains one of the highest in the region (Figures 1 to 3). This partially reflects the authorities' supportive policy stance during the global crisis, consistent with past Fund policy advice (Box 1).

Mozambique : Comparison with Sub-Saharan Africa, Selected Indicators, 2004-10

\begin{tabular}{|c|c|c|c|c|c|c|c|c|c|c|c|c|}
\hline & \multicolumn{4}{|c|}{ Mozambique } & \multicolumn{4}{|c|}{ Sub-Saharan Africa (SSA) } & \multicolumn{4}{|c|}{ SSA - Frontier Economies ${ }^{1}$} \\
\hline & 2004-08 & 2008 & 2009 & 2010 & 2004-08 & 2008 & 2009 & 2010 & 2004-08 & 2008 & 2009 & 2010 \\
\hline Real GDP, \% change & 7.8 & 6.8 & 6.3 & 6.6 & 5.3 & 4.5 & 3.2 & 4.7 & 6.7 & 6.5 & 4.2 & 5.9 \\
\hline $\mathrm{CPI}$ period average, $\%$ change & 10.2 & 10.3 & 3.3 & 12.7 & 8.5 & 11.6 & 10.5 & 7.9 & 10.2 & 11.4 & 10.4 & 9.2 \\
\hline REER, index & 100.8 & 108.3 & 101.2 & 86.0 & 101.9 & 107.4 & 109.5 & 107.9 & 104.4 & 109.3 & 110.0 & 108.7 \\
\hline Current account balance, after grants, \% GDP & -10.9 & -11.9 & -12.1 & -10.2 & 1.0 & 1.1 & -1.9 & -1.5 & -1.6 & -3.3 & -4.4 & -4.4 \\
\hline Gross international reserves, months of imports ${ }^{2}$ & 4.8 & 4.3 & 5.4 & 5.6 & 5.2 & 5.2 & 6.0 & 5.6 & 5.2 & 4.5 & 5.5 & 5.3 \\
\hline Overall fiscal balance, including grants, \% of GDP & -2.7 & -2.2 & -5.4 & -3.7 & 1.6 & 0.2 & -5.9 & -4.6 & -1.7 & -1.8 & -5.5 & -4.5 \\
\hline Broad money (M3), \% of GDP ${ }^{2}$ & 30.3 & 33.6 & 39.8 & 40.6 & 17.3 & 19.6 & 20.7 & 21.5 & 30.6 & 32.4 & 35.7 & 36.6 \\
\hline Credit to the private sector, $\%$ of GDP & 14.4 & 18.7 & 26.5 & 28.2 & 17.1 & 19.5 & 20.7 & 21.4 & 17.4 & 19.7 & 22.5 & 22.4 \\
\hline
\end{tabular}

Source: Mozambican authorities and IMF staff estimates.

${ }^{1}$ Angola, Ghana, Kenya, Mauritius, Mozambique, Nigeria, Senegal, Tanzania, Uganda and Zambia.

${ }^{2}$ For regional comparison, months of current year imports are used. Definition of reported broad money may vary across countries.

\section{Box 1. Mozambique's Implementation of Past Fund Policy Recommendations}

Mozambique's policies have been consistent with Fund recommendations over the past years. The authorities and the staff generally agreed on the appropriate macroeconomic policy stance and the focus of the structural reform agenda.

The 2009 Article IV discussions focused on ways to mitigate the adverse impact of the global crisis and on strengthening the country's growth prospects. Consistent with Fund policy advice, the authorities took advantage of their strong policy buffers to ease fiscal and monetary policy to support economic activity in the short run. As to exchange rate policy, they also began to pay more attention to a broad basket of currencies, which resulted in larger volatility but effectively addressed the previously observed overvaluation. With a more medium-term angle, the question of sustaining Mozambique's growth momentum was tackled in the context of the design of the successor PSI program, which placed considerable emphasis on addressing infrastructure bottlenecks and implementing bold reforms to improve economic decision-making (e.g., through public financial management (PFM), debt management, and investment planning) and boost financial sector development and stability. However, as illustrated in Appendix II, the pace of structural reforms to enhance competitiveness through improvements in the business environment has been slow.

2. Inflation reached double digits. Average inflation in 2010, at $123 / 4$ percent, was in line with projections, but well above the authorities' initial objectives. End-of-period inflation fell from 161/2 percent at end-2010 to 13 percent in April 2011. However, core inflation (excluding food and fuel) has been ratcheting upward since mid-2009 and remains at 10 percent. Headline inflation was affected by exogenous factors such as global price trends, as well as the initiated reduction of the fuel subsidy which triggered price increases for gasoline and diesel of 42 and 21 percent, respectively, over the last 12 months. The rise in core inflation mirrors the lingering effects of the accommodating monetary policy stance during the global crisis and the pass-through of a sharply depreciating metical throughout much of 2010. Inflation pressures have abated little in recent months despite the recent appreciation of the metical and a tightening of macroeconomic policies. 
Figure 1. Mozambique: Macroeconomic Developments

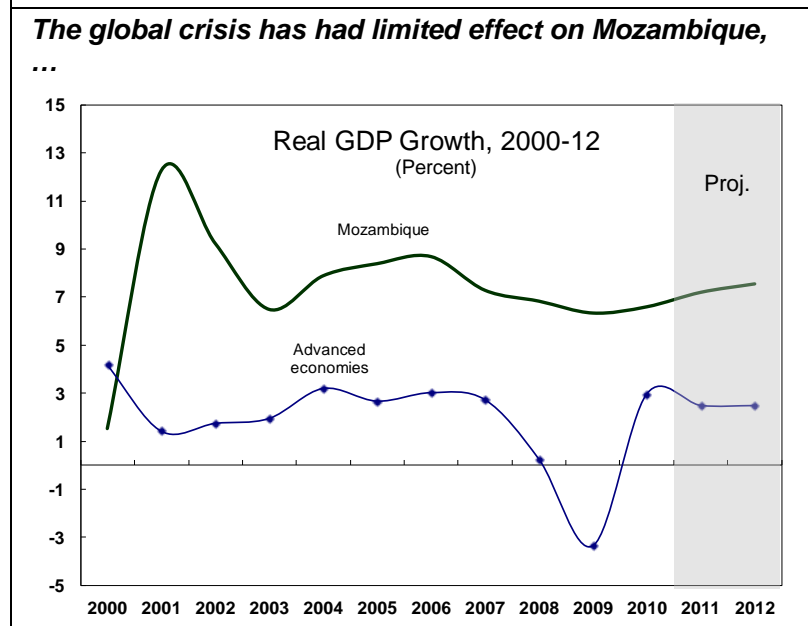

... although trend growth has declined in recent years.

Inflation picked up significantly in 2010,...
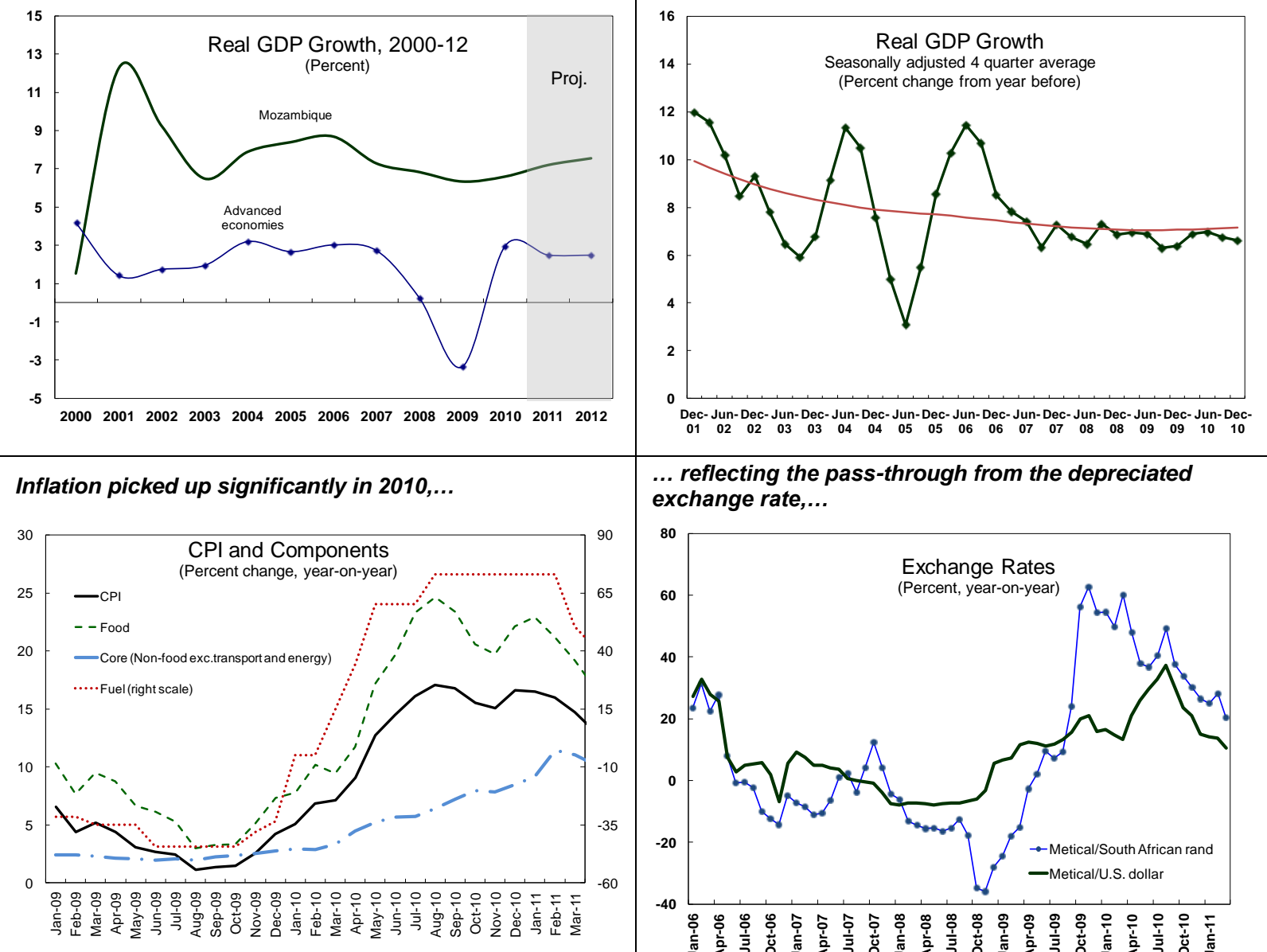

... reflecting the pass-through from the depreciated exchange rate,...

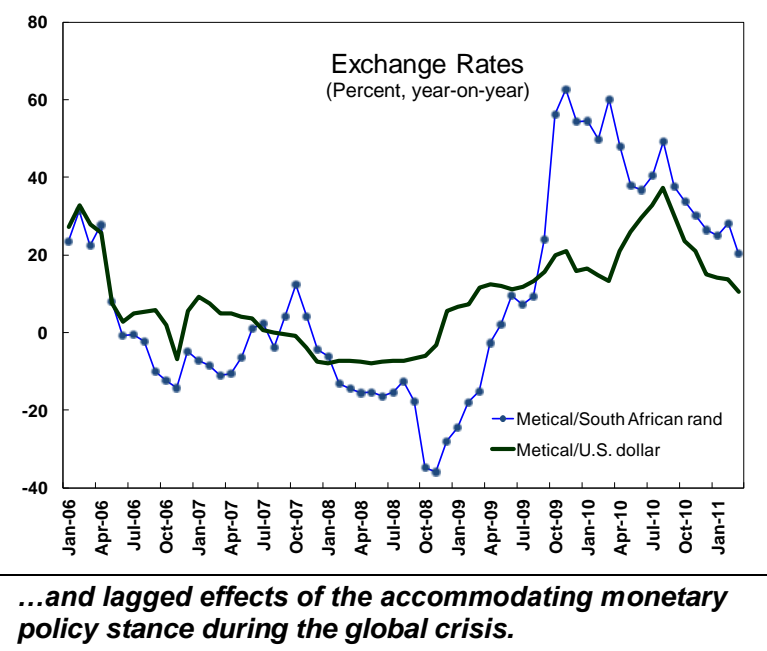

\section{... rising international food and fuel prices,...}
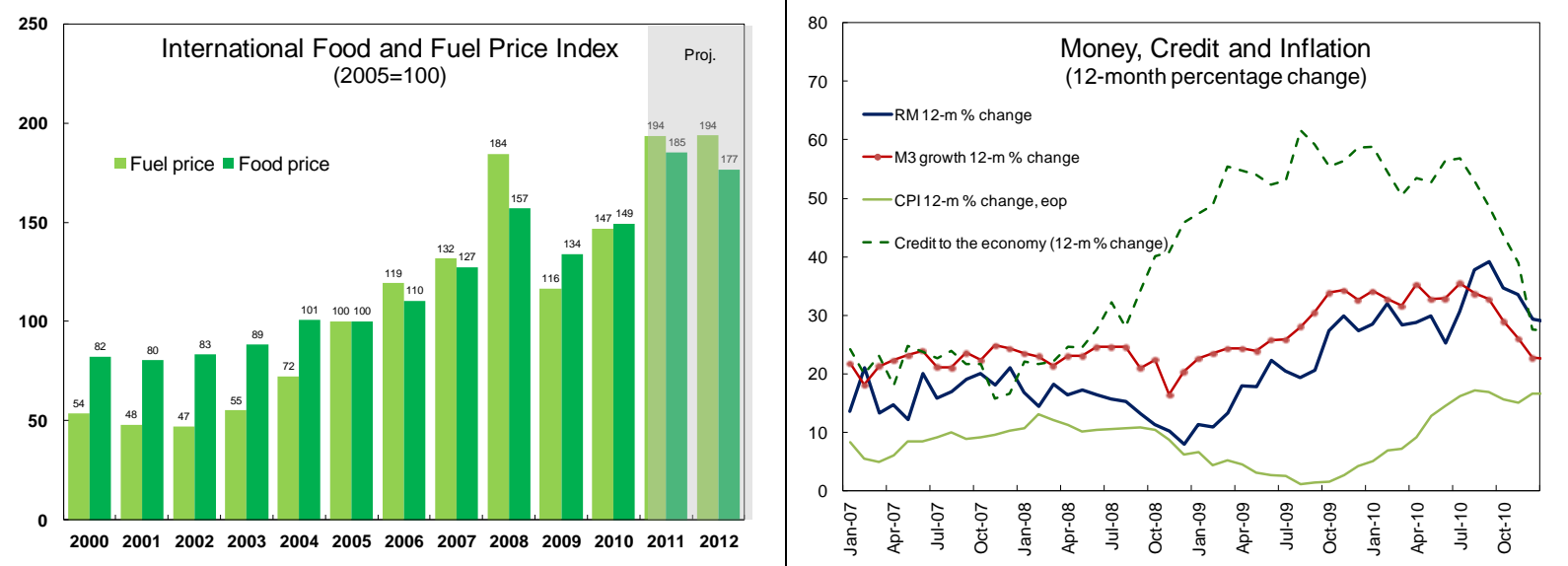

Sources: Mozambican authorities and IMF staff estimates and projections. 
Figure 2. Mozambique: Recovery from the Global Crisis

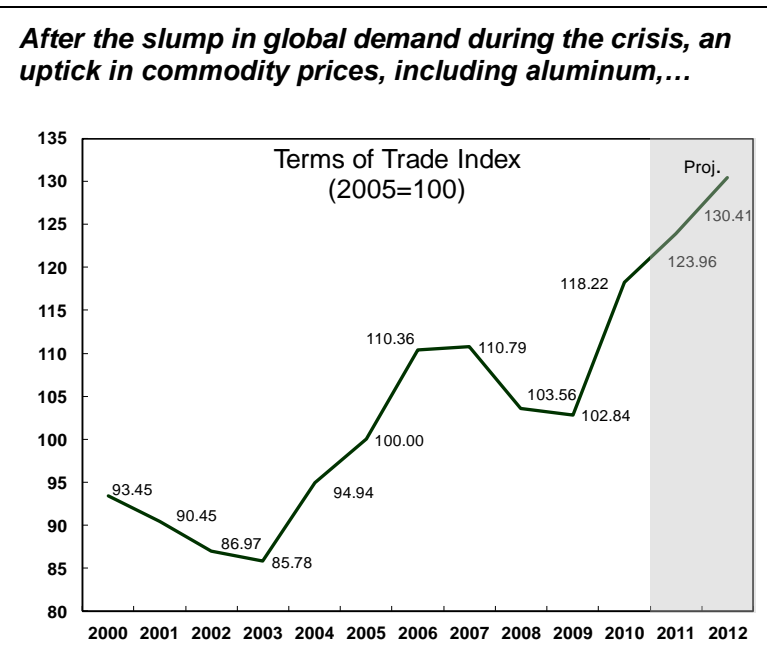
...as well as the coming online of new megaprojects, are
expected to result in a recovery of export receipts to the pre-crisis level by 2011.

However, Mozambique's traditional export sectors are lagging, mainly due to the weak business environment and marketing problems,...

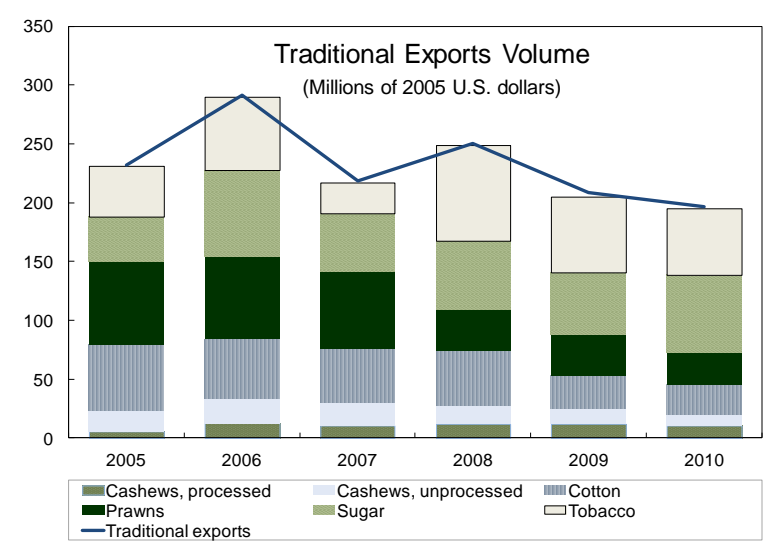

\section{The current account showed resilience to the global} crisis,...
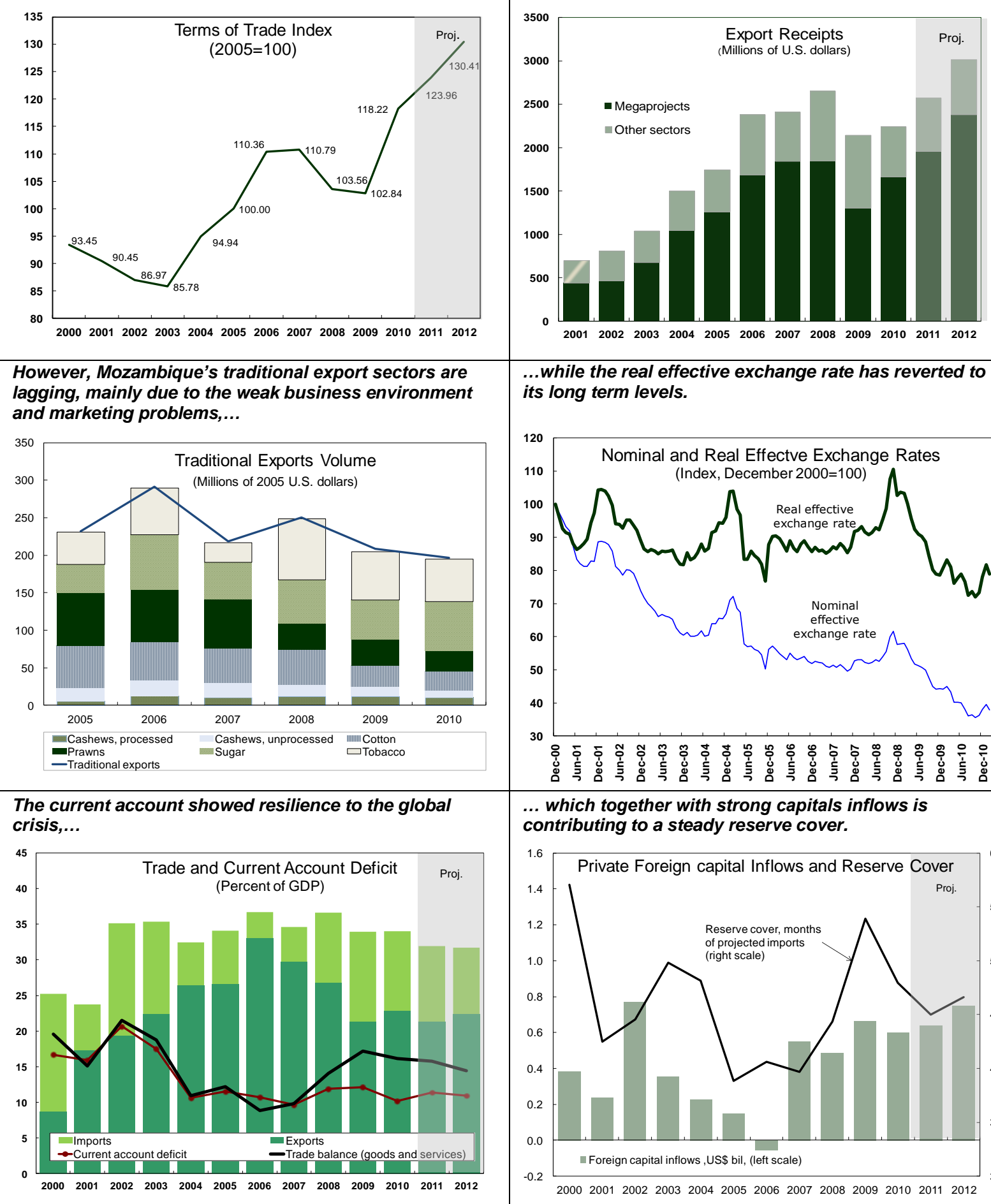

\section{...while the real effective exchange rate has reverted to} its long term levels.

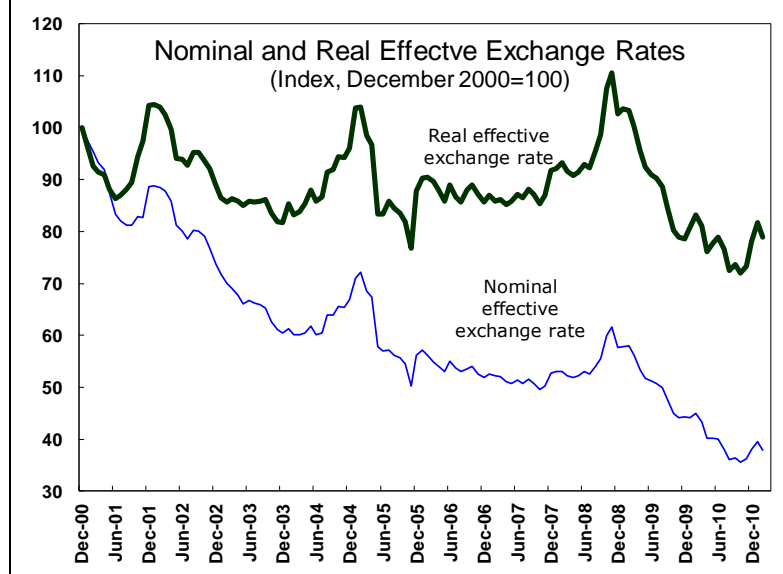

... which together with strong capitals inflows is contributing to a steady reserve cover.

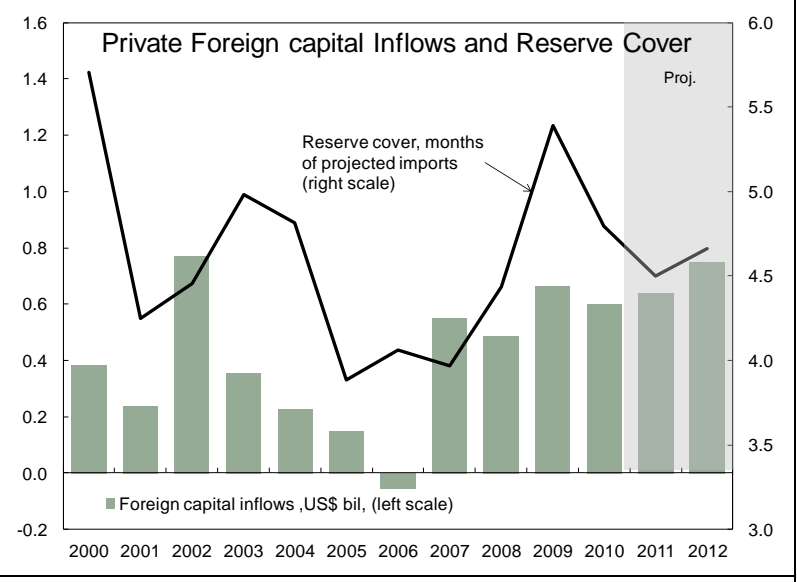

Sources: Mozambican authorities and IMF staff estimates and projections. 
Figure 3. Mozambique: Regional Comparison

Mozambique's real growth held up well relative to that in its peers in the SSA frontier markets and the SSA region.

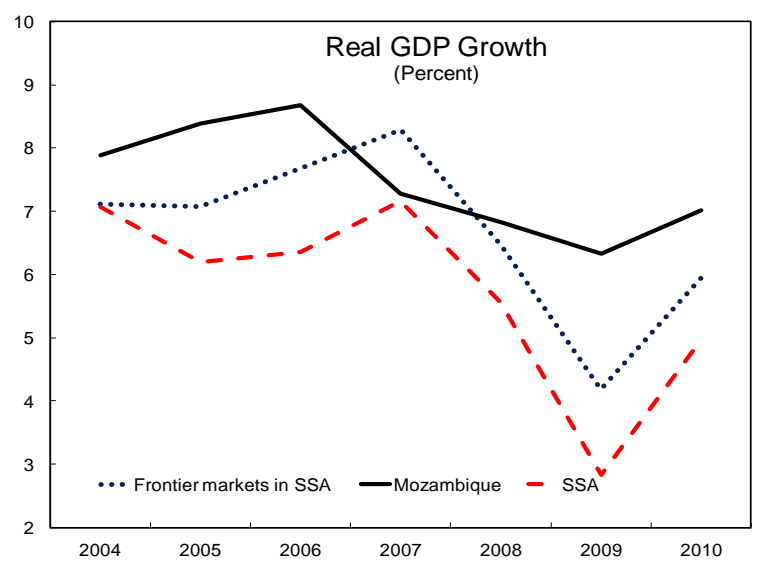

Similar to its peers, Mozambique's fiscal position strengthened somewhat in 2010 following the large stimulus in the previous year.

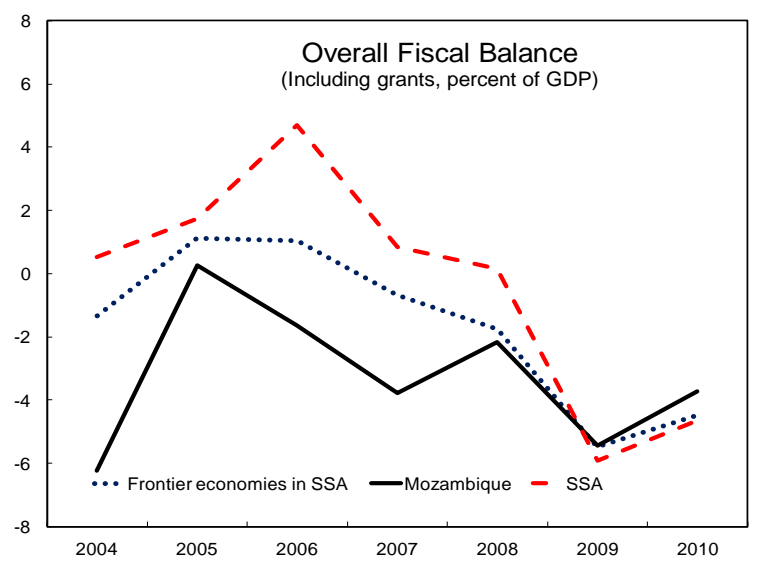

Mozambique's current account deficit was less affected by the global crisis than that of its peers.

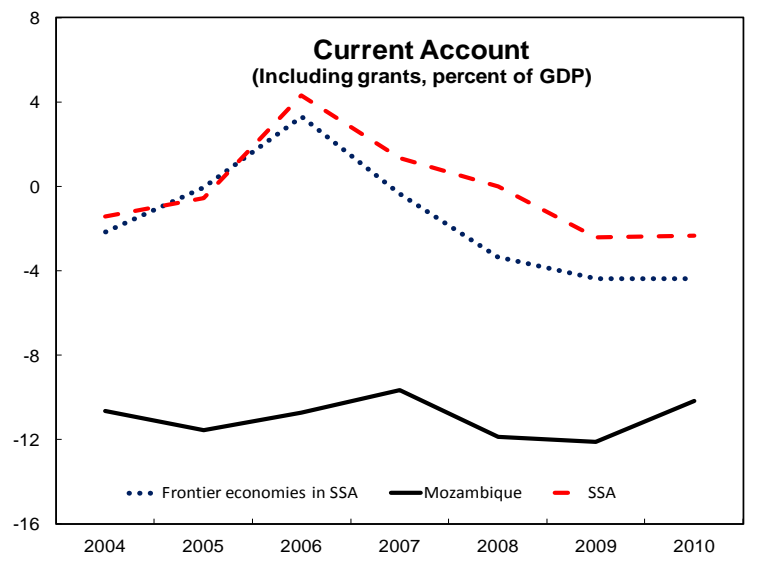

However, inflation surged above the regional levels lately.

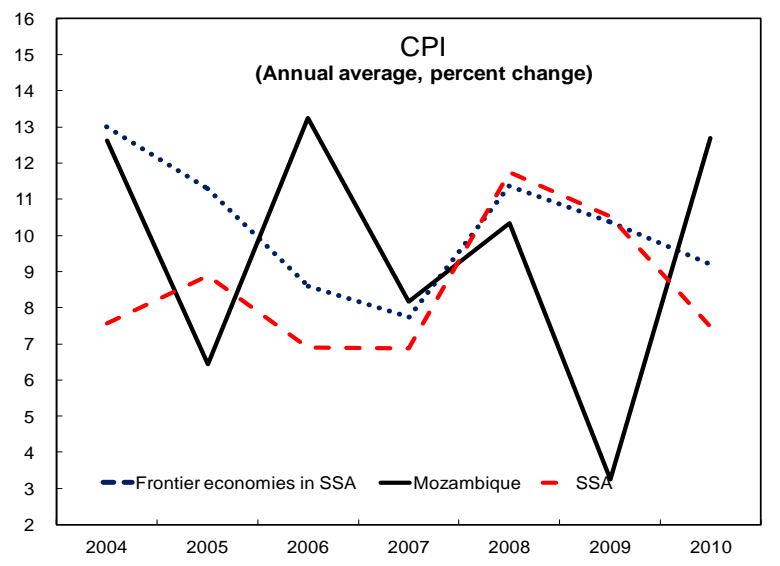

Credit expansion is slowing down as the result of the recent monetary tightening, though less than in its SSA peers.

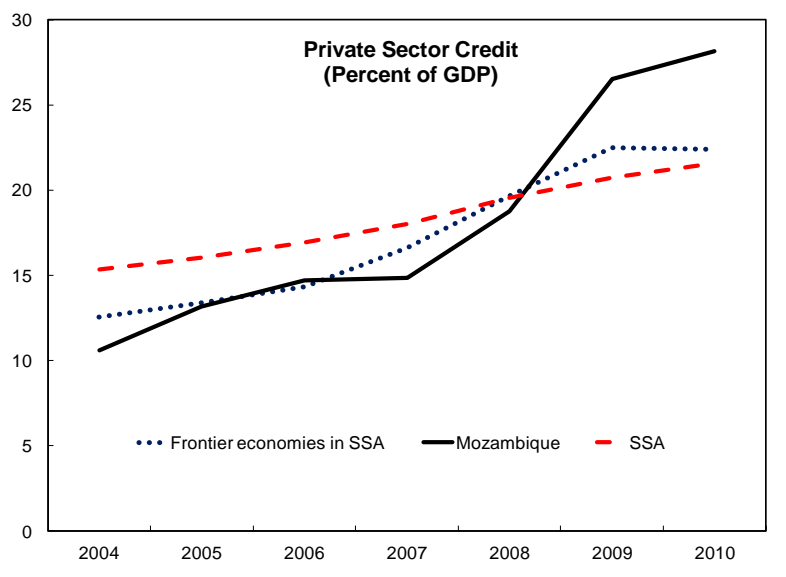

Reserves remained at more comfortable levels than elsewhere in SSA.

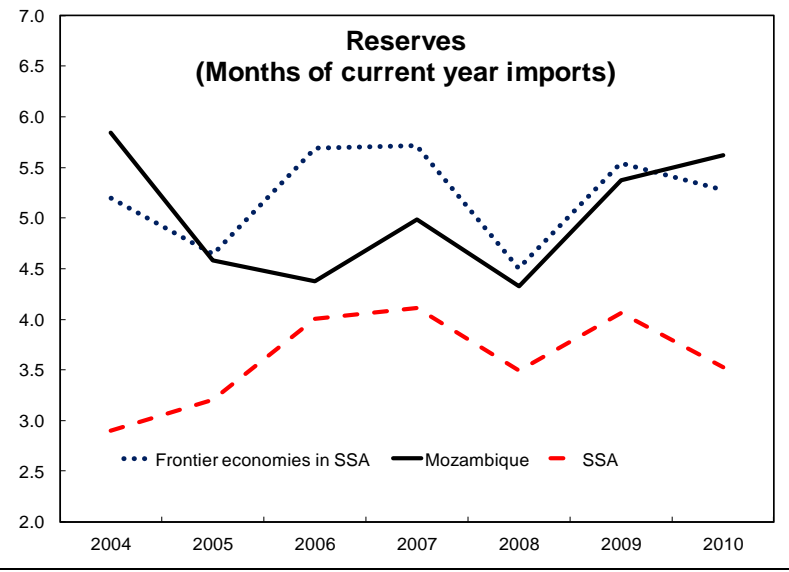

Sources: Mozambican authorities and IMF staff estimates and projections.

Frontier economies: Angola, Ghana, Kenya, Mauritius, Mozambique, Nigeria, Senegal, Tanzania, Uganda and Zambia. 
3. Mozambique's balance of payments strengthened in 2010. The current account deficit narrowed more than expected. Notwithstanding weakening traditional exports, buoyant mega-projects exports and a smaller-than-expected import bill put the current account deficit (after grants) at 101/2 percent of GDP. This, together with sustained net private capital inflows, resulted in a comfortable import cover of reserves of close to 5 months.

\section{Since mid-2010, the BM has} tightened monetary policy significantly to counter inflation expectations. It has raised its key lending rate and reserve requirements in several steps by 5 and 1 percentage points, respectively. ${ }^{1}$ It has also absorbed liquidity through sales of T-bills and foreign exchange. As a result, the expansion of monetary aggregates and private sector credit slowed down
NCG Weekly Flows at the Central Bank, 2008-2010

\begin{tabular}{lrrr}
\hline & Dec-08 & \multicolumn{1}{c}{ Dec-09 } & \multicolumn{1}{c}{ Dec-10 } \\
\hline & \multicolumn{3}{c}{ (MT Millions) } \\
1st week & 668.7 & -57.7 & $-1,063.0$ \\
2nd week & $-2,279.9$ & -105.2 & 150.6 \\
3rd week & $1,192.5$ & $-1,897.9$ & $1,127.7$ \\
4th week & -460.5 & 108.3 & $1,363.9$ \\
5th week & -298.0 & -480.2 & $-4,699.5$ \\
\hline
\end{tabular}

Source: Mozambican authorities and IMF staff estimates

markedly. Nonetheless, these policy actions were somewhat thwarted by unusually high government spending in the third and fourth weeks of December. This lack of coordination between the government and BM made it impossible for the BM to meet the end-year reserve money program target for lack of time to sterilize this liquidity injection.

\section{Remarkably strong revenue collections and spending restraint helped contain} the fiscal deficit in 2010. Both the primary and overall fiscal deficits were 1 percent of GDP or more below expectations and the prior year outcome, allowing the authorities to support the BM's policy stance and comfortably meet the AC on net credit to the government (NCG). Revenues surged by $2^{1 / 4}$ percent of GDP relative to 2009 , primarily thanks to a very strong tax administration effort, reflecting the authorities' excellent track record in implementing technical assistance (TA) advice. This helped offset a shortfall in donor resources, as the appreciating exchange rate in the second half of the year lowered the local currency value of foreign assistance and the late adoption of the annual budget law due to elections delayed implementation of investment projects. However, the fuel subsidy (11/2 percent of GDP) partially crowded out priority spending.

\section{OUTLOOK AND RISKS}

6. The outlook for 2011 and beyond remains favorable. Real GDP growth is projected to accelerate from 71/4 percent in 2011 to near 8 percent over the medium-term on account of new mega-project

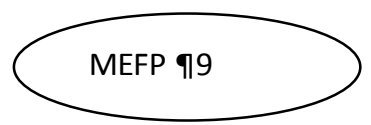
activity and stepped-up public investment, bringing Mozambique back toward its pre-crisis growth trajectory. Reflecting the authorities' policy priority to rein in inflation, the CPI is

\footnotetext{
${ }^{1}$ Given banks' low excess reserves (equivalent to $1 / 2$ percent of average reserve money in the second half of 2010), the tighter reserve requirement ratio significantly affected banks' liquidity and triggered a sharp slowdown in credit growth. However, it also contributed 31/2 percentage points to the 29 percent reserve money growth rate in 2010.
} 
projected to decline to single digits by end-2011 and reach the authorities' policy objective of 5 to 6 percent over the next two years. The current account deficit is expected to hover at 10 to 11 percent of GDP, as a rising import bill, including for food and fuel products, should be broadly offset by strong exports, including from new coal mining mega-projects, allowing the BM to build a cushion of international reserves covering 5 months of imports by 2016 .

\begin{tabular}{|c|c|c|c|c|c|c|c|c|c|c|}
\hline & \multirow{2}{*}{$\begin{array}{l}2009 \\
\text { Act. }\end{array}$} & \multicolumn{2}{|l|}{2010} & \multicolumn{2}{|l|}{2011} & \multirow{2}{*}{2012} & \multirow[t]{2}{*}{2013} & \multirow{2}{*}{$\begin{array}{l}2014 \\
\text { Proj. } \\
\end{array}$} & \multirow[t]{2}{*}{2015} & \multirow[t]{2}{*}{2016} \\
\hline & & EBS/10/210 & Est. & EBS $/ 10 / 210$ & Proj. & & & & & \\
\hline & \multicolumn{10}{|c|}{ (Percent of GDP, unless otherwise stated) } \\
\hline Real GDP growth (percent change) & 6.3 & 7.2 & 6.6 & 7.5 & 7.2 & 7.5 & 7.9 & 7.8 & 7.8 & 7.8 \\
\hline Consumer price index (annual average) & 3.3 & 12.7 & 12.7 & 8.0 & 9.5 & 7.2 & 5.6 & 5.6 & 5.6 & 5.6 \\
\hline Consumer price index (end of period) & 4.2 & 15.5 & 16.6 & 6.9 & 8.4 & 5.6 & 5.6 & 5.6 & 5.6 & 5.6 \\
\hline Credit to the economy (percent change) & 58.6 & 36.0 & 27.5 & 22.6 & 19.5 & 20.0 & 20.9 & 21.0 & 22.1 & 22.5 \\
\hline Credit to the economy & 26.5 & 29.8 & 28.2 & 31.5 & 28.6 & 29.7 & 31.4 & 33.3 & 35.6 & 38.3 \\
\hline External current account, after grants & -12.1 & -13.4 & -10.2 & -12.0 & -11.4 & -10.9 & -10.9 & -10.7 & -10.6 & -10.5 \\
\hline Overall fiscal balance, after grants & -5.4 & -4.9 & -3.7 & -7.0 & -6.4 & -6.8 & -6.4 & -5.9 & -5.4 & -5.3 \\
\hline Domestic primary balance, before grants & -4.3 & -4.1 & -3.2 & -3.3 & -3.1 & -3.4 & -3.2 & -2.8 & -2.6 & -2.5 \\
\hline Fiscal External Financing & 5.0 & 4.5 & 4.3 & 6.8 & 5.8 & 5.8 & 5.5 & 4.9 & 4.6 & 4.5 \\
\hline Domestic net credit to the governement & 0.2 & 0.3 & -0.5 & 0.2 & 0.5 & 1.0 & 0.9 & 0.9 & 0.8 & 0.8 \\
\hline Reserve Money (percent change) & 27.3 & 25.0 & 29.2 & 13.0 & 14.3 & 16.9 & 18.7 & 22.0 & 22.2 & 22.8 \\
\hline Reserves (months of projected imports) & 5.4 & 4.4 & 4.8 & 4.5 & 4.5 & 4.7 & 4.7 & 4.8 & 4.9 & 5.0 \\
\hline
\end{tabular}

Sources: Mozambican authorities; and IMF staff estimates and projections.

\section{There are several risks to the economic outlook in the short run:}

- Inflation expectations may have become too entrenched already, which may lengthen the time that the authorities need to maintain a tight macroeconomic policy stance, with adverse implications on private sector activity and bank soundness and profitability.

- $\quad$ Continued high spillovers of international food and fuel prices may negatively affect social stability, given the absence of significant safety nets; the September 2010 street riots were triggered by rising food prices. Such spillovers may be exacerbated by adverse exchange rate movements, such as from an appreciating rand as a result of continued strong capital inflows to South Africa; the large neighbor accounts for more than one-third of Mozambique's total trade and most imports of food products.

- While staff projections on the coming online of new mega-projects are prudent, the projects' smooth exports hinge on the authorities and investors rapidly addressing bottlenecks in transportation infrastructure.

8. Going forward, the largest risks to economic and social development would come from continued insufficient growth dissemination and a slowdown in donor support. As outlined below, the authorities aim to create more inclusive growth over the medium term. This partially hinges on enhancing the contribution of mega-projects to government revenue and safeguarding

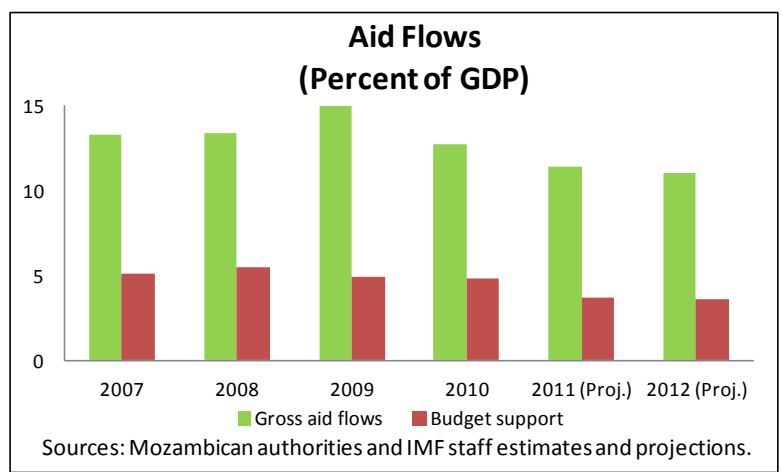


donor support to help provide sufficient resources for addressing the country's development needs in a sustainable way. While donor support has long been expected to gradually decline after its peak during the global crisis, any abrupt withdrawal, possibly as a result of budget problems in donor countries or flared-up governance concerns (see Box 1 in Country Report No. 10/174), could likely not be offset by a parallel tapping of new revenues from the natural resource sector. As a result, the authorities' envisaged ambitious reduction in poverty may not materialize within the envisaged time frame.

\section{ShORT-Term Challenge: Fighting INFLATion}

9. The authorities were adamant that tackling inflation was an immediate priority. They recognized that to achieve single-digit inflation, continued monetary tightening was needed until non-food and non-fuel inflation was brought down and inflation expectations were prevented from becoming further MEFP $\ 10-13$ entrenched. To that end, they considered it preferable to accelerate the tightening in the short run and to adopt a more accommodating policy stance once core inflation had clearly abated (Figure 4). While they saw the cost of a temporary slowdown of credit expansion which could stifle private sector activity, they on balance expected a front-loaded and decisive response to be more promising in curbing inflation expectations - a view that was shared by the mission. In light of this, the BM emphasized its determination to strictly control reserve money growth to about 14 percent in 2011, making full use of its available set of monetary instruments. ${ }^{2}$ In addition, the March 2011 agreement on information sharing between the Minister of Finance and the BM should considerably facilitate the predictability of budget execution and the forecasting of liquidity in the banking system, thus supporting the intended policy stance and helping avoid a repeat of the end-2010 lumpiness of spending that complicated the BM's conduct of monetary policy.

\section{In its monetary policy implementation, the $\mathrm{BM}$ agreed that the exchange rate} should adjust freely but emphasized that exogenous factors could complicate the policy stance. Staff analysis suggests that the current value of the real effective exchange rate is broadly in line with fundamentals, following the depreciation of the metical during 2010 (see Appendix II).

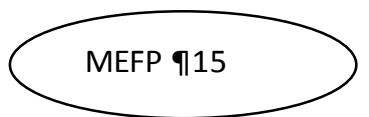

The BM shared this analysis and stressed that it would continue to pay close attention to the effective exchange rate vis-à-vis a broad basket of currencies, rather than focusing on bilateral exchange rates. Nonetheless, it expressed concern that exchange rate volatility in 2010 had partially contributed to headline inflation and that other factors beyond its control,

\footnotetext{
${ }^{2}$ While not an issue at present, the authorities nonetheless reiterated their objective of adopting an inflation targeting (IT) framework over the medium term, supported by Fund TA. They acknowledged that further conceptual work was needed to specify the targeted inflation rate, enhance its monitoring, understand the monetary transmission mechanism, and strengthen monetary policy instruments so as to be able to steer monetary conditions effectively. The mission emphasized the importance of a persuasive communication strategy with the private sector prior to effectively adopting an IT framework.
} 
Figure 4. Mozambique: Monetary and Financial Sector Developments

While money aggregates grew slower than expected, reserve money growth was faster than programmed,...

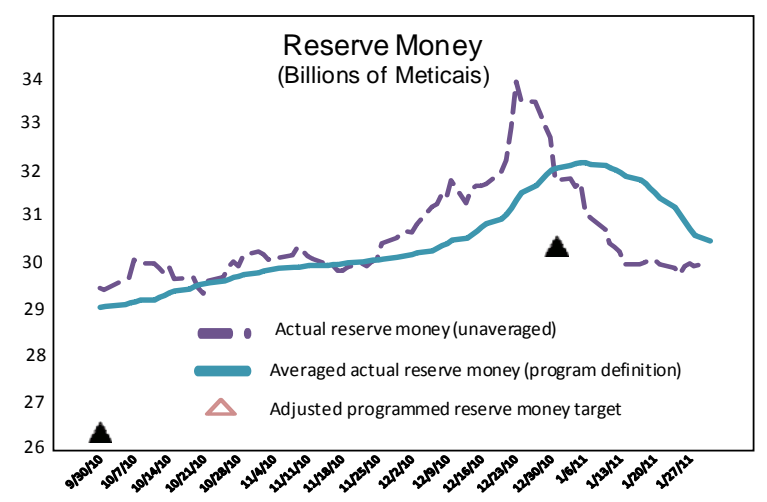

The BM has begun reversing its previous easing of monetary conditions, by decisively raising its policy rates and reserve requirements...

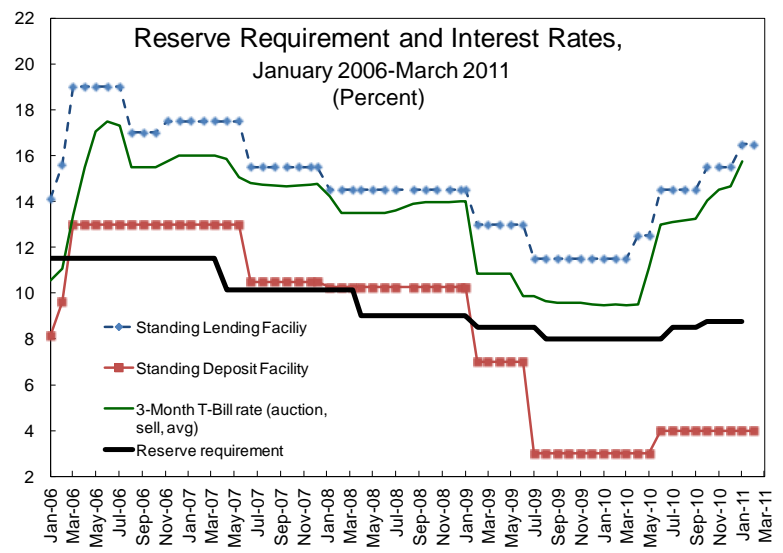

As a result, the declining trend in banks' liquidity ratios which was caused by rapid credit expansion during the monetary easing has reverted recently.

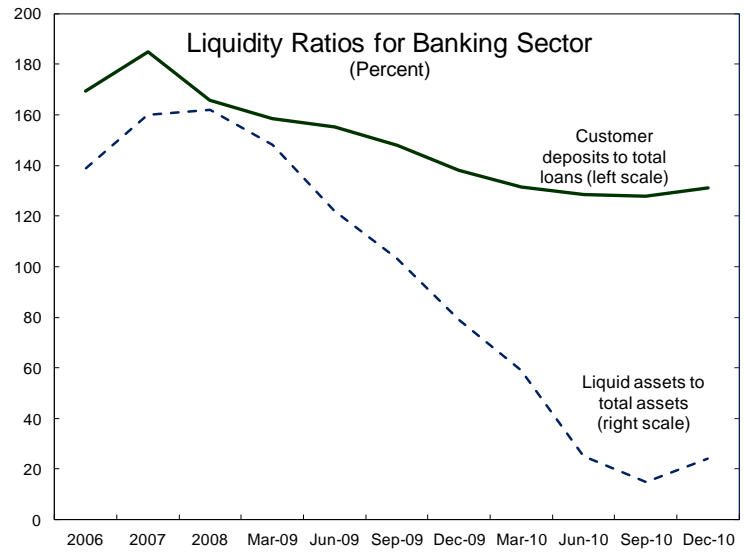

...in part due to strong government spending late in the year-to some extent undertaken in cash in un-banked regions- which the $B M$ could not sterilize in time.

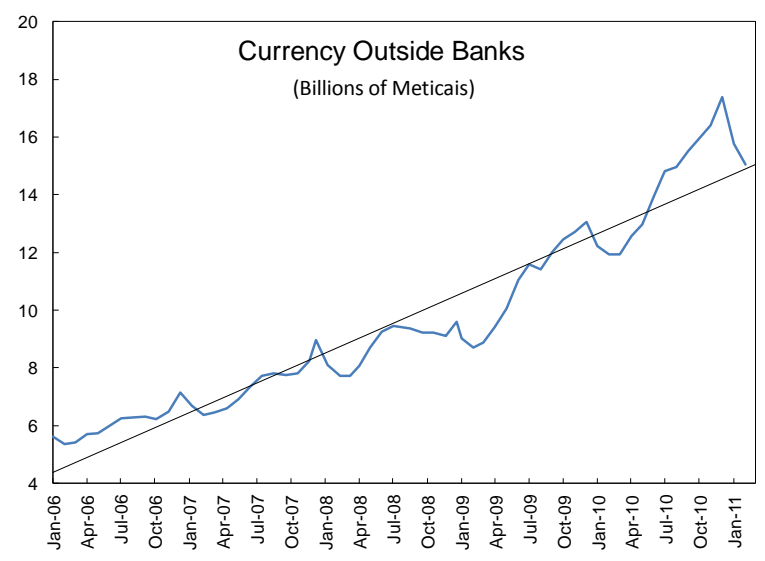

... and using its open market operations to mop up liquidity.

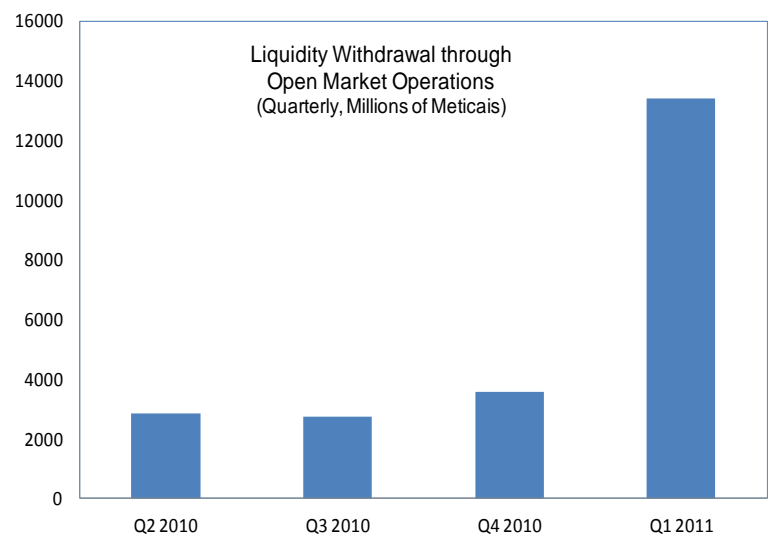

Private sector credit is expected to ease as a share of deposits, as monetary policy tightens and government borrowing rises, but will still increase relative to GDP.

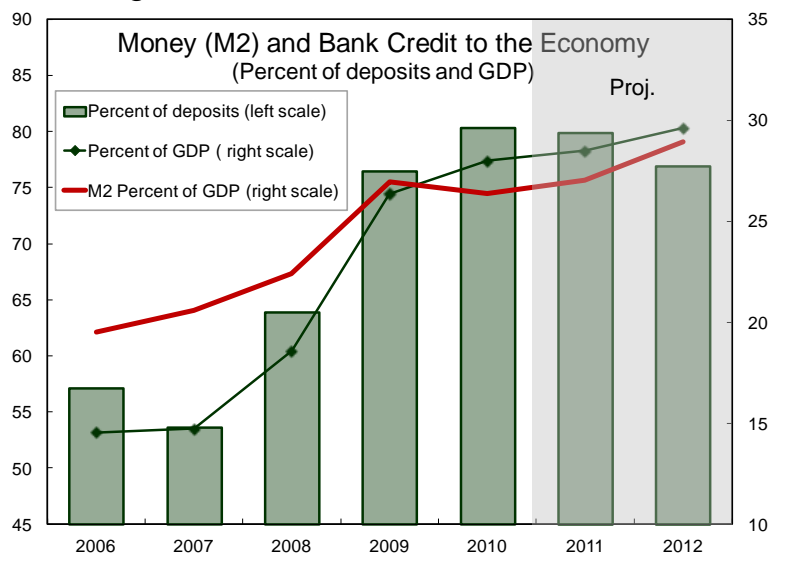

Sources: Mozambican authorities and IMF staff estimates and projections. 
such as capital inflows to neighboring South Africa, could affect bilateral exchange rates and complicate efforts to rein in inflation. Based on last year's experience, the BM intended to step up its smoothing of undue fluctuations in the relatively shallow foreign exchange market going forward, yet without managing the exchange rate. It also expressed concern that a further increase in international oil prices could undermine its efforts to safeguard NIR in light of a sharply rising import bill.

\section{The authorities agreed that while monetary policy should take the lead, fiscal} policy should be supportive of disinflation as well (Figure 5). To this end, the government has decided to tighten fiscal policy relative to earlier intentions for 2011. As a result, the overall fiscal deficit, at about $6 \frac{1}{2}$ percent of GDP, would be $1 / 2$ percentage point lower than previously envisaged.

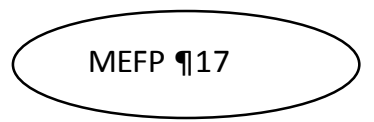
While the deficit would still widen compared with 2010, reflecting mainly the boost in public infrastructure investment, the authorities felt that given the high import content of this investment, the impact on domestic demand would be limited. Moreover, the government intends to limit its recourse to domestic financing to $1 / 2$ percent of GDP, thereby preventing a crowding out of the private sector. The mission agreed that a tightening of fiscal policy

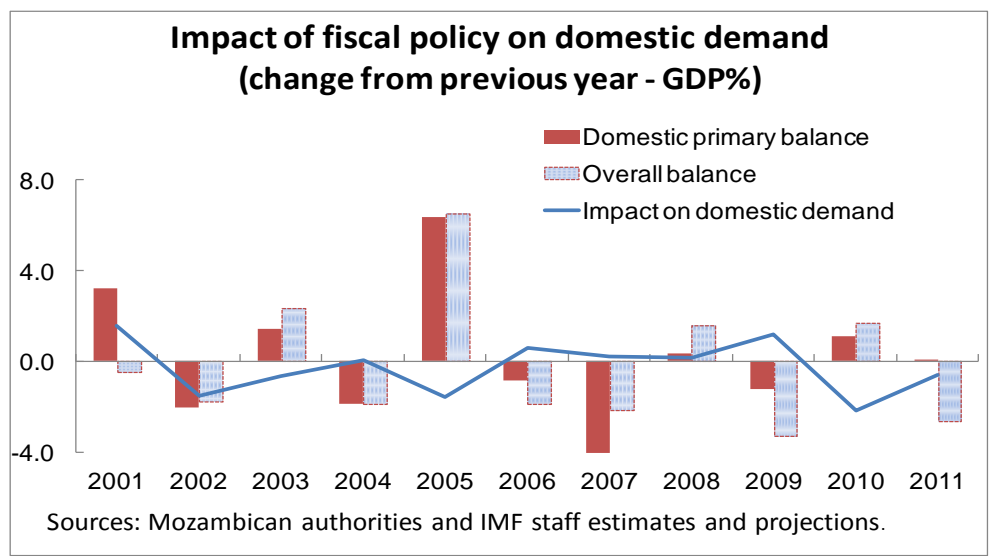
would support the disinflation effort and urged the authorities to go further in this regard, including by applying part of any revenue overperformance to further reduce the government's recourse to domestic financing.

\section{In late April, the government submitted a revised budget law to Parliament.}

While curtailing the overall spending envelope, it envisages a reallocation from capital to current spending. It also takes into account the lower domestic currency value of donor support in light of the appreciated exchange rate, which necessitates a slightly higher NCG. The reallocations became MEFP $\rrbracket 18$ necessary to afford the costs of the fuel subsidy - including related to 2010, based on an agreement signed with fuel importers in January 2011 — and provide resources for the new emergency measures to preserve social peace (see below). Recognizing the significant burden on the budget, the government in late March announced the full yet gradual removal of the fuel subsidy; fuel prices were raised in April for the first time in six months. 
Figure 5. Mozambique: Fiscal Developments

Revenue collections in 2010 were buoyant, reflecting tax administration efforts.

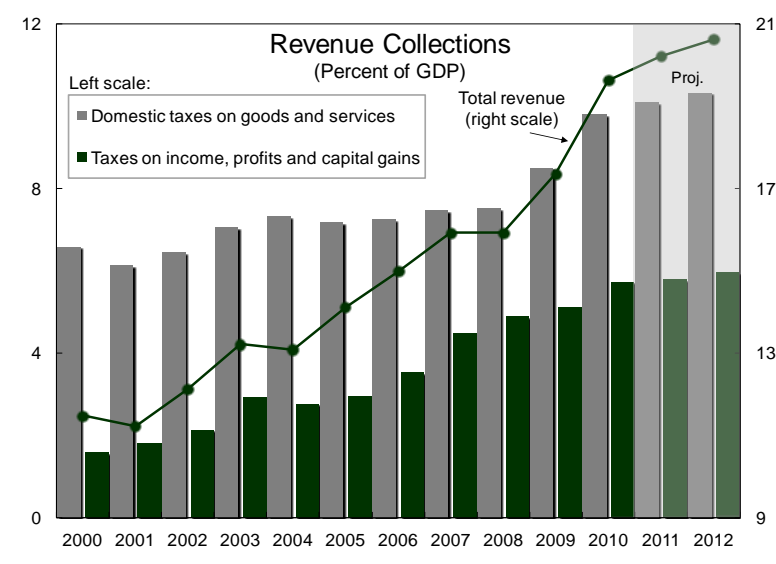

The domestic primary deficit was contained in 2010.

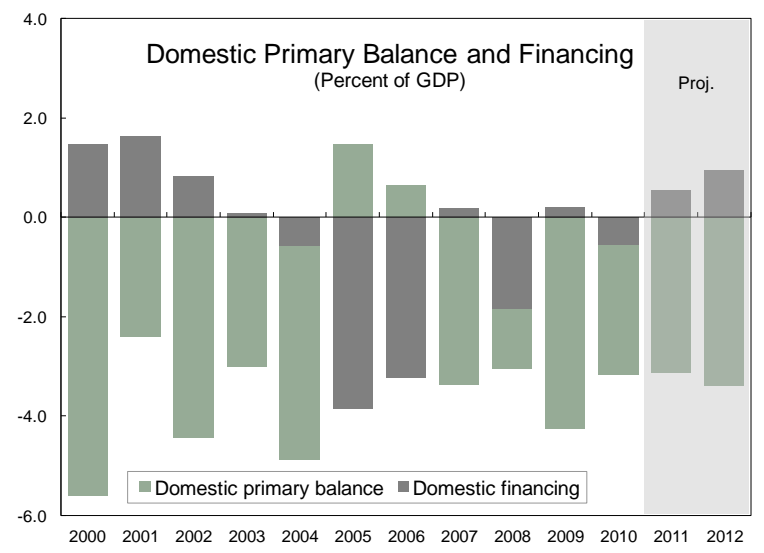

...external budget support which so far has helped reduce the need for domestic financing.

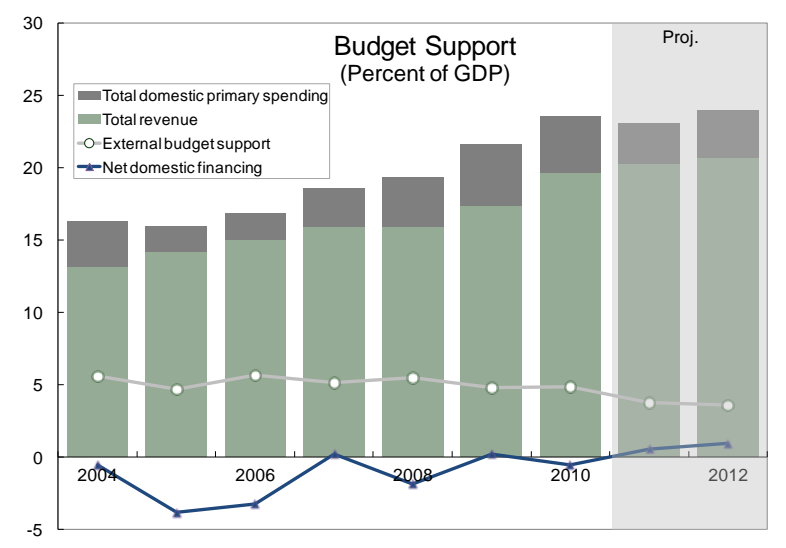

The wage bill and transfers-especially for the fuel subsidy_put pressures on domestic spending.

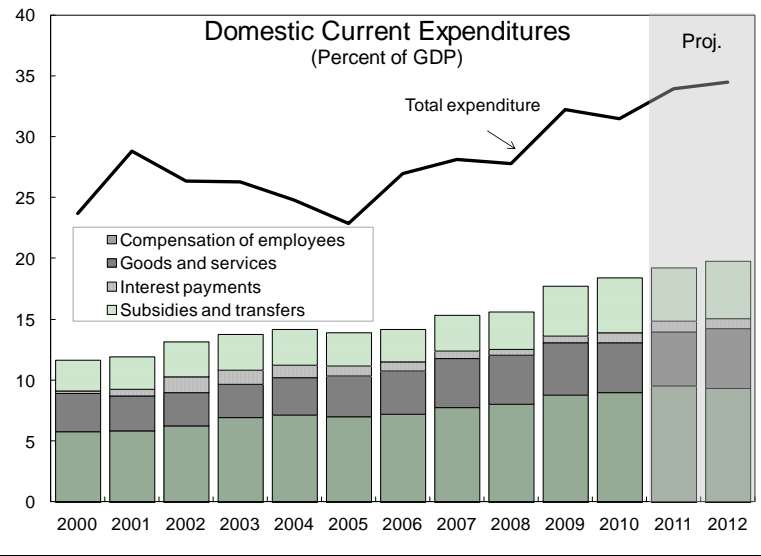

Capital spending relies heavily on external project support which has been on a declining trend, and...

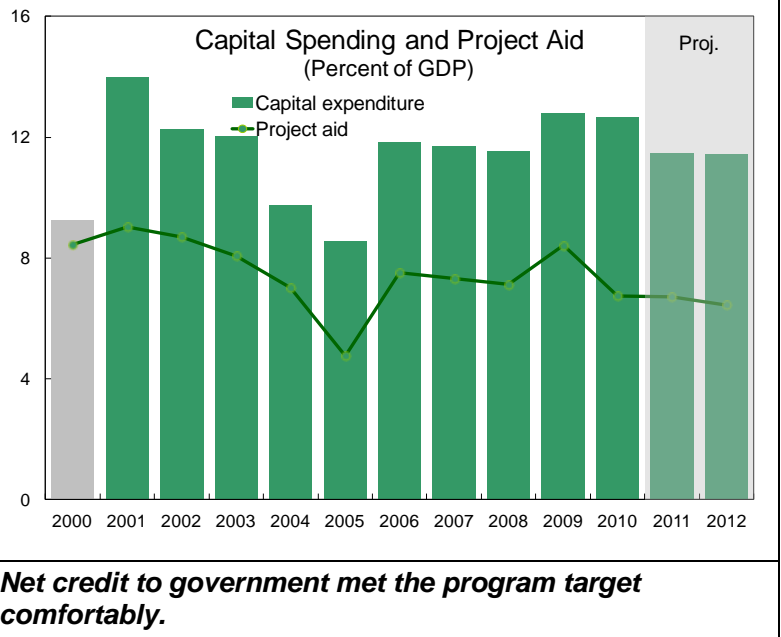
comfortably.

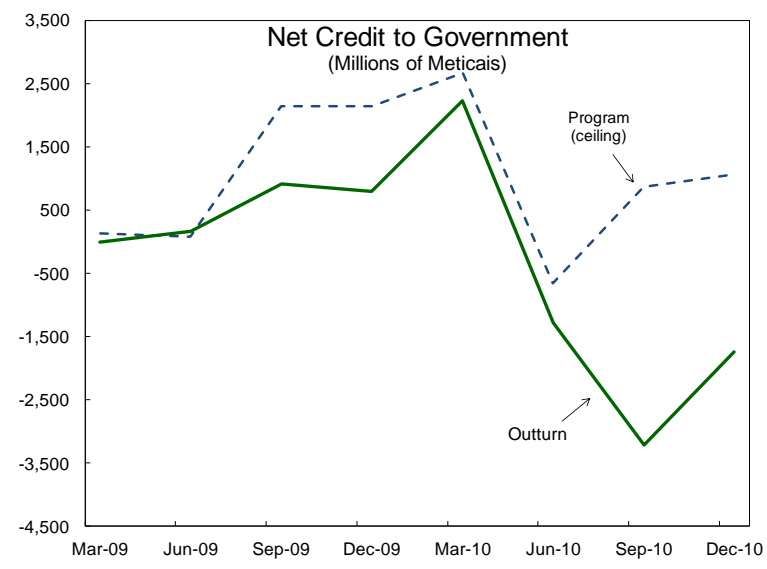

Sources: Mozambican authorities and IMF staff estimates and projections. 


\section{Medium-Term Challenge: BUILding STABLE AND INCLUSIVE GROWTH}

\section{A. Creating Fiscal Space to Sustain Investment and Support Broad-Based Growth}

13. The authorities' medium-term growth model to create the conditions for sustainably raising economic growth and reducing poverty is built on significantly stepping up public investment. To meet the country's development needs and close the skills and infrastructure gaps, fiscal policy will aim at creating fiscal space through a sustained revenue effort and increasing recourse to domestic and external borrowing, including on nonconcessional terms (a cumulative US\$900 million over the period of the PSI). The new sources of fiscal space are expected to also offset the gradual decline in donor resources from their peak during the global crisis.

\section{A Prudent Start of Nonconcessional Borrowing}

14. The authorities reiterated that their investment strategy would be consistent with macroeconomic stability and debt sustainability. They were particularly mindful of the need to avoid any undue domestic demand

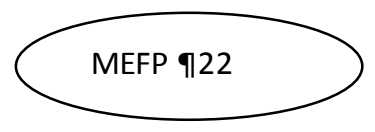
pressures in the current inflationary environment. Their decision to pursue a very prudent approach in negotiating any nonconcessional borrowing (NCB) with potential partners will help stretch somewhat the public investment profile compared to earlier projections, without scaling back the overall multi-year investment envelope. Reflecting the revised public investment and borrowing plans, a DSA update suggests that Mozambique continues to face a low risk of debt distress (see Supplement 1).

\begin{tabular}{|c|c|c|c|c|c|c|c|}
\hline & 2009 & 2010 & 2011 & 2012 & 2013 & 2014 & 2015 \\
\hline & \multicolumn{7}{|c|}{ (Percent of GDP) } \\
\hline Total & 12.8 & 14.0 & 15.6 & 15.2 & 15.1 & 14.6 & 13.9 \\
\hline On budget & 12.8 & 12.6 & 13.7 & 13.8 & 14.0 & 13.7 & 13.3 \\
\hline Domestically financed & 4.4 & 5.9 & 4.9 & 5.0 & 5.2 & 5.3 & 5.2 \\
\hline Donor financed, concessional & 8.4 & 6.7 & 6.7 & 6.4 & 6.6 & 6.6 & 6.6 \\
\hline Non-concessional & 0.0 & 0.0 & 2.1 & 2.4 & 2.2 & 1.8 & 1.6 \\
\hline Credit line from Portugal 1/ & 0.0 & 1.3 & 1.9 & 1.4 & 1.1 & 0.8 & 0.5 \\
\hline Concessional & 0.0 & 1.3 & 0.9 & 0.8 & 0.5 & 0.3 & 0.3 \\
\hline Nonconcessional & 0.0 & 0.0 & 1.0 & 0.6 & 0.5 & 0.5 & 0.2 \\
\hline \multicolumn{8}{|l|}{ Memorandum items: } \\
\hline Total concessional and domestic & 12.8 & 14.0 & 12.6 & 12.2 & 12.4 & 12.3 & 12.0 \\
\hline Total nonconcessional & 0.0 & 0.0 & 3.1 & 3.0 & 2.7 & 2.3 & 1.8 \\
\hline
\end{tabular}

Source: IMF staff calculations.

1/ Portuguese credit line that will be implemented by the Road Fund.

15. The mission welcomed the authorities' prudence in tapping nonconcessional resources and the progress made in stepping up debt management and investment planning. To date, the authorities have signed two nonconcessional loans to finance infrastructure investment, using up only some oneMEFP $\mid 25$ 
sixth of the overall NCB envelope under the program: (i) in December 2010, a loan from China of US\$66 million to modernize Maputo airport; ${ }^{3}$ and (ii) in April 2011, a loan from Brazil on the construction of Nacala airport over US\$80 million. In addition, they are making good progress in modernizing their PFM, debt management, and project selection

procedures, in line with program commitments. ${ }^{4}$ All related structural conditionality was met, although the technically challenging elaboration of a medium-term debt strategy (MTDS) encountered a five-month delay. The mission and the authorities agreed to revisit the MTDS later this year once other enhancements in debt management and project selection are being fully applied and additional TA has been received.

\section{A Need to Sustain the Revenue Effort}

\section{The mission commended the authorities for the impressive increase in revenue} collections over the past five years but called for revenue diversification. Since 2005 , revenues have risen by more than $6 \frac{1}{2}$ percent of GDP, thanks to a determined tax administration effort. However, staff projections suggest that the pace of further revenue increase would slow down going forward unless MEFP $\rrbracket 26-27$ other revenue sources could be tapped. The authorities agreed with this assessment. They reiterated their intention to continue implementing their tax policy and administration reforms consistent with Fund TA recommendations, which should allow Mozambique to approach the average collections of peer countries in the SADC region.

\section{The authorities indicated that they would consider options to enhance revenues}

from the natural resource sector. Compared to other resource-rich countries, minerals revenues account for only a fraction of total revenues in Mozambique, reflecting both the nascent production in the natural resource sector and special tax regimes and exemptions granted to foreign investors before a new fiscal regime to tighten the tax treatment was established in 2007. ${ }^{5}$ Given the country's major development needs, a vibrant public

\begin{tabular}{lcc}
\multicolumn{3}{c}{$\begin{array}{c}\text { Hydrocarbon and Mineral Revenues } \\
\text { (Average 2000-2007) }\end{array}$} \\
\hline \multicolumn{1}{c}{ In percent of GDP } & $\begin{array}{c}\text { In percent of } \\
\text { Total Revenue }\end{array}$ \\
\hline Hydrocarbons and Minerals-Rich Countries & 17.3 & 43.0 \\
Hydrocarbons-Rich Countries & 20.0 & 48.9 \\
Minerals-Rich Countries & 12.8 & 37.8 \\
$\begin{array}{l}\text { Memorandum item: } \\
\text { Mozambique (Average 2008-2010) }\end{array}$ & 1.8 \\
\hline Source: Mozambican authorities and IMF staff estimates.
\end{tabular}

\footnotetext{
${ }^{3}$ The loan, denominated in RMB, has an estimated grant element of 31 percent, marginally below the minimum concessionality of 35 percent. Staff's calculations reflect using the SDR-CIRR discount rate (as is usually the case with loans denominated in the currency of other non-OECD countries). The authorities and China are considering renegotiating the loan to make it fully concessional.

${ }^{4}$ A new PEFA assessment was released in December 2010, confirming continued strong progress in enhancing the PFM framework. The new assessment and the strong sub-CPIA rating could have a positive impact in the next capacity assessment exercise under the Fund's debt limits policy.

${ }^{5}$ Staff estimates that total taxes paid by companies in the mega-project and mining sector account for about 5 percent of their profits. If the 2007 fiscal regime was fully applied, companies in this sector would be subject to royalty and income taxes and expected to pay an average rate of at least 30 percent on their profits.
} 
debate has emerged in Mozambique on renegotiating existing contracts. The mission recommended that the authorities adopt a prudent approach in this regard that balances the interest to generate additional revenues for the budget with protecting Mozambique as a destination for foreign investment. It pointed to experience from elsewhere (e.g., Peru, Tanzania) which suggested

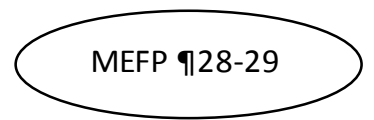
aiming for an amicable agreement with existing investors by trying to convince them that social peace and economic development in the country were in their interest as well. Should such efforts be fruitful, the authorities would consider the creation of a stabilization fund to smooth inter-temporal natural resource revenue flows and pro-growth spending over the long term. In the meantime, Mozambique has taken all necessary steps to soon become a fullfledged member under the Extractive Industries Transparency Initiative (EITI).

\section{B. Building Inclusive Growth}

\section{Stagnant Poverty Reduction}

18. Mozambique's growth take-off has not been accompanied by economic diversification nor by commensurate employment creation. Its productive and export base has become increasingly concentrated - a reflection of the emergence of mega-projects and weaknesses in the business environment that have prevented diversification of the economy, in contrast with SSA peer countries.

\section{The September 2010 riots prompted the authorities to rethink their economic} growth strategy (see Country Report No. 10/375, Box 2). The unrest underlined the extreme vulnerability of the urban poor to a price shock and the lack of social safety nets.

Furthermore, the third national household survey (2008/09) published in 2010 indicated that the incidence of poverty had stagnated at 55 percent since 2003, after a decline from 69 percent in 1997.

\section{Staff analysis confirms that} growth in Mozambique has not been as pro-poor as in other highgrowth countries and become less pro-poor over time (Appendix III). The elasticity of the headline poverty rate with respect to GDP growth is much lower than in peer countries. Staff calculations of a growth incidence curve suggest that households in the lowest three deciles experienced negative expenditure-per-capita growth, while others benefited from positive

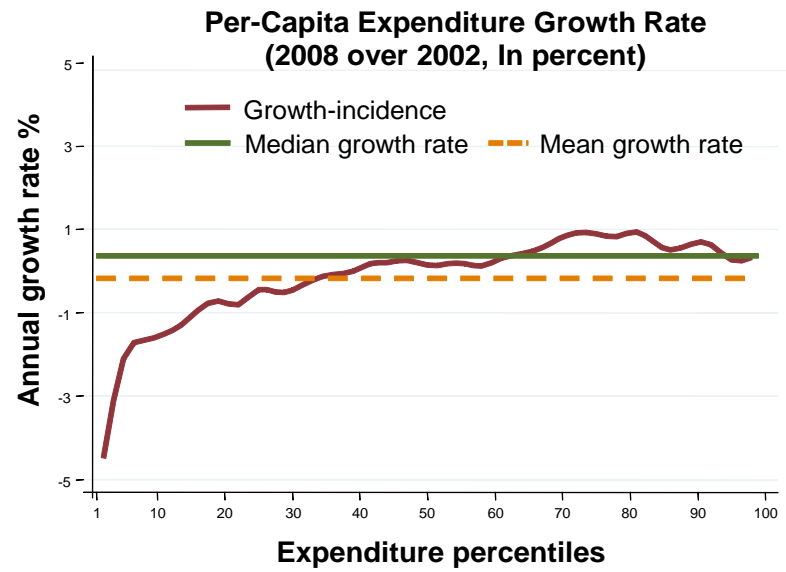

Sources: Mozambican authorities and IMF staff estimates. growth. 


\section{In spite of the lack of poverty reduction, Mozambique's performance with} respect to the MDGs shows notable improvements. This holds particularly true for primary education and basic health, although the weak link between growth and employment becomes evident as well.

\begin{tabular}{lrrrrr}
\multicolumn{5}{c}{ Selected Millennium Development Goals, 1990-2009 } \\
\hline & 1990 & 1995 & 2000 & 2005 & 2009 \\
\hline Goal 1: Eradicate extreme poverty and hunger & & & & & \\
Employment to population ratio, 15+, total (\%) & 80 & 79 & 79 & 78 & 78 \\
Employment to population ratio, ages 15-24, total (\%) & 67 & 68 & 67 & 66 & 66 \\
Goal 2: Achieve universal primary education & & & & & \\
Primary completion rate, total (\% of relevant age group) & 26 & 26 & 16 & 42 & 57 \\
Total enrollment, primary (\% net) & 44 & 44 & 56 & 76 & 91 \\
Goal 4: Reduce child mortality & & & & & \\
Mortality rate, infant (per 1,000 live births) & 155 & 139 & 123 & 109 & 96 \\
Mortality rate, under-5 (per 1,000) & 232 & 207 & 183 & 162 & 142 \\
Goal 5: Improve maternal health & & & & & \\
Maternal mortality ratio (modeled estimate, per 100,000 live births) & 1,000 & 890 & 780 & 640 & 550 \\
Pregnant women receiving prenatal care (\%) &.. & 71 & 76 & 85 & 89 \\
\hline
\end{tabular}

Source: World Development Indicators. Figures in italics refer to periods other than those specified.

\section{A Strategy for Inclusive Growth}

\section{Against this background, the authorities intend to broaden the country's} development strategy to make economic growth more inclusive. A high-level conference in Indy Village (Maputo) in February 2011 provided a forum to discuss ways for Mozambique to achieve this objective (Box 2).

\section{Box 2. Indy Village Seminar on Inclusive Growth}

The three-day high-level conference took place in the aftermath of the September 2010 urban riots and recent data evidence of stagnant poverty reduction. It was designed to allow the Mozambican authorities to learn from international experience on how to adjust their growth model and design the new poverty reduction strategy. It was organized by the authorities, in close coordination with the World Bank, the Fund, the African Development Bank, and UK-DfID, followed the blueprint of the successful 2010 Namaacha conference, ${ }^{1}$ and attracted some 100 participants from the authorities, civil society, private sector, donors, and academia.

The discussions concluded that economic growth in Mozambique would need to be sustained over several more years, and be broader and more diverse, to become inclusive. Based on the analysis of cross-country experiences, including from China and Brazil, this would need to entail a structural transformation of the economy to allow the emergence of a more competitive and diversified productive and export base and boost production and productivity in labor-intensive sectors, with a special focus on agriculture. Participants also stressed the need to preserve Mozambique's key assets, such as the strong track record of macroeconomic stability, and agreed with the authorities' emphasis to boost public investment as a way to crowd in private sector activity. It was also suggested to further improve the management of revenues from the country's natural resources to support closing the infrastructure and skills gaps and expand social protection.

The conclusions were presented by Bank and Fund staff, and discussed, in a ministerial meeting chaired by the Prime Minister. The conference received wide media coverage.

${ }^{1}$ See Box 3 in Country Report No. 10/174. 


\section{The new Poverty Reduction Strategy (PARP) for 2011-14 was adopted in early}

May 2011. It leaned heavily on the Indy Village seminar. Pending a more thorough joint staff assessment of the document with the World Bank in the context of the third program review, the mission welcomed the overall consistency of the PARP with the authorities' economic program under the PSI and the appropriateness of its three main pillars to take up the challenge of growth dissemination (Box 3). The mission underscored that the challenge will be in implementing the strategy in a coordinated manner with all stakeholders.

\section{Box 3. Action Plan for Reducing Poverty (PARP) 2011-14}

The PARP is a concise document that establishes the achievement of inclusive growth as one of its overarching objectives. Based on a candid assessment of the extent of poverty-including uneven progress across regions, urban and rural districts, and genders - the authorities' ambition is to reduce poverty incidence from 55 percent in 2009 to 42 percent in 2014.

The strategy is based on three interlinked main pillars that require extensive inter-ministerial coordination:

(i) Increased production and productivity for the agricultural and fisheries sectors;

(ii) Promoting employment; and

(iii) Human and social development.

The plan's three main pillars benefit from two supporting pillars focused on fostering good governance and preserving macroeconomic stability. The PARP's objectives and indicators will be reviewed, and if needed, updated annually under the Economic and Social Plan (PES) in light of developments and in tandem with the implementation of underlying sectoral strategies.

\section{Expanding Social Protection Systems}

\section{The mission advised the authorities to leverage fiscal space to design sustainable} social protection systems. The authorities acknowledged that the fuel subsidy was illtargeted and fiscally unsustainable, as outlined above, and that there was scope to replace the emergency subsidies introduced in the wake of the September 2010 unrest. To this end, they announced new basic

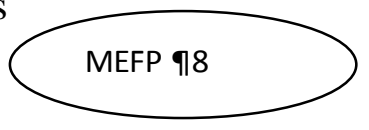
food and transport voucher programs for the urban poor, to be introduced from mid-2011 onwards. The mission, but also development partners and civil society, expressed concern about the targeting, implementation constraints, and potential administrative cost of the proposed voucher programs and recommended that the authorities seek outside expertise to refine them or develop alternative measures. In this context, the mission emphasized that the removal of the fuel subsidy created a unique opportunity to redirect public resources towards perennial and better targeted social protection schemes, which could be gradually rolled out, based on a joint costing pilot work involving various development partners, including the Fund (Box 4). It underscored that Mozambique should seize this momentum to become a "front runner" in sustainable social protection in Sub-Saharan Africa. The authorities indicated that they would deepen the dialogue with relevant stakeholders on the various options and decide around July 2011 on the future direction of basic social security. 


\section{Box 4. Social Protection in Mozambique}

Social protection policy in Mozambique has received more impetus lately. The National Strategy for Basic Social Security (ENSBB) - adopted in April 2010 and aiming at promoting an integrated approach to social protection - and the Regulation for Basic Social Security (RBBS) approved in December 2009 and organizing social protection in four components (social transfers, health, education, social inclusion through work)—were essential milestones towards establishing a national social protection floor.

Nevertheless, the coverage of the population remains limited. The cornerstone of social security is the Food Subsidy Program (PSA). Although the national coverage of this cash transfer program has more than doubled since 2006 to 220,000 beneficiaries, the amount of transfer is low. Overall, social protection programs cover only about 8 percent of poor households.

Strategic decisions to scale up social protection are expected to be taken by the Council of Ministers in July 2011. The policy dialogue is being informed by a joint pilot exercise involving the UN (including the ILO), the Bank, the Fund, and bilateral partners. The exercise includes (i) a Bank-led review of existing social security programs and expenditure; (ii) a costing exercise led by the ILO, focusing on public works programs, conditional cash transfers, and a universal pension system; (iii) a Fund-led assessment of available fiscal space consistent with the macroeconomic framework; and (iv) a simulation of the impact of policy options on the poverty gap, led by UNICEF.

\section{Fiscal Decentralization to Improve Spending Outcomes}

\section{The authorities intend to strengthen the link between spending and outcomes} through a gradual decentralization policy (Box 5). They are following a two-pronged approach: (i) a simple de-concentration of budget implementation to units of the central government posted in districts outside the capital, MEFP 930 which currently accounts for 30 percent of total spending; and (ii) a gradual devolution of selected taxation and spending responsibilities to high-capacity municipalities (e.g., Maputo, Beira), which at present represents less than $1 \frac{1}{2}$ percent of total spending. The authorities viewed their experience on both fronts positively and saw merit in further increasing local involvement going forward, as they expected service delivery to improve.

\section{The mission welcomed these intentions but pointed to the need to further} develop capacity and monitoring standards before expanding such efforts on a larger scale. Based on cross-country experience, it advocated that fiscal de-concentration, rather than devolution, would be a sensible step forward in the current environment, given that most municipalities in the vast territory do not yet have sufficient administrative capacity. As such, de-concentration could be seen as a way to build this capacity before smaller municipalities would be in a position to gradually take over authority for taxation and spending responsibilities. In this context, important questions needed to be tackled, such as on the feasibility of improving the effectiveness and transparency of existing vertical transfer systems, the need for horizontal equalization of tax revenues (since some regions 
may reap large dividends from the booming natural resource sector), and the elimination of currently overlapping responsibilities between districts and municipalities.

\section{Box 5. Fiscal Decentralization in Mozambique}

Fiscal decentralization in Mozambique aims to (i) enhance participation of the local population in the definition of spending priorities, (ii) strengthen administrative capacity at the district and municipal level, and (iii) improve the link between spending and outcomes. Early in the post-civil war period, the authorities favored devolution through municipalities, which in 2002 was complemented by a de-concentration approach in view of the lack of capacity of most municipalities in sparsely populated areas. Since some municipalities (e.g., Maputo, Beira) have enhanced their capacity to manage key assets and services, a Law on Municipal Finance was approved in January 2008 to enhance the framework of fiscal devolution. This law will be complemented by an implementation strategy, expected to be approved in mid-2011.

Municipalities both collect revenues and benefit from central government transfers. They directly collect various service fees and taxes, such as on property and vehicle registration. They also benefit from central government transfers to finance current and capital expenditures. These transfers are allocated across municipalities according to population and other criteria. On the expenditure side, municipalities are responsible for road maintenance, public transportation, solid waste collection, land management, and urban planning. Primary and secondary education and basic public health are also executed locally but centrally managed by the central government.

Districts are the centerpiece of the de-concentration policy. They are assigned to manage the local civil service, including primary school teachers, purchase goods and services for the administration, and elaborate a local investment plan in consultation with local citizens. Although districts are empowered to levy some taxes, in practice their budget relies almost exclusively on transfers from the central government.

27. The authorities broadly agreed with the mission's reasoning. Nonetheless, they intended to go ahead with the planned mid-2011 adoption of the implementation strategy for the further devolution of responsibilities to municipalities. They indicated that the strategy would take the need for adequate capacity standards into consideration. The mission stressed the importance of the strategy also clearly spelling out minimum requirements for internal and external controls and reporting mechanisms so as to minimize fiscal risks and ensure value for money. Given the complexity of the tasks at hand, the mission also encouraged the authorities to seek the expertise of development partners.

\section{Strengthening the Financial Sector}

\section{The authorities view the financial sector as an essential ingredient to sustain} growth. They are finalizing their 2011-20 Financial Sector Development Strategy which follows up on the recommendations of the 2009 Financial Sector Assessment Program (FSAP) update. It includes a wide-ranging and MEFP $¥ 33-34$ clearly sequenced action plan, covering ways to, among other things, enhance financial inclusion, competition, consumer protection, and financial literacy. The mission emphasized the key role that a more developed financial sector could play in diversifying the investor base in Mozambique and promoting long-term growth. 
29. The banking sector appears to have withstood the global crisis reasonably well, but continuous vigilance in banking supervision is needed. Against the backdrop of very strong credit growth and high foreign ownership of domestic banks, system-wide indicators suggest that profitability and liquidity, while abating over the past two years, remain at reasonable levels. The capitalization of the banking sector remains comfortably above required levels and compares well with the 2009 FSAP update. Stress tests prepared by staff and shared with the authorities, however, signaled one undercapitalized medium-small bank on which the authorities should act decisively, and a persistent high concentration risk throughout the banking sector (Appendix IV).

30. The mission cautioned that Mozambique's specific provisioning rules hindered cross-country comparison and could generate lags in the recording of non-performing loans. ${ }^{6}$ Their level had remained low and stable at about 2 percent of gross loans while claims on the private sector tripled during 2008-10. The authorities explained that they had adopted a gradual approach and first focused on aligning their loan classification rules in 2009. The mission encouraged the authorities to align the remaining provisioning rules in due course as well. It also noted that the welcome transition to the more risk-based Basel II standards by 2014 should further consolidate domestic banks' capital base.

\section{The authorities were confident that their regulatory framework limits the} contagion risk which could stem from distressed foreign parent institutions. They saw comfort in the recent decisions by foreign shareholders to increase the capital of the three largest Mozambican banks, all of them majority foreign-owned. They also quoted existing regulatory limitations on the concentration of domestic banks' deposits in parent banks, and considered that the BM's formal authorization of credit lines from parent banks provided additional safeguards against sudden withdrawals of liquidity. Staff urged the BM to scrutinize intra-group liquidity flows, in particular with Portuguese parent banks, and to seek participation in colleges of supervisors led by parent banks' supervisors.

\section{The mission welcomed the move towards completion of a banking crisis}

resolution framework. In addition to the already implemented prompt corrective action procedures and coordination with foreign supervisors (a structural benchmark for end-April 2011), the remaining modules of the plan will comprehend rules for closing and liquidating banks, managing bidding processes for private sector takeovers, temporary public ownership, and emergency liquidity assistance. As envisaged in the 2009 FSAP, the government is also making progress in establishing, and securing the financing for, a deposit insurance scheme. This set of reforms should enhance confidence in the banking sector.

\footnotetext{
${ }^{6}$ The provisioning regime differs from international standards insofar as provisions apply to past due installments of a loan only, and not to the entire loan, and the speed of the classification into doubtful loans depends on the residual maturity of a loan.
} 
33. Steps need to be taken for a more effective AML/CFT framework. While a Financial Intelligence Unit (GIFIM) is being made operational and benefitting from donor support, the amendment of the AML/CFT law, which would allow Mozambique to move toward compliance with the 40+9 Recommendations of the FATF, is still awaiting parliamentary approval. As Mozambique's first AML/CFT Mutual Evaluation by the Eastern and Southern Africa Anti-Money Laundering Group (ESAAMLG) has been considered by the ESAAMLG Plenary in April 2011, the mission urged the authorities to fully draw on the report's forthcoming final recommendations.

\section{MozambiQue's Economic Program Under the PSI}

34. The PSI's program design reflects the authorities' policy priorities, as outlined

above. Its focus areas benefited from a consultative approach, such as through the two conferences on Mozambique's economic challenges (Namaacha, March 2010) and inclusive growth (Indy Village, February 2011), and are consistent with the new PARP.

35. The program remains largely on track. The end-December $2010 \mathrm{AC}$ on reserve money was missed due to unexpectedly large government spending during the last two weeks of the year which the BM had little time to sterilize. Since then, the authorities succeeded in further decelerating reserve money growth, with a view to cutting it by half in 2011 relative to 2010 . In addition, the end-2010 indicative floor on priority spending was missed; while allocations for health and education were not affected, project spending in infrastructure, agriculture, and rural development suffered. The shortfall was largely due to a slower execution of externally-financed projects. In addition, while the domestic investment component for priority spending was fully spent, domestic current priority spending fell short of expectations, likely reflecting the higher costs of the fuel subsidy. The authorities met all structural benchmarks through end-April, although the MTDS experienced a longer (five-month) delay, as outlined above.

36. The program's focus remains broadly unchanged. Macroeconomic policies in 2011 are consistent with the objective to curb inflation, while supporting the country's development needs over the medium term, as outlined above. Structural reforms will continue to aim at building institutional capacity to shore up the authorities' policy objectives and improve economic decision-making, covering improvements in tax policy and administration, natural resource management, debt management, investment planning, financial sector stability, and good governance. Given their macroeconomic relevance, conditionality was set on measures related to the monitoring of the government wage bill, finalization of the financial sector contingency plan, the adoption of an integrated investment program, and the submission to Parliament of an ambitious package of reforms to support the fight against corruption - a key measure to safeguard growth and donor support. 
37. Some modifications to the quantitative program targets are proposed. First, as advised in Country Report No. 10/375, the 2011 NCB ceiling reflects the unused amount carried over from 2010 so as to support the authorities' development objectives. Second, the end-June 2011 AC on reserve money, NIR, and NCG were adjusted according to the authorities' short-term policy objectives and the end-2010 outcome. In particular, while the targeted annual reserve money growth rate for 2011 remains at 14.3 percent, the reserve money AC for end-June represents a tighter stance than previously envisaged, reflecting the authorities' intention to frontload the disinflation process. By contrast, the NCG path needed to be adjusted consistent with expected donor aid flows, taking into consideration the lower domestic currency value of such assistance in light of the appreciated exchange rate. And third, the end-June 2011 indicative floors on revenue and priority spending were adjusted in light of the revised 2011 budget law and updated information on the expected budget execution. Finally, the program now includes an adjuster for the reserve money AC to neutralize the impact of changes in reserve requirements, given that commercial banks' maintain very limited excess reserves.

\section{EXCHANGE RESTRICTIONS}

38. On May 20, 2011, the authorities accepted the obligations under Article VIII of the Fund's Articles of Agreement. They welcomed the cooperation with the Fund in identifying existing exchange restrictions and multiple currency practices and in proposing ways to eliminate them. New foreign exchange regulations have become effective, and the BM intends to adopt MEFP $¥ 16$ implementation norms shortly to eliminate all of the remaining exchange restrictions on the making of payments and transfers for current international transactions. The BM has also taken formal decisions to eliminate existing multiple currency practices (see Supplement 2).

\section{STAFf Appraisal}

39. Mozambique's economic performance remains strong. The country was less affected by the global crisis than many others on the continent. Helped by appropriate countercyclical policies, it managed to keep economic growth buoyant and benefitted from a quick recovery of its balance of payments.

\section{Although Mozambique is poised for sustained economic growth going forward,} significant challenges remain. In the short run, the authorities need to move decisively to prevent inflation expectations from becoming entrenched. Their resolve to further tighten fiscal and monetary policy in 2011 to bring about an early decline in core inflation is thus welcome. However, the authorities should stand ready to tighten policies further should inflation pressures persist. The recently signed information agreement between the government and the BM should be conducive to enhancing the effectiveness of the policy stance and strengthening the BM's ability to keep reserve money growth in line with the programmed path. 


\section{The authorities' new PARP contains the right ingredients to allow more} Mozambicans to benefit from economic growth. The stagnation in income poverty reduction and the September 2010 unrest are clear warning signals that Mozambique's traditional growth model needs to be complemented by well-targeted efforts to broaden the country's productive and export base and create employment opportunities. The challenge will be in the implementation of the PARP, as extensive inter-ministerial coordination is required and underlying sectoral strategies need to be aligned and, in some cases, reinvigorated.

42. In this context, the challenges on fiscal policy are expected to rise appreciably. While development partners have accompanied Mozambique through the years with generous financial support, such support is likely to have peaked. The authorities need to carefully assess their options to create new fiscal space for their ambitious development agenda. Their prudent first steps to borrow at nonconcessional terms bode well in this regard and generate confidence that Mozambique's strong track record of macroeconomic stability and debt sustainability will be preserved. But the true challenge will be to sustain the strong tax administration effort and tap new revenue sources, including in particular from the natural resource sector. The current public debate on this question should pave the way for a sensible solution that balances Mozambique as a destination for foreign investors and the need to have mega-projects provide a fair contribution to the country's development.

\section{The authorities need to ensure that social protection systems are expanded} while becoming well-targeted and affordable. Staff welcomes the announcement to continue with the gradual phase-out of the fuel subsidy which has been overly costly and negatively affected Mozambique's traditionally strong prioritization of government spending. However, care needs to be taken that those truly in need are supported by efficient safety nets - for which adequate fiscal space should become available over time. The authorities' plans to create food and transport vouchers carry some risks, especially with respect to coverage and administrative obstacles and costs. The authorities are advised to seek the expertise of development partners and other stakeholders to get any expansions to the social safety net right, so as to truly target the most vulnerable and contain risks to social peace.

\section{The banking system has shown resilience during the global crisis, but vigilance} is warranted in light of vulnerabilities. The authorities are urged to ensure adequate capitalization of all banks, tackle the persistent concentration risk in banks' balance sheets, and carefully monitor intra-group financial flows with parent banks abroad. While the progress in establishing a banking crisis resolution framework is commendable, more resolve needs to be shown to make the AML/CFT framework more effective, as a lack of action could entail serious reputational risks detrimental to investment and growth and endanger Mozambique's donor relations. 
45. The authorities' acceptance of Article VIII status is welcome. They have shown commendable diligence in removing existing exchange restrictions and potential multiple currency practices.

46. Based on the strong program performance, staff recommends completion of the second review under the PSI. It also recommends the modification of the end-June $2011 \mathrm{AC}$ and a waiver for the non-observance of the end-2010 AC on reserve money, since corrective action was taken through the information sharing agreement between the government and the BM.

47. It is proposed that the next Article IV consultation take place within 24 months, subject to the provisions of the decision on consultation cycles in program countries. 
Table 1. Mozambique: Selected Economic and Financial Indicators, 2009-16

\begin{tabular}{|c|c|c|c|c|c|c|c|c|c|c|}
\hline & \multirow{2}{*}{$\begin{array}{r}2009 \\
\text { Act. }\end{array}$} & \multicolumn{2}{|l|}{2010} & \multicolumn{2}{|l|}{2011} & 2012 & \multirow[t]{2}{*}{2013} & \multirow{2}{*}{$\begin{array}{c}2014 \\
\text { Proj. }\end{array}$} & \multirow[t]{2}{*}{2015} & \multirow[t]{2}{*}{2016} \\
\hline & & CR No. $10 / 375$ & Est. & CR No. $10 / 375$ & Proj. & & & & & \\
\hline & \multicolumn{9}{|c|}{ (Annual percentage change, unless otherwise indicated) } & \\
\hline \multicolumn{11}{|l|}{ National income and prices } \\
\hline Nominal GDP (MT billion) & 269 & 326 & 323 & 378 & 380 & 439 & 502 & 574 & 655 & 747 \\
\hline Nominal GDP growth & 12.1 & 20.9 & 20.1 & 16.1 & 17.4 & 15.7 & 14.3 & 14.2 & 14.2 & 14.1 \\
\hline Real GDP growth & 6.3 & 7.2 & 6.6 & 7.5 & 7.2 & 7.5 & 7.9 & 7.8 & 7.8 & 7.8 \\
\hline GDP per capita (US\$) & 477 & 453 & 454 & 477 & 550 & 603 & 657 & 708 & 755 & 812 \\
\hline Consumer price index (annual average) & 3.3 & 12.7 & 12.7 & 8.0 & 9.5 & 7.2 & 5.6 & 5.6 & 5.6 & 5.6 \\
\hline Consumer price index (end of period) & 4.2 & 15.5 & 16.6 & 6.9 & 8.4 & 5.6 & 5.6 & 5.6 & 5.6 & 5.6 \\
\hline Exchange rate, MT per US dollar, eop & 27.5 & 36.0 & 32.8 & $\ldots$ & $\ldots$ & $\ldots$ & $\ldots$ & $\ldots$ & $\ldots$ & $\ldots$ \\
\hline Exchange rate, MT per US dollar, per.avg. & 26.7 & 33.3 & 33.0 & $\ldots$ & $\ldots$ & $\ldots$ & $\ldots$ & $\ldots$ & $\ldots$ & $\ldots$ \\
\hline \multicolumn{11}{|l|}{ External sector } \\
\hline Merchandise exports & -19.1 & 8.4 & 4.5 & 20.5 & 14.8 & 17.3 & 10.9 & 10.5 & 10.5 & 10.0 \\
\hline Merchandise exports, excluding megaprojects & 4.3 & -23.0 & -31.3 & 2.7 & 6.9 & 3.4 & 4.9 & 5.4 & 5.3 & 5.6 \\
\hline Merchandise imports & -6.1 & 11.7 & -2.5 & 4.1 & 16.1 & 10.8 & 9.3 & 9.0 & 9.1 & 9.1 \\
\hline Merchandise imports, excluding megaprojects & -10.6 & 11.7 & -7.4 & 1.5 & 16.4 & 12.6 & 10.3 & 10.2 & 10.2 & 10.4 \\
\hline Terms of trade & -0.7 & 2.5 & 15.0 & 13.1 & 4.9 & 5.2 & -0.3 & -3.4 & -1.8 & 1.2 \\
\hline Nominal effective exchange rate (end of period) & -7.1 & $\ldots$ & -22.4 & $\ldots$ & $\ldots$ & $\ldots$ & $\ldots$ & $\ldots$ & $\ldots$ & $\ldots$ \\
\hline \multirow[t]{2}{*}{ Real effective exchange rate (end of period) } & -6.3 & $\ldots$ & -15.0 & $\ldots$ & $\ldots$ & $\ldots$ & $\ldots$ & $\ldots$ & $\ldots$ & $\ldots$ \\
\hline & \multicolumn{9}{|c|}{ (Annual percentage change, unless otherwise indicated) } & \\
\hline \multicolumn{11}{|l|}{ Money and credit } \\
\hline Reserve money & 27.3 & 25.0 & 29.2 & 13.0 & 14.3 & 16.9 & 18.7 & 22.0 & 22.2 & 22.8 \\
\hline M3 (Broad Money) & 32.6 & 27.0 & 22.8 & 18.3 & 20.0 & 23.1 & 23.3 & 25.2 & 25.5 & 26.7 \\
\hline Credit to the economy & 58.6 & 36.0 & 27.5 & 22.6 & 19.5 & 20.0 & 20.9 & 21.0 & 22.1 & 22.5 \\
\hline \multirow[t]{2}{*}{ (Percent of GDP) } & 26.5 & 29.8 & 28.2 & 31.5 & 28.6 & 29.7 & 31.4 & 33.3 & 35.6 & 38.3 \\
\hline & \multicolumn{9}{|c|}{ (Percent of GDP) } & \\
\hline \multicolumn{11}{|l|}{ Investment and saving } \\
\hline Gross domestic investment & 16.3 & 21.8 & 20.6 & 23.0 & 21.3 & 21.5 & 22.5 & 23.2 & 23.3 & 23.5 \\
\hline Government & 12.8 & 13.9 & 12.6 & 13.1 & 11.7 & 11.4 & 11.8 & 11.9 & 11.7 & 12.0 \\
\hline Other sectors & 3.5 & 8.0 & 8.0 & 9.9 & 9.6 & 10.1 & 10.7 & 11.2 & 11.5 & 11.5 \\
\hline Gross domestic savings (excluding grants) & -2.6 & 1.2 & 4.3 & 3.2 & 4.3 & 5.3 & 6.5 & 7.4 & 7.7 & 8.3 \\
\hline Government & -0.4 & -0.2 & 1.2 & 0.7 & 1.0 & 0.8 & 1.3 & 1.5 & 1.5 & 1.7 \\
\hline Other sectors & -2.2 & 1.4 & 3.1 & 2.5 & 3.3 & 4.5 & 5.2 & 5.9 & 6.1 & 6.6 \\
\hline External current account, before grants & -18.9 & -20.7 & -16.4 & -19.8 & -17.0 & -16.2 & -16.1 & -15.8 & -15.6 & -15.2 \\
\hline External current account, after grants & -12.1 & -13.4 & -10.2 & -12.0 & -11.4 & -10.9 & -10.9 & -10.7 & -10.6 & -10.5 \\
\hline Government budget & & & & & & & & & & \\
\hline Total revenue & 17.4 & 18.4 & 19.6 & 18.9 & 20.2 & 20.6 & 21.1 & 21.4 & 21.5 & 21.8 \\
\hline Total expenditure and net lending & 32.2 & 32.5 & 31.4 & 35.3 & 33.9 & 34.4 & 34.5 & 34.1 & 33.7 & 33.8 \\
\hline Overall balance, before grants & -14.8 & -14.1 & -11.8 & -16.4 & -13.7 & -13.8 & -13.4 & -12.7 & -12.2 & -12.0 \\
\hline Total grants & 9.4 & 9.2 & 8.1 & 9.4 & 7.3 & 7.1 & 7.0 & 6.9 & 6.8 & 6.7 \\
\hline Overall balance, after grants & -5.4 & -4.9 & -3.7 & -7.0 & -6.4 & -6.8 & -6.4 & -5.9 & -5.4 & -5.3 \\
\hline Domestic primary balance, before grants & -4.3 & -4.1 & -3.2 & -3.3 & -3.1 & -3.4 & -3.2 & -2.8 & -2.6 & -2.5 \\
\hline External financing (incl. debt relief) & 5.0 & 4.5 & 4.3 & 6.8 & 5.8 & 5.8 & 5.5 & 4.9 & 4.6 & 4.5 \\
\hline Net domestic financing & 0.2 & 0.3 & -0.5 & 0.2 & 0.5 & 1.0 & 0.9 & 0.9 & 0.8 & 0.8 \\
\hline Privatization & 0.2 & 0.1 & 0.0 & 0.0 & 0.0 & 0.0 & 0.0 & 0.0 & 0.0 & 0.0 \\
\hline Total public debt & 38.2 & $\ldots$ & 39.3 & $\ldots$ & $\ldots$ & $\ldots$ & $\ldots$ & $\ldots$ & $\ldots$ & $\ldots$ \\
\hline Of which : external & 35.4 & $\ldots$ & 35.2 & $\ldots$ & $\ldots$ & $\ldots$ & $\ldots$ & $\ldots$ & $\ldots$ & $\ldots$ \\
\hline Of which : domestic & 2.8 & $\ldots$ & 4.1 & $\ldots$ & $\ldots$ & $\ldots$ & $\ldots$ & $\ldots$ & $\ldots$ & $\ldots$ \\
\hline & & & $(U S \$ n$ & llions, unless oth & erwise in & cated) & & & & \\
\hline External current account, before grants & $-1,908$ & $-2,024$ & $-1,603$ & $-2,082$ & $-2,055$ & $-2,195$ & $-2,417$ & $-2,609$ & $-2,808$ & $-3,004$ \\
\hline External current account, after grants & $-1,220$ & $-1,308$ & -999 & $-1,258$ & $-1,380$ & $-1,478$ & $-1,637$ & $-1,770$ & $-1,908$ & $-2,077$ \\
\hline Overall balance of payments & 194 & -157 & 58 & 220 & 91 & 302 & 259 & 368 & 416 & 458 \\
\hline Net international reserves (end of period) 1 & 1,840 & 1,675 & 1,908 & 1,895 & 2,000 & 2,302 & 2,560 & 2,928 & 3,345 & 3,802 \\
\hline Gross international reserves (end of period) 1 & 2,012 & 1,868 & 2,099 & 2,086 & 2,189 & 2,487 & 2,743 & 3,077 & 3,432 & 3,823 \\
\hline Months of projected imports of goods and nonfactor services & 5.4 & 4.4 & 4.8 & 4.5 & 4.5 & 4.7 & 4.7 & 4.8 & 4.9 & 5.0 \\
\hline
\end{tabular}

Sources: Mozambican authorities; and IMF staff estimates and projections.

${ }^{1}$ Includes disbursements of IMF resources under the ESF and August 2009 SDR allocation. 
Table 2. Mozambique: Government Finances, 2009-11

(MT Billions)

\begin{tabular}{|c|c|c|c|c|c|}
\hline & 2009 & \multicolumn{2}{|l|}{2010} & \multicolumn{2}{|l|}{2011} \\
\hline & Act. & CR No. $10 / 375$ & Est. & CR No. $10 / 375$ & Proj. $/ 1$ \\
\hline Total revenue & 46.73 & 59.94 & 63.49 & 71.45 & 76.79 \\
\hline Tax revenue & 41.47 & 53.31 & 56.47 & 63.22 & 67.56 \\
\hline Income and profits & 13.72 & 18.40 & 18.50 & 23.58 & 22.01 \\
\hline Goods and services & 22.89 & 29.06 & 31.63 & 32.44 & 37.69 \\
\hline International trade & 4.08 & 4.59 & 5.24 & 5.85 & 6.53 \\
\hline Other & 0.78 & 1.26 & 1.10 & 1.35 & 1.33 \\
\hline Nontax revenue & 5.26 & 6.63 & 7.02 & 8.23 & 9.23 \\
\hline Total expenditure and net lending & 86.70 & 105.87 & 103.91 & 133.39 & 128.93 \\
\hline Current expenditure & 47.87 & 60.56 & 59.64 & 68.78 & 72.99 \\
\hline Compensation to employees & 23.62 & 29.56 & 29.11 & 36.25 & 36.25 \\
\hline Goods and services & 11.72 & 12.40 & 13.17 & 14.47 & 16.85 \\
\hline Interest on public debt & 1.36 & 2.76 & 2.65 & 3.59 & 3.31 \\
\hline Transfer payments 2/ & 11.17 & 15.83 & 14.71 & 14.47 & 16.58 \\
\hline Of which : fuel subsidy (old stock) $3 /$ & 2.80 & 4.13 & 4.69 & $\ldots$ & 3.62 \\
\hline new emergency spending (food voucher) 4/ & $\ldots$ & $\ldots$ & $\ldots$ & $\cdots$ & 0.34 \\
\hline new emergency spending (transport voucher) 4/ & $\ldots$ & $\ldots$ & $\ldots$ & $\ldots$ & 0.20 \\
\hline Domestic current primary balance & 0.22 & 2.15 & 6.51 & 6.26 & 7.11 \\
\hline Capital expenditure & 34.41 & 45.14 & 40.86 & 49.59 & 44.27 \\
\hline Domestically financed & 11.72 & 16.62 & 19.11 & 18.42 & 18.77 \\
\hline Externally financed & 22.69 & 28.52 & 21.75 & 31.17 & 25.50 \\
\hline Net lending & 4.42 & 0.17 & 3.42 & 15.01 & 11.68 \\
\hline Domestically financed & -0.02 & -1.01 & -0.13 & 0.19 & 0.19 \\
\hline Externally financed loans to public enterprises & 4.44 & 1.18 & 3.54 & 14.82 & 11.48 \\
\hline of which : nonconcessional net lending & 0.00 & 0.00 & 0.00 & 10.79 & 7.84 \\
\hline Unallocated revenue (+)/expenditure (-) 5/ & 0.00 & 0.00 & 2.21 & 0.00 & 0.00 \\
\hline Domestic primary balance, before grants, above the line 6/ & -11.47 & -13.46 & -10.27 & -12.35 & -11.86 \\
\hline Overall balance, before grants & -39.97 & -45.93 & -38.22 & -61.94 & -52.14 \\
\hline Grants received & 25.30 & 29.94 & 26.16 & 35.53 & 27.91 \\
\hline Project support & 16.18 & 17.22 & 14.16 & 23.40 & 17.39 \\
\hline Investment projects & 7.17 & 7.03 & 6.54 & 7.66 & 7.29 \\
\hline Special programs & 8.64 & 10.19 & 7.62 & 15.74 & 10.11 \\
\hline Direct financing & 0.37 & 0.00 & 0.00 & 0.00 & 0.00 \\
\hline Budget support & 9.12 & 12.72 & 12.00 & 12.13 & 10.52 \\
\hline Overall balance, after grants & -14.67 & -15.99 & -12.06 & -26.40 & -24.24 \\
\hline Net external financing & 13.50 & 14.62 & 13.81 & 25.59 & 22.15 \\
\hline Disbursements & 14.20 & 16.21 & 14.85 & 26.77 & 23.26 \\
\hline Project & 6.51 & 11.31 & 7.59 & 7.77 & 8.11 \\
\hline Nonproject support & 7.69 & 4.91 & 7.26 & 19.00 & 15.15 \\
\hline Loans to public enterprises & 3.89 & 1.18 & 3.54 & 14.82 & 11.48 \\
\hline Budget support & 3.80 & 3.73 & 3.71 & 4.18 & 3.67 \\
\hline Cash amortization & -0.70 & -1.60 & -1.03 & -1.18 & -1.11 \\
\hline Net domestic financing & 0.52 & 1.04 & -1.74 & 0.81 & 2.09 \\
\hline Net privatization & 0.41 & 0.33 & -0.01 & 0.00 & 0.00 \\
\hline \multicolumn{6}{|l|}{ Memorandum items: } \\
\hline Net aid flows & 38.26 & 43.72 & 39.16 & 49.40 & 41.21 \\
\hline Gross aid flows & 39.50 & 46.15 & 41.00 & 51.51 & 43.33 \\
\hline Budget support & 12.92 & 16.45 & 15.71 & 16.31 & 14.19 \\
\hline Nonbudget support & 26.58 & 29.70 & 25.30 & 35.20 & 29.15 \\
\hline Project support & 22.69 & 28.52 & 21.75 & 31.17 & 25.50 \\
\hline Loans to public enterprises & 3.89 & 1.18 & 3.54 & 4.03 & 3.65 \\
\hline External debt service & 1.24 & 2.44 & 1.85 & 2.11 & 2.12 \\
\hline
\end{tabular}

${ }^{1}$ In line with the organic budget law (SISTAFE 2002) budget execution will be limited to cautionary ceilings untill evidence of stronger revenue performance materializes.

${ }^{2}$ Includes the cost in of reverting the planned elimination of selected food and urban tranport subsidy (MT $0.5 \mathrm{bn}$ in 2010 and MT $1.2 \mathrm{bn}$ in 2011).

${ }^{3}$ In 2011, covers the full repayment of the end-2010 stock of accumulated losses of fuel importers (USD $127 \mathrm{mln}$ ), reflecting an agreement between the government, and the fuel importers.

${ }^{4}$ In 2011, covers the following emergency measures for 2011H2: (1) a food voucher program ( cesta basica - MT $334 \mathrm{mIn}$ ) and (2) a tranport voucher program (MT $200 \mathrm{mIn}$ ),

both to be included in the supplementary budget.

${ }^{5}$ Residual discrepancy between identified sources and uses of funds.

${ }^{6}$ Revenue minus noninterest current expenditure minus locally financed capital expenditure and locally financed net lending. 
Table 3. Mozambique: Government Finances, 2009-16 (Percent of GDP)

\begin{tabular}{|c|c|c|c|c|c|c|c|c|c|c|}
\hline & \multirow{2}{*}{$\begin{array}{l}2009 \\
\text { Act. }\end{array}$} & \multicolumn{2}{|l|}{2010} & \multicolumn{2}{|l|}{2011} & \multirow[t]{2}{*}{2012} & \multirow[t]{2}{*}{2013} & \multirow{2}{*}{$\begin{array}{l}2014 \\
\text { Proj. }\end{array}$} & \multirow[t]{2}{*}{2015} & \multirow[t]{2}{*}{2016} \\
\hline & & CR No. $10 / 375$ & Est. & CR No. $10 / 375$ & Proj. 1/ & & & & & \\
\hline Total revenue & 17.4 & 18.4 & 19.6 & 18.9 & 20.2 & 20.6 & 21.1 & 21.4 & 21.5 & 21.8 \\
\hline Tax revenue & 15.4 & 16.4 & 17.5 & 16.7 & 17.8 & 18.2 & 18.7 & 19.0 & 19.1 & 19.4 \\
\hline Nontax revenue & 2.0 & 2.0 & 2.2 & 2.2 & 2.4 & 2.4 & 2.4 & 2.4 & 2.4 & 2.4 \\
\hline Total expenditure and net lending & 32.2 & 32.5 & 31.4 & 35.3 & 33.9 & 34.4 & 34.5 & 34.1 & 33.7 & 33.8 \\
\hline Current expenditure & 17.8 & 18.6 & 18.4 & 18.2 & 19.2 & 19.8 & 19.8 & 19.9 & 20.0 & 20.1 \\
\hline Compensation to employees & 8.8 & 9.1 & 9.0 & 9.6 & 9.5 & 9.4 & 9.2 & 9.1 & 8.8 & 8.8 \\
\hline Goods and services & 4.3 & 3.8 & 4.1 & 3.8 & 4.4 & 4.9 & 4.9 & 4.9 & 5.0 & 5.1 \\
\hline Interest on public debt & 0.5 & 0.8 & 0.8 & 1.0 & 0.9 & 0.8 & 0.8 & 1.0 & 1.1 & 1.2 \\
\hline Domestic & 0.3 & 0.6 & 0.6 & 0.7 & 0.6 & 0.4 & 0.3 & 0.4 & 0.4 & 0.5 \\
\hline External & 0.2 & 0.3 & 0.3 & 0.2 & 0.3 & 0.4 & 0.5 & 0.6 & 0.7 & 0.7 \\
\hline Transfer payments 2/ & 4.1 & 4.9 & 4.5 & 3.8 & 4.4 & 4.7 & 4.9 & 4.9 & 5.1 & 5.1 \\
\hline Of which: fuel subsidy (old stock) 3/ & 1.0 & 1.3 & 1.5 & $\ldots$ & 1.0 & $\ldots$ & $\ldots$ & $\ldots$ & $\ldots$ & $\ldots$ \\
\hline new emergency spending (food voucher) 4/ & $\ldots$ & $\ldots$ & $\ldots$ & $\ldots$ & 0.1 & $\ldots$ & $\ldots$ & $\ldots$ & $\ldots$ & $\ldots$ \\
\hline new emergency spending (transport voucher) 4/ & $\ldots$ & $\ldots$ & $\ldots$ & $\ldots$ & 0.1 & $\cdots$ & $\cdots$ & $\cdots$ & $\cdots$ & $\cdots$ \\
\hline Capital expenditure & 12.8 & 13.9 & 12.6 & 13.1 & 11.7 & 11.4 & 11.8 & 11.9 & 11.7 & 12.0 \\
\hline Domestically financed & 4.4 & 5.1 & 5.9 & 4.9 & 4.9 & 5.0 & 5.2 & 5.3 & 5.2 & 5.4 \\
\hline Externally financed & 8.4 & 8.8 & 6.7 & 8.2 & 6.7 & 6.4 & 6.6 & 6.6 & 6.6 & 6.6 \\
\hline Net lending & 1.6 & 0.1 & 1.1 & 4.0 & 3.1 & 3.2 & 2.8 & 2.3 & 2.0 & 1.7 \\
\hline Domestically financed & 0.0 & -0.3 & 0.0 & 0.1 & 0.1 & 0.1 & 0.0 & 0.0 & 0.1 & 0.1 \\
\hline Externally financed loans to public enterprises & 1.7 & 0.4 & 1.1 & 3.9 & 3.0 & 3.2 & 2.7 & 2.3 & 1.9 & 1.6 \\
\hline of which: concessional & 1.7 & 0.4 & 1.1 & 1.1 & 1.0 & 1.0 & 0.9 & 0.9 & 0.9 & 0.9 \\
\hline of which: nonconcessional net lending & 0.0 & 0.0 & 0.0 & 2.9 & 2.1 & 2.2 & 1.8 & 1.3 & 1.0 & 0.7 \\
\hline Unallocated revenue (+)/expenditure (-) 5/ & 0.0 & 0.0 & 0.7 & 0.0 & 0.0 & 0.0 & 0.0 & 0.0 & 0.0 & 0.0 \\
\hline Domestic primary balance, before grants 6 / & -4.3 & -4.1 & -3.2 & -3.3 & -3.1 & -3.4 & -3.2 & -2.8 & -2.6 & -2.5 \\
\hline Overall balance, before grants & -14.8 & -14.1 & -11.8 & -16.4 & -13.7 & -13.8 & -13.4 & -12.7 & -12.2 & -12.0 \\
\hline Grants received & 9.4 & 9.2 & 8.1 & 9.4 & 7.3 & 7.1 & 7.0 & 6.9 & 6.8 & 6.7 \\
\hline Project & 6.0 & 5.3 & 4.4 & 6.2 & 4.6 & 4.4 & 4.4 & 4.3 & 4.2 & 4.1 \\
\hline Investment projects & 2.7 & 2.2 & 2.0 & 2.0 & 1.9 & 1.9 & 1.9 & 1.9 & 1.9 & 2.1 \\
\hline Special programs & 3.2 & 3.1 & 2.4 & 4.2 & 2.7 & 2.5 & 2.5 & 2.4 & 2.3 & 2.0 \\
\hline Direct financing & 0.1 & 0.0 & 0.0 & 0.0 & 0.0 & 0.0 & 0.0 & 0.0 & 0.0 & 0.0 \\
\hline Nonproject & 3.4 & 3.9 & 3.7 & 3.2 & 2.8 & 2.7 & 2.6 & 2.6 & 2.6 & 2.6 \\
\hline Overall balance, after grants & -5.4 & -4.9 & -3.7 & -7.0 & -6.4 & -6.8 & -6.4 & -5.9 & -5.4 & -5.3 \\
\hline Net external financing & 5.0 & 4.5 & 4.3 & 6.8 & 5.8 & 5.8 & 5.5 & 4.9 & 4.6 & 4.5 \\
\hline Disbursements & 5.3 & 5.0 & 4.6 & 7.1 & 6.1 & 6.3 & 6.3 & 6.0 & 5.8 & 5.9 \\
\hline Project & 2.4 & 3.5 & 2.3 & 2.1 & 2.1 & 2.0 & 2.2 & 2.3 & 2.3 & 2.5 \\
\hline Nonproject support & 2.9 & 1.5 & 2.2 & 5.0 & 4.0 & 4.3 & 4.0 & 3.7 & 3.5 & 3.3 \\
\hline Loans to public enterprises & 1.4 & 0.4 & 1.1 & 3.9 & 3.0 & 3.4 & 3.1 & 2.7 & 2.5 & 2.4 \\
\hline of which: nonconcessional & 0.0 & 0.0 & 0.0 & 2.9 & 2.1 & 2.4 & 2.2 & 1.8 & 1.6 & 1.5 \\
\hline Budget support & 1.4 & 1.1 & 1.1 & 1.1 & 1.0 & 1.0 & 1.0 & 1.0 & 1.0 & 1.0 \\
\hline Cash amortization & -0.3 & -0.5 & -0.3 & -0.3 & -0.3 & -0.5 & -0.7 & -1.1 & -1.2 & -1.3 \\
\hline Net domestic financing & 0.2 & 0.3 & -0.5 & 0.2 & 0.5 & 1.0 & 0.9 & 0.9 & 0.8 & 0.8 \\
\hline Net privatization & 0.2 & 0.1 & 0.0 & 0.0 & 0.0 & 0.0 & 0.0 & 0.0 & 0.0 & 0.0 \\
\hline \multicolumn{11}{|l|}{ Memorandum items: } \\
\hline Net aid flows & 14.5 & 13.4 & 12.1 & 13.1 & 10.8 & 10.1 & 9.8 & 9.3 & 9.1 & 9.0 \\
\hline Gross aid flows & 15.0 & 14.2 & 12.7 & 13.6 & 11.4 & 11.0 & 11.1 & 11.0 & 11.0 & 11.1 \\
\hline Budget support & 4.9 & 5.1 & 4.9 & 4.3 & 3.7 & 3.6 & 3.6 & 3.5 & 3.5 & 3.5 \\
\hline Nonbudget support & 10.1 & 9.1 & 7.8 & 9.3 & 7.7 & 7.4 & 7.5 & 7.5 & 7.5 & 7.6 \\
\hline Project support & 8.6 & 8.8 & 6.7 & 8.2 & 6.7 & 6.4 & 6.6 & 6.6 & 6.6 & 6.6 \\
\hline Loans to public enterprises & 1.5 & 0.4 & 1.1 & 1.1 & 1.0 & 1.0 & 0.9 & 0.9 & 0.9 & 0.9 \\
\hline External debt service & 0.5 & 0.7 & 0.6 & 0.6 & 0.6 & 0.9 & 1.3 & 1.7 & 1.9 & 2.1 \\
\hline
\end{tabular}

Sources: Mozambican authorities; and IMF staff estimates and projections.

${ }^{1}$ In line with the organic budget law (SISTAFE 2002) budget execution will be limited to cautionary ceilings untill evidence of stronger revenue performance materializes. ${ }^{2}$ Includes the cost of reverting the planned elimination of selected food and urban tranport subsidy (MT $0.5 \mathrm{bn}$ in 2010 and MT $1.2 \mathrm{bn}$ in 2011).

${ }^{3}$ In 2011, covers the full repayment of the end-2010 stock of accumulated losses of fuel importers (USD $127 \mathrm{~m} / \mathrm{n}$ ), reflecting an agreement between the government, and the fuel importers. ${ }^{4}$ In 2011, covers the following emergency measures for 2011H2: (1) a food voucher program (cesta basica - MT $334 \mathrm{mIn}$ ) and (2) a tranport voucher program (MT $200 \mathrm{mln}$ ), both to be included in the supplementary budget.

${ }^{5}$ Residual discrepancy between identified sources and uses of funds.

${ }^{6}$ Revenue minus noninterest current expenditure minus locally financed capital expenditure and locally financed net lending. 
Table 4. Mozambique: Monetary Survey, Quarterly, 2009-11

\begin{tabular}{|c|c|c|c|c|c|c|c|c|c|c|c|c|c|}
\hline & \multirow{3}{*}{\begin{tabular}{|l|}
2009 \\
Q4 \\
Act. \\
\end{tabular}} & \multicolumn{8}{|c|}{2010} & \multicolumn{4}{|c|}{2011} \\
\hline & & \multicolumn{2}{|c|}{ Q1 } & \multicolumn{2}{|c|}{ Q2 } & \multicolumn{2}{|l|}{ Q3 } & \multicolumn{2}{|l|}{ Q4 } & Q1 & \multirow{2}{*}{\multicolumn{2}{|c|}{$\begin{array}{lc}\text { Q2 } & \text { Q3 } \\
& \text { Projections } \\
\end{array}$}} & \multirow[t]{2}{*}{ Q4 } \\
\hline & & Prog. & Act. & Prog. & Act. & $\begin{array}{l}\text { Prog. } \\
\end{array}$ & Act. C & CR No. 10/375 & Act. & CR No. 10/375 & & & \\
\hline & \multicolumn{13}{|c|}{ (MT Billions, unless otherwise stated) } \\
\hline \multicolumn{14}{|l|}{ Bank of Mozambique } \\
\hline $\begin{array}{l}\text { Net foreign assets } \\
\text { (US\$ billions) } \\
\text { Net international reserves } \\
\text { (US\$ billions) }\end{array}$ & $\begin{array}{r}49.6 \\
1.7 \\
53.7 \\
1.8\end{array}$ & $\begin{array}{r}45.2 \\
1.6 \\
48.8 \\
1.7\end{array}$ & $\begin{array}{r}47.6 \\
1.5 \\
52.4 \\
1.7\end{array}$ & $\begin{array}{r}49.8 \\
1.6 \\
53.5 \\
1.8\end{array}$ & $\begin{array}{r}56.3 \\
1.6 \\
61.0 \\
1.7\end{array}$ & $\begin{array}{r}54.5 \\
1.8 \\
58.3 \\
1.9\end{array}$ & $\begin{array}{r}58.9 \\
1.6 \\
64.4 \\
1.8\end{array}$ & $\begin{array}{r}55.5 \\
1.5 \\
60.4 \\
1.7\end{array}$ & $\begin{array}{r}56.9 \\
1.7 \\
62.2 \\
1.9\end{array}$ & $\begin{array}{r}57.7 \\
1.6 \\
62.6 \\
1.7\end{array}$ & $\begin{array}{r}51.7 \\
1.7 \\
56.8 \\
1.8\end{array}$ & $\begin{array}{r}54.4 \\
1.7 \\
59.5 \\
1.9\end{array}$ & $\begin{array}{r}59.5 \\
1.8 \\
64.8 \\
2.0\end{array}$ \\
\hline $\begin{array}{l}\text { Net domestic assets } \\
\text { Credit to government (net) } \\
\text { Credit to banks (net) } \\
\text { Credit to the economy } \\
\text { Other items (net; assets +) }\end{array}$ & $\begin{array}{r}-25.2 \\
-25.3 \\
2.3 \\
0.4 \\
-2.6\end{array}$ & $\begin{array}{r}-22.7 \\
-18.7 \\
-0.5 \\
0.4 \\
-3.9\end{array}$ & $\begin{array}{r}-24.7 \\
-23.3 \\
1.7 \\
0.4 \\
-3.5\end{array}$ & $\begin{array}{r}-24.0 \\
-18.1 \\
-0.5 \\
0.4 \\
-5.9\end{array}$ & $\begin{array}{r}-30.2 \\
-24.9 \\
1.8 \\
0.5 \\
-7.6\end{array}$ & $\begin{array}{r}-28.7 \\
-22.4 \\
-0.5 \\
0.4 \\
-6.2\end{array}$ & $\begin{array}{r}-29.5 \\
-21.1 \\
1.9 \\
0.5 \\
-10.8\end{array}$ & $\begin{array}{r}-25.0 \\
-14.8 \\
-0.5 \\
0.5 \\
-10.1\end{array}$ & $\begin{array}{r}-25.2 \\
-20.6 \\
0.4 \\
0.5 \\
-5.6\end{array}$ & $\begin{array}{r}-30.2 \\
-19.3 \\
-0.5 \\
0.5 \\
-10.8\end{array}$ & $\begin{array}{r}-21.1 \\
-21.8 \\
2.5 \\
0.5 \\
-2.3\end{array}$ & $\begin{array}{r}-20.2 \\
-20.2 \\
2.4 \\
0.5 \\
-2.8\end{array}$ & $\begin{array}{r}-23.4 \\
-18.5 \\
1.6 \\
0.5 \\
-6.9\end{array}$ \\
\hline $\begin{array}{l}\text { Reserve money } \\
\text { Currency in circulation } \\
\text { Bank deposits in BM }\end{array}$ & $\begin{array}{r}24.5 \\
16.1 \\
8.3\end{array}$ & $\begin{array}{r}22.5 \\
14.1 \\
8.5\end{array}$ & $\begin{array}{r}23.0 \\
14.5 \\
8.5\end{array}$ & $\begin{array}{r}25.7 \\
16.4 \\
9.3\end{array}$ & $\begin{array}{r}26.1 \\
16.7 \\
9.3\end{array}$ & $\begin{array}{r}25.8 \\
16.4 \\
9.3\end{array}$ & $\begin{array}{l}29.4 \\
18.4 \\
11.0\end{array}$ & $\begin{array}{l}30.6 \\
19.1 \\
11.5\end{array}$ & $\begin{array}{l}31.6 \\
20.4 \\
11.2\end{array}$ & $\begin{array}{l}27.5 \\
16.1 \\
11.4\end{array}$ & $\begin{array}{l}30.6 \\
19.2 \\
11.4\end{array}$ & $\begin{array}{l}34.2 \\
22.5 \\
11.7\end{array}$ & $\begin{array}{l}36.2 \\
24.1 \\
12.0\end{array}$ \\
\hline \multicolumn{14}{|l|}{ Commercial Banks } \\
\hline $\begin{array}{l}\text { Net foreign assets } \\
\quad \text { (in millions of U.S. dollars) }\end{array}$ & $\begin{array}{r}14.1 \\
0.5\end{array}$ & $\begin{array}{r}14.0 \\
0.5\end{array}$ & $\begin{array}{r}13.4 \\
0.4\end{array}$ & $\begin{array}{r}17.5 \\
0.6\end{array}$ & $\begin{array}{r}17.2 \\
0.5\end{array}$ & $\begin{array}{r}17.4 \\
0.6\end{array}$ & $\begin{array}{r}21.9 \\
0.6\end{array}$ & $\begin{array}{r}18.6 \\
0.5\end{array}$ & $\begin{array}{r}22.8 \\
0.7\end{array}$ & $\begin{array}{c}9.0 \\
0.2\end{array}$ & $\begin{array}{r}16.1 \\
0.5\end{array}$ & $\begin{array}{r}11.7 \\
0.4\end{array}$ & $\begin{array}{r}15.6 \\
0.5\end{array}$ \\
\hline $\begin{array}{l}\text { Net domestic assets } \\
\text { Banks' reserves } \\
\text { Credit to BM (net) } \\
\text { Credit to government (net) } \\
\text { Credit to the economy } \\
\text { Other items (net; assets +) }\end{array}$ & $\begin{array}{r}79.3 \\
11.7 \\
-2.5 \\
13.8 \\
71.0 \\
-14.8\end{array}$ & $\begin{array}{r}80.4 \\
10.1 \\
0.5 \\
12.6 \\
72.8 \\
-15.6\end{array}$ & $\begin{array}{r}82.4 \\
11.0 \\
-1.6 \\
14.9 \\
74.1 \\
-15.9\end{array}$ & $\begin{array}{r}87.4 \\
11.5 \\
0.5 \\
7.3 \\
84.3 \\
-16.1\end{array}$ & $\begin{array}{r}86.5 \\
11.8 \\
-1.8 \\
12.4 \\
82.4 \\
-18.3\end{array}$ & $\begin{array}{r}87.8 \\
11.3 \\
0.5 \\
14.2 \\
77.8 \\
-16.1\end{array}$ & $\begin{array}{r}89.6 \\
13.3 \\
-0.8 \\
7.0 \\
90.7 \\
-20.5\end{array}$ & $\begin{array}{r}101.0 \\
14.2 \\
0.5 \\
4.3 \\
96.7 \\
-14.6\end{array}$ & $\begin{array}{r}90.6 \\
14.3 \\
-0.3 \\
7.9 \\
90.6 \\
-21.9\end{array}$ & $\begin{array}{r}110.4 \\
13.1 \\
0.5 \\
10.2 \\
99.2 \\
-12.6\end{array}$ & $\begin{array}{r}110.9 \\
13.6 \\
-2.5 \\
8.3 \\
104.1 \\
-12.6\end{array}$ & $\begin{array}{r}118.4 \\
13.6 \\
-2.4 \\
7.3 \\
108.6 \\
-8.6\end{array}$ & $\begin{array}{r}117.6 \\
11.6 \\
-1.6 \\
7.9 \\
108.3 \\
-8.6\end{array}$ \\
\hline $\begin{array}{l}\text { Deposits } \\
\text { Demand and savings deposits } \\
\text { Time deposits }\end{array}$ & $\begin{array}{l}93.4 \\
60.2 \\
33.2\end{array}$ & $\begin{array}{l}94.4 \\
59.2 \\
35.2\end{array}$ & $\begin{array}{l}95.8 \\
60.3 \\
35.4\end{array}$ & $\begin{array}{r}104.9 \\
65.6 \\
39.4\end{array}$ & $\begin{array}{r}103.7 \\
63.9 \\
39.8\end{array}$ & $\begin{array}{r}105.3 \\
66.6 \\
38.6\end{array}$ & $\begin{array}{r}111.6 \\
71.5 \\
40.1\end{array}$ & $\begin{array}{r}119.6 \\
73.4 \\
46.2\end{array}$ & $\begin{array}{r}113.3 \\
71.1 \\
42.3\end{array}$ & $\begin{array}{r}119.4 \\
72.2 \\
47.2\end{array}$ & $\begin{array}{r}127.0 \\
79.9 \\
47.0\end{array}$ & $\begin{array}{r}130.1 \\
81.9 \\
48.2\end{array}$ & $\begin{array}{r}133.2 \\
82.1 \\
51.1\end{array}$ \\
\hline \multicolumn{14}{|l|}{ Monetary Survey } \\
\hline $\begin{array}{l}\text { Net foreign assets } \\
\text { (US } \$ \text { billions) }\end{array}$ & $\begin{array}{r}63.7 \\
2.2\end{array}$ & $\begin{array}{r}59.2 \\
2.0\end{array}$ & $\begin{array}{r}61.0 \\
2.0\end{array}$ & $\begin{array}{r}67.3 \\
2.2\end{array}$ & $\begin{array}{r}73.5 \\
2.1\end{array}$ & $\begin{array}{r}71.9 \\
2.3\end{array}$ & $\begin{array}{r}80.8 \\
2.2\end{array}$ & $\begin{array}{r}74.1 \\
2.1\end{array}$ & $\begin{array}{r}79.6 \\
2.4\end{array}$ & $\begin{array}{r}66.7 \\
1.8\end{array}$ & $\begin{array}{r}67.8 \\
2.2\end{array}$ & $\begin{array}{r}66.1 \\
2.1\end{array}$ & $\begin{array}{r}75.2 \\
2.3\end{array}$ \\
\hline $\begin{array}{l}\text { Net domestic assets } \\
\text { Domestic credit } \\
\text { Credit to government (net) } \\
\text { Credit to the economy } \\
\text { Cred. economy in foreign currency } \\
\text { Other items (net; assets +) }\end{array}$ & $\begin{array}{r}43.3 \\
60.0 \\
-11.5 \\
71.4 \\
22.8 \\
-16.6\end{array}$ & $\begin{array}{r}47.6 \\
67.2 \\
-6.1 \\
73.2 \\
25.5 \\
-19.6\end{array}$ & $\begin{array}{r}47.3 \\
66.1 \\
-8.5 \\
74.5 \\
25.3 \\
-18.8\end{array}$ & $\begin{array}{r}51.9 \\
73.9 \\
-10.8 \\
84.7 \\
28.6 \\
-22.0\end{array}$ & $\begin{array}{r}45.0 \\
70.4 \\
-12.5 \\
82.8 \\
29.5 \\
-25.3\end{array}$ & $\begin{array}{r}47.7 \\
70.0 \\
-8.2 \\
78.2 \\
25.8 \\
-22.3\end{array}$ & $\begin{array}{r}47.2 \\
77.0 \\
-14.1 \\
91.2 \\
29.8 \\
-29.8\end{array}$ & $\begin{array}{r}61.9 \\
86.7 \\
-10.5 \\
97.1 \\
34.0 \\
-24.8\end{array}$ & $\begin{array}{r}51.8 \\
78.3 \\
-12.7 \\
91.1 \\
21.2 \\
-26.5\end{array}$ & $\begin{array}{r}67.2 \\
90.6 \\
-9.1 \\
99.7 \\
35.9 \\
-23.4\end{array}$ & $\begin{array}{r}76.1 \\
91.1 \\
-13.5 \\
104.6 \\
32.0 \\
-14.9\end{array}$ & $\begin{array}{r}84.6 \\
96.1 \\
-13.0 \\
109.1 \\
33.6 \\
-11.5\end{array}$ & $\begin{array}{r}82.6 \\
98.1 \\
-10.7 \\
108.8 \\
31.9 \\
-15.5\end{array}$ \\
\hline $\begin{array}{l}\text { Money and quasi money (M3) } \\
\text { Foreign currency deposits } \\
\text { (US } \$ \text { billions) } \\
\text { M2 }\end{array}$ & $\begin{array}{r}107.1 \\
34.5 \\
1.2 \\
72.5\end{array}$ & $\begin{array}{r}106.8 \\
35.5 \\
1.2 \\
71.3\end{array}$ & $\begin{array}{r}108.3 \\
37.2 \\
1.2 \\
71.1\end{array}$ & $\begin{array}{r}119.2 \\
39.6 \\
1.3 \\
79.6\end{array}$ & $\begin{array}{r}118.5 \\
42.2 \\
1.2 \\
76.3\end{array}$ & $\begin{array}{r}119.7 \\
38.4 \\
1.2 \\
81.3\end{array}$ & $\begin{array}{r}128.0 \\
48.5 \\
1.3 \\
79.5\end{array}$ & $\begin{array}{r}136.0 \\
45.0 \\
1.2 \\
91.0\end{array}$ & $\begin{array}{r}131.5 \\
46.2 \\
1.4 \\
85.3\end{array}$ & $\begin{array}{r}133.9 \\
43.1 \\
1.2 \\
90.8\end{array}$ & $\begin{array}{r}143.9 \\
53.4 \\
1.7 \\
90.5\end{array}$ & $\begin{array}{r}150.7 \\
55.6 \\
1.8 \\
95.1\end{array}$ & $\begin{array}{r}157.8 \\
55.1 \\
1.7 \\
102.7\end{array}$ \\
\hline \multicolumn{14}{|l|}{ Memorandum Items } \\
\hline $\begin{array}{l}\text { Avg daily reserve money in 3rd month of quarter } \\
12 \text {-month percent change } \\
\text { Avg daily currency in 3rd month of quarter } \\
\text { 12-month percent change }\end{array}$ & $\begin{array}{l}23.6 \\
28.5 \\
15.7\end{array}$ & $\begin{array}{l}22.0 \\
25.8 \\
13.8 \\
32.8\end{array}$ & $\begin{array}{l}22.8 \\
30.4 \\
14.3 \\
38.2\end{array}$ & $\begin{array}{l}24.6 \\
23.7 \\
15.3 \\
25.7\end{array}$ & $\begin{array}{l}25.4 \\
27.9 \\
16.1 \\
31.9\end{array}$ & $\begin{array}{l}25.8 \\
22.1 \\
16.1 \\
17.4\end{array}$ & $\begin{array}{l}28.9 \\
37.0 \\
17.9 \\
30.7\end{array}$ & $\begin{array}{l}29.5 \\
25.0 \\
18.6 \\
18.7\end{array}$ & $\begin{array}{l}31.8 \\
35.0 \\
20.4 \\
30.1\end{array}$ & $\begin{array}{l}27.4 \\
19.9 \\
15.9 \\
10.9\end{array}$ & $\begin{array}{l}29.8 \\
17.4 \\
18.4 \\
14.6\end{array}$ & $\begin{array}{l}33.7 \\
16.6 \\
21.9 \\
22.6\end{array}$ & $\begin{array}{l}36.4 \\
14.3 \\
24.1 \\
18.1\end{array}$ \\
\hline NCG stock (prog def.) ${ }^{2}$ & -14.6 & -11.3 & -12.1 & -15.7 & -15.9 & -13.1 & -17.8 & -13.6 & -16.3 & -12.5 & -17.2 & -16.7 & -14.3 \\
\hline NCG flow (prog def.) cum from end-year ${ }^{2}$ & 0.5 & 2.6 & 2.5 & -1.7 & -1.3 & 0.9 & -3.2 & 1.0 & -1.7 & 1.1 & -0.9 & -0.4 & 2.1 \\
\hline $\begin{array}{l}\text { 12-month percent change } \\
\text { Reserve money } \\
\text { M2 } \\
\text { M3 } \\
\text { Credit to the economy }\end{array}$ & $\begin{array}{l}27.3 \\
34.6 \\
32.6 \\
58.6\end{array}$ & $\begin{array}{l}25.8 \\
32.9 \\
29.8 \\
47.9\end{array}$ & $\begin{array}{l}28.4 \\
32.6 \\
31.6 \\
50.5\end{array}$ & $\begin{array}{l}23.7 \\
33.7 \\
33.7 \\
60.0\end{array}$ & $\begin{array}{l}25.2 \\
28.1 \\
32.9 \\
56.5\end{array}$ & $\begin{array}{l}22.1 \\
27.3 \\
23.9 \\
27.5\end{array}$ & $\begin{array}{l}39.1 \\
24.7 \\
32.7 \\
48.6\end{array}$ & $\begin{array}{l}25.0 \\
25.4 \\
27.0 \\
36.0\end{array}$ & $\begin{array}{l}29.2 \\
17.6 \\
22.8 \\
27.5\end{array}$ & $\begin{array}{l}19.9 \\
27.7 \\
23.6 \\
33.7\end{array}$ & $\begin{array}{l}17.4 \\
18.7 \\
21.5 \\
26.2\end{array}$ & $\begin{array}{l}16.6 \\
19.7 \\
17.8 \\
19.6\end{array}$ & $\begin{array}{l}14.3 \\
20.4 \\
20.0 \\
19.5\end{array}$ \\
\hline $\begin{array}{l}\text { Money multiplier (M2/reserve money) } \\
\text { Velocity (GDP/M2) }\end{array}$ & $\begin{array}{l}2.97 \\
3.71\end{array}$ & $\begin{array}{l}3.17 \\
4.30\end{array}$ & $\begin{array}{l}3.10 \\
4.55\end{array}$ & $\begin{array}{l}3.09 \\
3.85\end{array}$ & $\begin{array}{l}2.93 \\
4.24\end{array}$ & $\begin{array}{l}3.16 \\
3.77\end{array}$ & $\begin{array}{l}2.71 \\
4.07\end{array}$ & $\begin{array}{l}2.97 \\
3.58\end{array}$ & $\begin{array}{l}2.70 \\
3.79\end{array}$ & $\begin{array}{r}3.30 \\
\ldots\end{array}$ & $\begin{array}{r}2.96 \\
\ldots\end{array}$ & $\begin{array}{r}2.78 \\
\ldots\end{array}$ & $\begin{array}{l}2.84 \\
3.70\end{array}$ \\
\hline $\begin{array}{l}\text { Nominal GDP } \\
\text { Nominal GDP growth }\end{array}$ & $\begin{array}{r}269 \\
12.1\end{array}$ & $\begin{array}{r}306 \\
16.3\end{array}$ & $\begin{array}{r}323 \\
20.1\end{array}$ & $\begin{array}{r}306 \\
16.3\end{array}$ & $\begin{array}{r}323 \\
20.1\end{array}$ & $\begin{array}{r}306 \\
16.3\end{array}$ & $\begin{array}{r}323 \\
20.1\end{array}$ & $\begin{array}{r}326 \\
20.9\end{array}$ & $\begin{array}{r}323 \\
20.1\end{array}$ & $\begin{array}{l}\cdots \\
\cdots\end{array}$ & $\begin{array}{l}\cdots \\
\cdots\end{array}$ & $\begin{array}{l}\cdots \\
\cdots\end{array}$ & $\begin{array}{r}380 \\
17.4\end{array}$ \\
\hline
\end{tabular}

Sources: Bank of Mozambique; and IMF staff estimates and projections.

${ }^{1}$ Includes disbursements of Fund resources under the ESF and the August 2009 SDR allocation.

${ }^{2}$ As defined in the TMU, this equals the NCG in the monetary survey excluding Moatize deposits, earmarked donor funds, and bonds issued to capitalize the BM. 
Table 5. Mozambique: Balance of Payments, 2009-16

(Millions of U.S. dollars, unless otherwise specified)

\begin{tabular}{|c|c|c|c|c|c|c|c|c|c|c|}
\hline & \multirow{2}{*}{$\begin{array}{c}2009 \\
\text { Act. }\end{array}$} & \multicolumn{2}{|l|}{2010} & \multicolumn{2}{|l|}{2011} & 2012 & 2013 & 2014 & 2015 & 2016 \\
\hline & & CR No. 10/375 & Est. & CR No. $10 / 375$ & Proj. & \multicolumn{5}{|c|}{ Projections } \\
\hline Current account balance & $-1,220$ & $-1,308$ & -999 & $-1,258$ & $-1,380$ & $-1,478$ & $-1,637$ & $-1,770$ & $-1,908$ & $-2,077$ \\
\hline Trade balance for goods & $-1,275$ & $-1,492$ & $-1,092$ & $-1,170$ & $-1,296$ & $-1,270$ & $-1,339$ & $-1,408$ & $-1,484$ & $-1,584$ \\
\hline Of which: Megaprojects & 520 & 765 & 768 & 1,113 & 925 & 1,287 & 1,515 & 1,768 & 2,053 & 2,355 \\
\hline Exports, f.o.b. & 2,147 & 2,328 & 2,243 & 2,804 & 2,574 & 3,020 & 3,350 & 3,702 & 4,092 & 4,500 \\
\hline Of which: Megaproject exports & 1,311 & 1,648 & 1,668 & 2,107 & 1,960 & 2,384 & 2,683 & 3,000 & 3,352 & 3,720 \\
\hline Imports, f.o.b. & $-3,422$ & $-3,819$ & $-3,335$ & $-3,974$ & $-3,871$ & $-4,289$ & $-4,689$ & $-5,110$ & $-5,575$ & $-6,084$ \\
\hline Of which: Megaproject imports & -791 & -883 & -900 & -993 & $-1,035$ & $-1,098$ & $-1,168$ & $-1,231$ & $-1,299$ & $-1,364$ \\
\hline Trade balance for services & -457 & -452 & -493 & -493 & -615 & -688 & -795 & -873 & -949 & $-1,041$ \\
\hline Income balance & -251 & -169 & -85 & -514 & -225 & -329 & -384 & -439 & -496 & -512 \\
\hline Of which: Dividend payments by megaprojects & -17 & -222 & 0 & -461 & -219 & -296 & -326 & -357 & -397 & -399 \\
\hline Current transfers balance & 763 & 805 & 671 & 920 & 757 & 809 & 881 & 950 & 1,022 & 1,060 \\
\hline Of which: External grants & 687 & 716 & 605 & 824 & 675 & 718 & 780 & 839 & 901 & 927 \\
\hline Capital and financial account balance & 1,288 & 1,151 & 1,208 & 1,477 & 1,471 & 1,780 & 1,895 & 2,138 & 2,324 & 2,535 \\
\hline Capital account balance & 422 & 349 & 349 & 375 & 431 & 482 & 535 & 588 & 640 & 739 \\
\hline Financial account balance & 865 & 802 & 859 & 1,103 & 1,040 & 1,298 & 1,360 & 1,550 & 1,684 & 1,796 \\
\hline Net foreign direct investment & 890 & 808 & 790 & 923 & 867 & 958 & 980 & 1,154 & 1,209 & 1,344 \\
\hline Net foreign borrowing by the general government & 434 & 567 & 476 & 861 & 885 & 974 & 993 & 947 & 885 & 854 \\
\hline Net foreign borrowing by the nonfinancial private sector & -487 & -305 & -348 & -191 & -343 & -338 & -314 & -307 & -215 & -209 \\
\hline Other 1 & 28 & -268 & -59 & -490 & -368 & -296 & -299 & -243 & -195 & -194 \\
\hline Of which: Special drawing rights & 170 & 0 & 0 & 0 & 0 & 0 & 0 & 0 & 0 & 0 \\
\hline Net errors and omissions & 126 & 0 & -151 & 0 & 0 & 0 & 0 & 0 & 0 & 0 \\
\hline Overall balance & 194 & -157 & 58 & 220 & 91 & 302 & 259 & 368 & 416 & 458 \\
\hline External financing & -194 & 157 & -58 & -220 & -91 & -302 & -259 & -368 & -416 & -458 \\
\hline Reserve assets 1 & -352 & 136 & -87 & -218 & -89 & -299 & -256 & -335 & -355 & -391 \\
\hline Net use of Fund credit & 156 & 21 & 18 & -2 & -2 & -3 & -3 & -34 & -61 & -67 \\
\hline Exceptional financing & 2 & 0 & 10 & 0 & 0 & 0 & 0 & 0 & 0 & 0 \\
\hline \multicolumn{11}{|l|}{ Memorandum items: } \\
\hline \multicolumn{11}{|l|}{ Effective exchange rate indexes (percent change) } \\
\hline Nominal effective exchange rate & -7.1 & $\ldots$ & -22.4 & $\ldots$ & $\ldots$ & $\ldots$ & $\ldots$ & $\ldots$ & $\ldots$ & $\ldots$ \\
\hline Real effective exchange rate & -6.3 & $\ldots$ & -15.0 & $\ldots$ & $\ldots$ & $\ldots$ & $\ldots$ & $\ldots$ & $\ldots$ & $\ldots$ \\
\hline Terms of trade index (percent change) & -0.7 & 2.5 & 15.0 & 13.1 & 4.9 & 5.2 & -0.3 & -3.4 & -1.8 & 1.2 \\
\hline International aluminum price & -35.3 & 24.2 & 30.2 & 1.6 & 19.6 & 3.5 & 0.9 & 0.6 & 1.8 & 0.0 \\
\hline International food price index & -14.7 & 6.8 & 11.4 & -1.3 & 24.1 & -4.7 & -6.4 & -5.4 & -3.8 & -1.5 \\
\hline International fuel price index & -36.9 & 21.9 & 26.0 & 3.1 & 31.9 & 0.1 & -2.2 & -1.0 & 0.3 & 0.9 \\
\hline Current account balance (percent of GDP) & -12.1 & -13.4 & -10.2 & -12.0 & -11.4 & -10.9 & -10.9 & -10.7 & -10.6 & -10.5 \\
\hline Excluding external grants & -18.9 & -20.7 & -16.4 & -19.8 & -17.0 & -16.2 & -16.1 & -15.8 & -15.6 & -15.2 \\
\hline Gross aid inflows (percent of GDP) & 15.6 & 15.2 & 13.4 & 15.0 & 12.4 & 11.9 & 11.7 & 11.5 & 11.4 & 11.2 \\
\hline Of which: To central government & 15.0 & 14.2 & 12.7 & 13.6 & 11.4 & 11.0 & 11.1 & 11.0 & 11.0 & 11.1 \\
\hline Budget support & 4.9 & 5.1 & 4.9 & 4.3 & 3.7 & 3.6 & 3.6 & 3.5 & 3.5 & 3.5 \\
\hline Nonbudge support & 10.1 & 9.1 & 7.8 & 9.3 & 7.7 & 7.4 & 7.5 & 7.5 & 7.5 & 7.6 \\
\hline Project support & 8.6 & 8.8 & 6.7 & 8.2 & 6.7 & 6.4 & 6.6 & 6.6 & 6.6 & 6.6 \\
\hline Onlending & 1.5 & 0.4 & 1.1 & 1.1 & 1.0 & 1.0 & 0.9 & 0.9 & 0.9 & 0.9 \\
\hline Net foreign assets & 1,701 & 1,544 & 1,745 & 1,763 & 1,836 & 2,138 & 2,397 & 2,765 & 3,182 & 3,639 \\
\hline Net international reserves 1 & 1,840 & 1,675 & 1,908 & 1,895 & 2,000 & 2,302 & 2,560 & 2,928 & 3,345 & 3,802 \\
\hline Gross international reserves 1 & 2,012 & 1,868 & 2,099 & 2,086 & 2,189 & 2,487 & 2,743 & 3,077 & 3,432 & 3,823 \\
\hline Months of projected imports of goods and nonfactor services & 5.4 & 4.4 & 4.8 & 4.5 & 4.5 & 4.7 & 4.7 & 4.8 & 4.9 & 5.0 \\
\hline Months of current imports of goods and nonfactor services & 5.4 & 4.6 & 5.6 & 4.9 & 5.0 & 5.1 & 5.1 & 5.3 & 5.4 & 5.5 \\
\hline
\end{tabular}

Sources: Data from Government of Mozambique and projections by IMF staff.

${ }^{1}$ Accounts for disbursement of an SDR allocation of SDR 108.8 million in 2009Q3 above the line under other investment liabilities of the monetary authorities, and below the line as an

increase in reserve assets. 


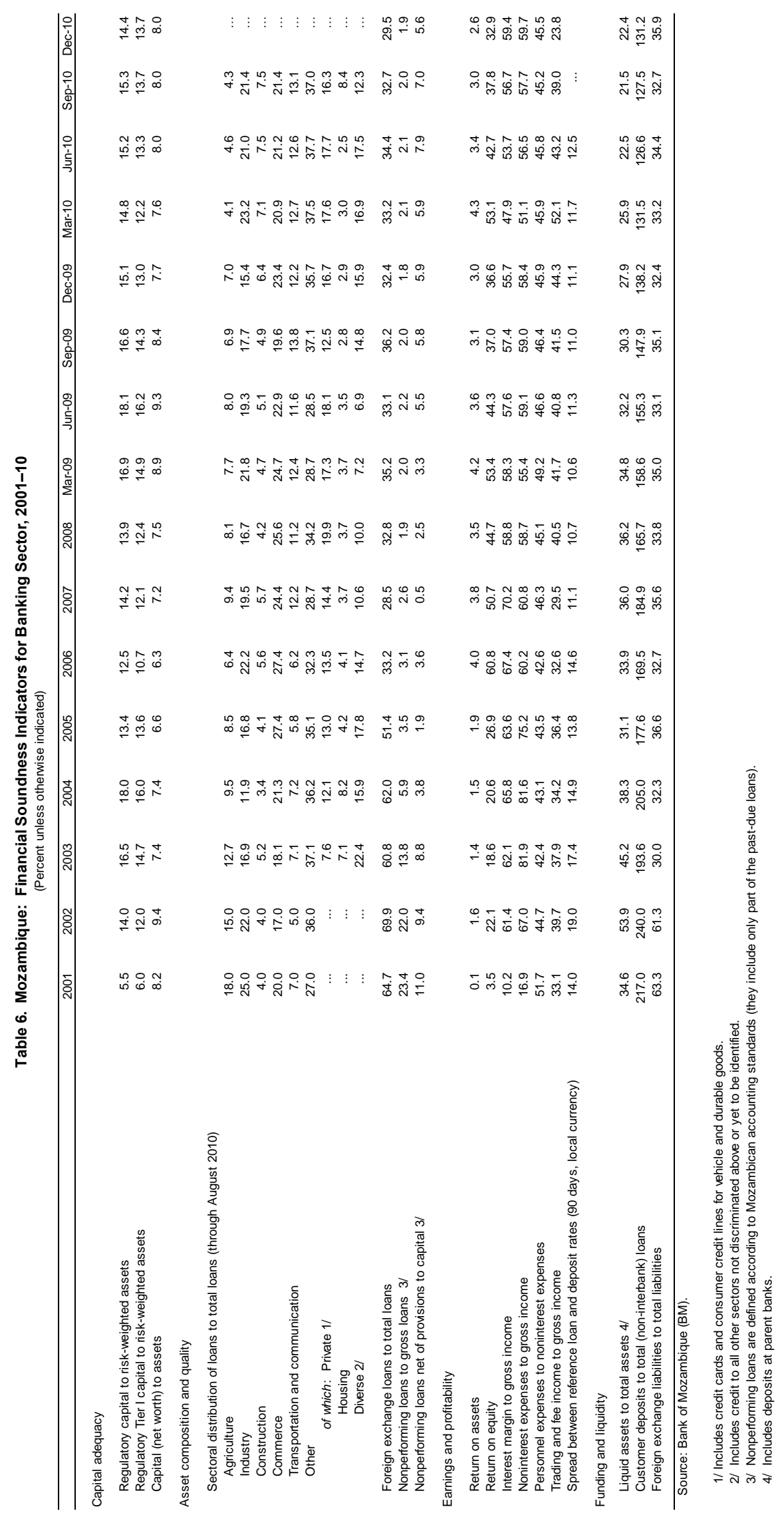


Table 7. Mozambique: Structural Conditionality under the current PSI, 2010-2011

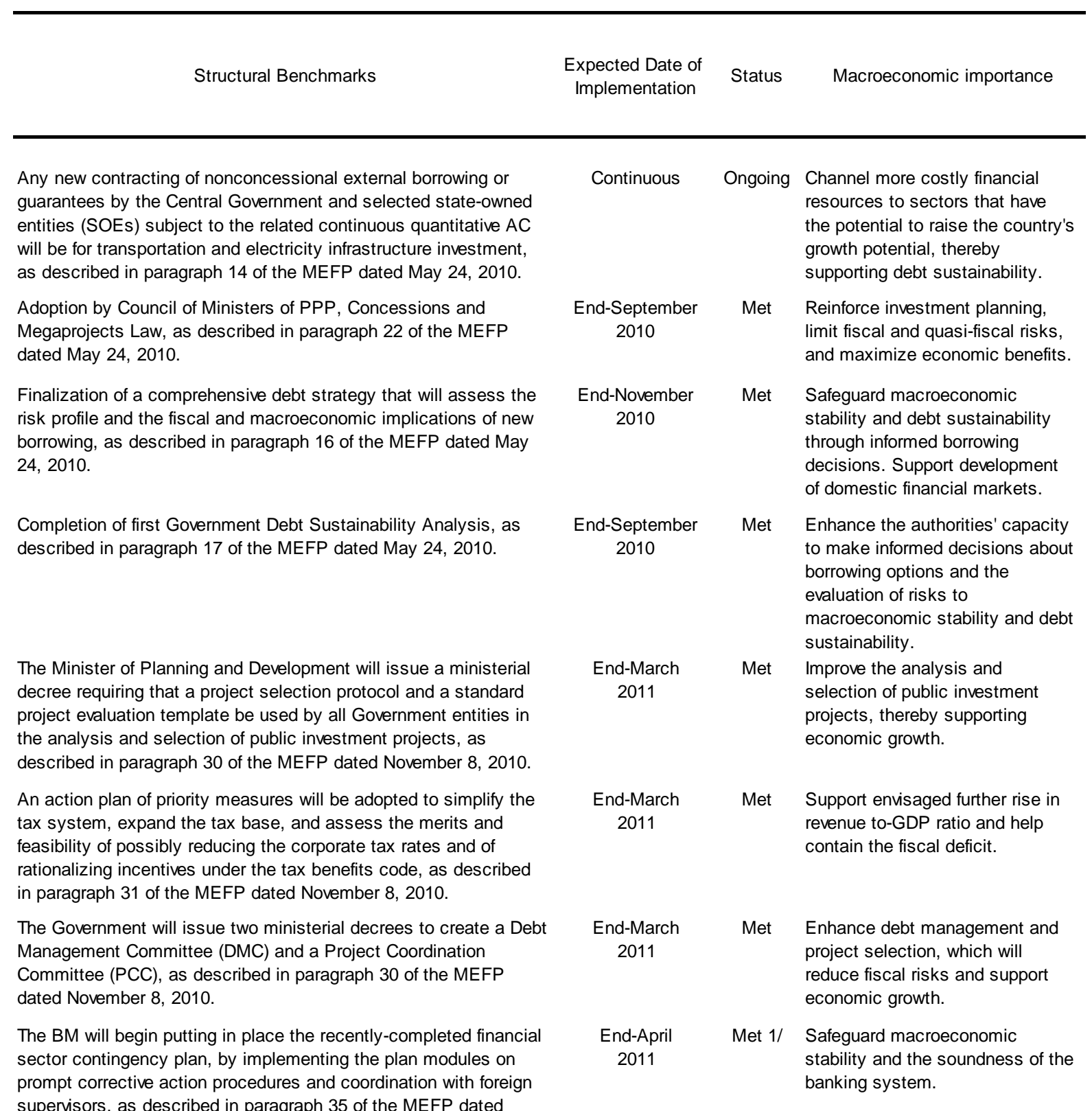
November 8, 2010.

1/ As of May 17, 2011 the measure has largely been complemented. What is outstanding is the signature of a Memorandum of Understanding with the South African bank supervisors to formalize the cooperation and information sharing agreement with the Mozambican authorities. This is expected to be signed soon. 


\section{APPENDIX I: LETTER OF INTENT-MOZAMBIQUE}

\section{Mr. John Lipsky \\ Acting Managing Director \\ International Monetary Fund \\ Washington, D.C. 20431 \\ U.S.A.}

May 20, 2011

\section{Dear Mr. Lipsky:}

The Government of Mozambique requests the completion of the second review under the Policy Support Instrument (PSI). In support of this request, we are transmitting the attached Memorandum of Economic and Financial Policies (MEFP), which reviews implementation of our economic program under the PSI and sets out the Government's objectives and policies over the short and medium term.

The Government's economic program aims to maintain macroeconomic stability, promote more inclusive growth through productive employment, and reduce poverty. The program is consistent with the new Poverty Reduction Strategy Paper (PARP) for 2011-14, which the Government formally adopted on May 3, 2011.

We met all quantitative assessment criteria (AC) through end-December 2010, except for the one on reserve money. We also made strong progress in implementing our structural reform program.

The Government believes that the policies outlined in the MEFP are adequate to achieve the objectives of the PSI-supported program. We stand ready to take any additional measures necessary to achieve our policy objectives. The Government will consult with the IMF-at its own initiative or whenever the Managing Director requests such a consultation-should revisions be contemplated regarding the policies contained in the attached MEFP. The Government will provide the IMF with such information as the IMF may request to be able to assess the progress made in implementing the economic and financial policies and achieving the objectives of the program.

Sincerely yours,

$\begin{array}{cc}\text { /s/ } & / \mathrm{s} / \\ \text { Manuel Chang } & \text { Ernesto Gouveia Gove } \\ \text { Minister of Finance } & \text { Governor } \\ & \text { Bank of Mozambique }\end{array}$

Attachments: Memorandum of Economic and Financial Policies

Technical Memorandum of Understanding 


\section{Attachment 1: Memorandum of Economic and Financial Policies} May 20, 2011

1. This updated MEFP (i) describes recent developments and performance under the Government of Mozambique's economic program under the three-year Policy Support Instrument (PSI) through April 2011; (ii) summarizes the Government's poverty reduction strategy envisaged in the new PARP; and (iii) elaborates on macroeconomic policy and structural reform intentions for 2011. It builds on the MEFPs underlying Mozambique's PSI request and first review dated May 28, 2010 and November 8, 2010, respectively.

\section{RECENT ECONOMIC DEVELOPMENTS AND PERFORMANCE UNDER THE PROGRAM}

\section{Mozambique continues to show resilience in a turbulent external economic} environment. Our economy has weathered the global financial crisis well. Real GDP growth in 2010, at an estimated 6.6 percent, fell slightly below expectations but was one of the highest in the region. Economic activity was broad-based and supported by strong global demand. Higher megaproject exports offset the rising import bill related to surging fuel and food prices and helped keep the current account deficit well below expectations. However, the recent increases in food and fuel prices, through their secondary effects, prevented core inflation from reversing its upward trend, despite our determined efforts to tighten monetary and fiscal policies. Remarkably strong revenue collections were instrumental in containing the primary domestic fiscal deficit to $31 / 4$ percent of GDP, some 1 percent of GDP lower than programmed, notwithstanding higher outlays for the fuel subsidy and emergency spending to mitigate the impact of higher food prices on the poor.

\section{Amidst the difficult environment, Mozambique's program performance remains} strong. All end-2010 quantitative AC have been met, except for reserve money. Quarterly reserve money growth decelerated from 121/2 percent in Q3 to 73/4 percent in Q4, above the targeted Q4 growth rate of 5 percent. This was due, inter alia, to bunched Government spending in mid-December 2010. Relative to prior years, this unusual spending pattern reflected the delayed beginning of budget execution, since the 2010 budget was approved only in May 2010 due to the formation of the new Government following the elections. This in turn left the Bank of Mozambique (BM) with little time to counter the resulting monetary expansion. With respect to the indicative target on priority spending, the Government executed the domestic investment component in full, but fell short by MT 2.2 billion in domestic current spending necessitated by the higher costs of the fuel subsidy. In addition, the externally financed component fell short of the amounts included in the 2010 budget law, mainly since investment projects in infrastructure and agriculture experienced delays. The ACs on net international reserves (NIR) and net credit to the Government (NCG) were comfortably met. As to non-concessional borrowing (NCB), we signed a nonconcessional loan from China for revamping Maputo airport in the amount of US\$66 million in December 2010, and from Brazil to rehabilitate Nacala airport in the amount of US\$ 80 million in April 2011. 
4. Structural reforms are on track as well. All structural benchmarks through endApril have been met. However, the end-November 2010 benchmark on developing a medium-term debt strategy (MTDS) was completed with a five-month delay, since the conceptual work required more time. We have decided to continue working on the strategy and revisit it later this year, once our enhancements in debt management and project selection have been properly implemented and we have benefited from additional technical assistance. We will submit a refined MTDS to the Council of Ministers for approval by mid-December 2011. With respect to the financial sector contingency plan, we have undertaken all necessary steps under our control and are, as a final step, awaiting signature of a Memorandum of Understanding with the South African bank supervisors to formalize the cooperation and information sharing agreement.

\section{Poverty Reduction Strategy}

5. The new four-year PARP adopted by the Council of Ministers on May 3, 2011 demonstrates the Government's commitment to more inclusive growth. It aims at reducing poverty from 54.7 percent in 2009 to 42 percent by 2014 and rests on three main pillars: (i) the increase in production and productivity in the labor-intensive sectors of agriculture and fisheries; (ii) job creation through improvements in the business environment; and (iii) human and social development, including through the significant expansion of social protection systems. It will be embedded in two supporting pillars: (i) the maintenance of a sound macroeconomic framework; and (ii) our strong commitment to good governance.

6. The Government designed the PARP as a dynamic and flexible document. Its objectives and indicators will be reviewed and, if needed, updated annually under the Economic and Social Plan (PES) in light of national and international developments and in tandem with the implementation of sectoral strategies, such as the forthcoming Agricultural Reform Strategy, the Financial Sector Development Strategy, and the successor program to the 2008-12 Strategy for the Improvement of the Business Environment in Mozambique. In all of those efforts, the Government will pursue a coordinated approach across ministries, recognizing the interlinkages across policy areas and institutions.

\section{The poverty reduction strategy complements the Government's overall} approach to economic development. The PARP's strong focus on fostering social cohesion will be conducive to supporting the Government's objective of promoting long-term economic growth and complement the Government's intentions to further exploit Mozambique's rich endowment of natural resources and rapidly developing public infrastructure. In this context, the Government will continuously seek to create fiscal space, including by improving the management of revenues from the natural resources sector so as to help finance the closing of the infrastructure and skills gaps and the expansion of social safety nets.

8. The PARP aims at building sustainable social safety nets to help reduce poverty while strengthening Mozambique's long-term growth potential. It envisages a comprehensive stocktaking of current social protection mechanisms to be carried under our 
National Basic Social Security Strategy (ENSSB) and the Strategic Program for the Reduction of Urban Poverty (PERPU). Several key undertakings are planned for the first half of 2011 to support the evolving national policy dialogue, culminating in a Council of Ministers meeting in July 2011 where key decisions regarding the future direction of basic social security will be taken. These decisions will be informed by ongoing exercises, supported by various development partners, such as a Public Expenditure Review in social protection and a costing exercise around the different expansion options.

\section{Macroeconomic Policies}

\section{A. Economic Objectives}

9. In spite of the challenging international environment, the Government foresees a positive macroeconomic outlook for 2011. Economic growth could reach $71 / 4$ percent in 2011 and over the medium term. Our strong efforts to arrest inflation expectations are expected to decelerate headline inflation to single digits by end-2011, subject to international food and fuel price developments, and to the Authorities' inflation objective of 6 percent over the medium term. The external current account deficit (after grants) is set to stabilize between 10 and 11 percent of GDP during the next few years, while the reserves coverage of imports should approach 5 months on account of increasing private capital inflows, exports from new megaprojects in the mining sector, and continued donor support.

\section{B. Macroeconomic Policy Mix}

10. Arresting inflation expectations is the immediate priority for 2011. The BM will sharply decelerate monetary expansion to unwind the monetary stimulus adopted in the wake of the global crisis and to rein in second-round spillovers from international to domestic prices. Fiscal policy will be supportive to the BM's efforts, with a further reduction of the domestic primary deficit and limited recourse to domestic financing which should also help avoid a crowding out of the private sector.

\section{Over the medium-term, consistent with the PARP, we will adhere to a policy} stance that supports economic development and allows more Mozambicans to directly benefit from economic activity. These efforts will be embedded in a policy framework that preserves macroeconomic stability and debt sustainability, consistent with our strong track record in this area. The envisaged policy stance will aim to be sufficiently tight to allow the BM to achieve its inflation objectives. At the same time, it envisages stepping up investment spending to close the skills and infrastructure gaps, which necessitates a temporary-albeit smaller-than-previously expected - increase of the overall fiscal deficit to about 6 percent of GDP during the program period. This increase partly reflects nonconcessional external borrowing for investment projects that are expected to have a high economic return. Part of the stepped-up investment program, but also of the intended expansion of social safety nets, will also be financed by a continued strong revenue effort. As a result, we expect to be able to stabilize the domestic primary deficit—our fiscal anchor-at around 3 to $3 \frac{1}{2}$ percent of GDP during the period. 


\section{Monetary and Exchange Rate Policies}

\section{The Authorities are determined to bringing inflation back to single digits from}

2011 onwards. It is our objective to contain average inflation to $9 \frac{1}{2} 2$ percent in 2011 and below 6 percent over the medium term. To this end, the BM will aim to decisively slow down monetary expansion in the near term, building on our achievements in 2010 when broad money (M3) growth decelerated by one-third and private sector credit growth by more than half relative to the year before. We attribute this to the sharp increases in our key policy rate, stepped-up foreign exchange and Treasury bill (BT) interventions, and, in particular, the increased reserve requirements that effectively curtailed commercial banks' liquidity and ability to extend loans.

13. We will curtail reserve money growth to around 14 percent in 2011 , after 29 percent in 2010. In this context, the BM will carefully monitor reserve money developments so as to keep its main operational target on a trajectory that allows meeting the quarterly program targets. In the process, it will rely on its full set of monetary policy instruments to drain liquidity. Should second-round inflation pressures persist, the BM stands ready to further tighten its monetary policy as needed.

14. The BM intends to further strengthen its monetary policy operations framework in line with technical assistance recommendations. This will be conducive to preparing its move to an inflation targeting framework over time, improving open market operations, developing financial markets, and enhancing the coordination with the government to facilitate liquidity forecasting. Specifically:

- Improving liquidity forecasting: The BM and the government will implement the Memorandum of Understanding (MoU) signed on March 25, 2011 on the exchange of information.

- $\quad$ Consolidating monetary policy instruments: By end-October 2011, the BM will reduce the frequency of BT issues, concentrate the BT maturities, and make them fungible in the interbank money market (MMI) module. The BM will consider in due course publishing a BT issuance calendar providing information on auction frequency for each maturity.

15. Mozambique's flexible exchange rate regime has served the country well and remains appropriate. The $\mathrm{BM}$ will allow the exchange rate to adjust freely to evolving patterns of trade and financial flows while further strengthening Mozambique's international reserves, which are projected to approach 5 months of projected imports over the medium term. Following the strong depreciation in 2010, current indications point toward the exchange rate being broadly in line with fundamentals. The BM will continue to closely monitor developments in the real effective exchange rate vis-à-vis a broad basket of currencies. 
16. On May 20, 2011, we notified the Fund of our acceptance of the obligations under Article VIII sections 2, 3, and 4 of the Fund's Articles of Agreement. New foreign exchange regulations were issued on December 31, 2010. Consistent with recent Fund technical assistance recommendations, the BM will issue shortly implementing guidelines which provide further clarifications on procedural aspects of the foreign exchange regulations so as to ensure that all previously existing exchange restrictions have effectively been removed. On March 30, 2011, the BM adopted formal decisions to (i) limit potential deviations between the exchange rates in the interbank market (MCI) and the exchange rate used by the BM in its transactions with the government and the World Bank; and (ii) temporarily discontinue its multiple price auction system which in any case had not been used since 2009. The latter decision has been communicated to banks in writing and will remain in effect until further notice.

\section{Fiscal Policy}

17. Fiscal policy will aim to enable the Government's development strategy, but in the short run be geared toward supporting the BM's monetary tightening to fight inflation. To that effect, we will aim to limit the primary domestic fiscal deficit to 3 percent of GDP in 2011 , close to $1 / 4$ percent of GDP lower than initially projected. This entails that NCG will be contained to $1 / 2$ percent of GDP. The lower deficit target reflects prudence in expenditure execution and continued buoyancy in revenue collections, which we expect to reach MT 76.8 billion this year (20.2 percent of GDP), some MT 5 billion higher than the projections underlying the November 2010 MEFP. The program's indicative revenue floor is prudently set $1 / 2$ percent of GDP below the ambitious objectives of the revised 2011 budget law, but the Revenue Authority will be instructed to collect the full revenue target under the revised budget law (20.7 percent of GDP).

18. We have submitted a revised budget to parliament to reallocate expenditure appropriations in line with changing spending priorities. In particular, we will increase the budgetary ceiling for transfers and subsidies so as to accommodate the costs of past fuel subsidies and new government programs to subsidize the consumption of food products and urban transportation in light of continued high world prices. Consistent with the organic budget law (SISTAFE 2002), an amendment in the planned revised budget law will exceptionally authorize the government to reinforce outlays in the subsidies and transfers budget category from contingency lines in other budgetary categories should adverse circumstances arise.

19. The revenue contingency instituted on April 1, 2011 through administrative acts-Delegação de Competência sobre a Execução do Orcamento No. 4/2011 and Circular Ministerial No. 01/GM-MF de 05/01/2011 - remains in force. Consistent with SISTAFE 2002, they limit budget execution to cautionary ceilings as long as revenue collections remain uncertain. Accordingly, the following contingency ceilings relative to the planned revised budget apply: (i) 90 percent for goods and services and other current expenditures; (ii) 85 percent for civil service wages and transfers; and (iii) 90 percent for domestically-financed capital spending. 
20. We consider the higher budgetary costs of previously adopted support measures for fuel and food products as temporary and work toward their elimination. Over the medium term, we intend to replace those measures by better targeted and fiscally sustainable social protection measures that will benefit an increasingly large number of our citizens (see Section II). To effectively contain the costs of the current subsidies, we will take the following measures:

- Gradually phasing out fuel subsidy during 2011: Following the subsidy removal for mega projects and several groups of wholesale customers, the Government is determined to gradually return to formula-based fuel price adjustments, provided that our planned better-targeted support mechanisms for the more vulnerable segments of the population can be implemented as planned (see below). It will seek an agreement with fuel importers to compensate them for any interim losses that may be incurred, considering various compensation options. Consistent with the agreement reached with fuel importers in December 2010, the Government will settle any debt incurred by fuel importers in 2010 by end-May 2011. Finally, it will continue to show the full costs of any government outlays for fuel products in budget documents, in line with best fiscal transparency principles.

- $\quad$ Refining food and transport subsidies for the poor: The Government intends to move away from generalized subsidies and design more targeted schemes. We plan to establish from August 2011 onwards a transport voucher covering low-income workers, students, and the elderly, and as of June 2011 a food voucher for eligible citizens, covering basic food staples in an effort to replace the current rice and bread subsidy. The Government will proceed with the ongoing efforts but also consider possible options that could ensure appropriate coverage.

21. Priority spending will be preserved despite the tight fiscal policy stance. Priority spending will, for the time being, continue to be defined as under the 2006-10 PARPA program categories, comprising spending in education, health, HIV/AIDS, infrastructure development, agriculture, rural development, governance, and the judicial system. Such spending is targeted from MT 49.2 billion in 2010 to MT 58 billion in 2011, allowing priority spending to account for at least half of all spending. Upon completion of the programming exercise later on in the year, priority spending will be redefined by capturing all spending related to the new PARP's three main pillars (agriculture, job creation, and social and human development) and supporting pillars (macroeconomic management and governance).

\section{Our investment priorities in transportation and energy infrastructure and} related program commitments, as specified in the November 2010 MEFP, remain valid. We will continue our prudent approach in selecting projects, especially those that will be financed with non-concessional resources. In our assessment, the temporary increase in the overall fiscal deficit over the next few years will remain consistent with debt sustainability. Our very gradual launch of the projects financed by those two external sources should be conducive to containing inflation pressures in the short run. 
23. The government is determined to proceed gradually and prudently with the implementation of the wage policy. The Government will assess in due course the findings of the recently completed review of the implementation strategy of the new salary policy. Current indications point to us being able to complete the enhancements to our electronic payroll reporting system (e-FOLHA) and our civil servant master database record (e-CAF) in line with the timeline specified in the November 2010 MEFP. The first monthly wage bill report will be issued by end-July 2011, providing an overview of the civil servant population, wage payments by sector and the impact of promotion and recruitment at all levels (structural benchmark). The system will be further rolled out to three provincial directorates by end-December 2011.

\section{Structural Reforms}

\section{A. Public Financial Management and Reporting}

24. Improving the reporting, monitoring, and control of budget execution remains a fiscal reform priority.

- $\quad$ Expanding budget coverage: The roll-out of the e-SISTAFE budget execution system will continue, with a view to completing it for all Government institutions over the next few years. To this effect, the Government aims to adopt by endSeptember 2011 a roll-out plan for the remaining budgetary entities that are currently not covered. To be cost effective, the Government will exclude entities from the rollout provided their spending and number of transactions fall below a threshold to be defined. By end-December 2011, e-SISTAFE will cover 45 percent of all spending and a total of 50 institutions.

- Integrating all public finance IT systems: By end-August 2011, we will adopt an IT master plan to support e-SISTAFE and other PFM components. It will provide a medium-term road map on IT priorities, define basic operating and hardware standards, and assess costs and risks associated with the expansion of electronic budget execution processes.

- $\quad$ Bringing state accounts in line with International Public Sector Accounting Standards (IPSAS): Based on recent technical assistance, the Accounting Directorate will undertake, by end-August 2011, an exercise to simulate IPSAS-compatible account data for the 2010 State Account report, in parallel with the current format. This will enhance accrual recording, integrate financial flows and stocks, and incorporate public entities into the general government budget. We will analyze the results of the exercise with a view to developing IPSAS-compliant national state account presentation in the coming years.

\section{B. Debt Management and Investment Planning}

25. We intend to maintain the reform momentum on debt management and investment planning and aim to make the newly created framework operational. As outlined above, our focus will be to launch the work of the new Debt Management 
Committee (DMC) and Project Coordination Committee (PCC) to help us improve our decision making in these areas.

- Launching DMC and PCC: The DMC, an advisory body to ensure prudent borrowing decisions, will meet quarterly, with its inaugural meeting scheduled for end-June. The work plan aims to allow final approval of the MTDS by the Council of Ministers by mid-December 2011. It also envisages that the DMC (i) establishes procedures to ensure timely and regular data provision; (ii) supports the finalization of the second Government Debt Sustainability Analysis (DSA), to be completed by end-December 2011; (iii) reviews and advises on the first annual borrowing plan for 2012 (see below); and (iv) seeks donor support to develop domestic financial markets from 2012 onwards.

- Implementing new project selection processes: To strengthen capacity to identify and implement high-potential investment projects, we will strictly implement the newly adopted project selection protocol and standard evaluation template. The DMC and PCC will each issue quarterly reports to the respective supervising minister, providing a full overview on proposed projects and their evaluation, as well as their implications for debt management. The first of these reports will be ready by endSeptember 2011.

- Developing annual borrowing plan: The Government will develop, upon approval of the budget law by parliament, an annual borrowing plan for all domestic borrowing envisaged for 2012. The plan will be consistent with the Government's projected net annual financing needs and the projected cash flow throughout the year.

- Identifying pipeline of investment projects: The Integrated Investment Program will be prepared, and approved by the Minister of Planning and Development by endDecember 2011, in consultation with the PCC (structural benchmark). The program will list the government's priority investment projects for the next four years, and be consistent with the broad strategic objectives outlined in the new PARP.

\section{Tax Policy and Administration}

26. The Government is committed to a more business-friendly environment, and simplifying our tax system is key to achieving this target. Based on the approved action plan of priority measures (a completed structural benchmark for end-March 2011), we will initially focus on further modernizing tax administration, particularly with the support of new technologies. This will include the continued development and roll-out of customs and tax IT systems (single window and e-Tax, respectively), which will generate strategic information to guide subsequent tax policy reforms.

\section{The Government will step up efforts to improve tax compliance through automated revenue collection and better monitoring of large taxpayers.}

- $\quad$ Facilitating tax payment through banks: By end-July 2011, the Ministry of Finance, in coordination with the BM, will approve the Strategic Plan for Tax Payments 
through Banks, defining a time-bound roll-out plan. This will allow real-time control over budgetary resources thanks to the prompt channeling of revenues to the single treasury account (CUT).

- $\quad$ Strengthening Large Taxpayers Division (LTD - DCAT): The LTD's coverage will be expanded from 47 percent at end-2009 to 70 percent by end-2014, expecting to reach 52 percent by the end-2011. To expand the coverage of LTD, we will integrate the revenue collected by the Customs Directorate from the large taxpayers. Moreover, in order to improve processes, enhance cooperation, and provide incentives for voluntary compliance of large taxpayers, we will start in 2011 with the implementation of the model of "Taxpayer Manager, " assigning (initially) a number of large taxpayers to one manager.

- $\quad$ Lowering transaction costs for taxpayers: The Government intends to reduce, and possibly eliminate, stamp tax obligations on administrative acts. A study to this effect will be completed by end-December 2011, on which basis a proposal will be formulated to take the legal reform forward in 2012.

\section{Improving Natural Resource Management}

28. We expect to become a full-fledged EITI member soon while we will continue to improve the transparency of natural resource revenues. In our view, we have completed in a timely manner all formal requirements to acquire the EITI membership status. The Government reiterates its commitment to the EITI objectives and work toward further improving the intersectoral exchange of information so as to assure that timely and adequate information is available on mega-projects in general, and on the extractive industry in particular. Following the first EITI audit report recommendations, we intend to (i) assess and, if necessary, improve procedures on the valuation of minerals; (ii) determine whether capital gains can be considered as source of revenue, in line with best international practice; and (iii) undertake a study to define a proper tax allowance regime that clearly differentiates between depreciation and capital allowances in the context of fiscal mining regulation, with technical assistance sought from the IMF.

29. The Government will consider options to enhance revenues from the natural resources sector. In tandem with the ongoing public debate, the Government will carefully analyze the perspectives for potential renegotiation of fiscal benefits in the natural resources sector with willing investors while safeguarding the stability of the business climate in Mozambique. With a more medium-term objective, supported by technical assistance that is being sought, the Government will assess the feasibility of creating a stabilization fund that could accumulate revenues from Mozambique's rich minerals endowment and channel them to targeted spending that would be smoothed over time and strengthen the country's longterm growth factors.

\section{E. Fiscal Decentralization}

30. We intend to gradually implement our fiscal decentralization strategy to enhance service delivery, improve the link between spending and social outcome, and 
support economic development. Our strategy aims to initially transfer larger budget proposal and execution responsibilities to decentralized levels of the central government (Districtos, Provincias) in areas such as education, health, regional infrastructure, and for jobcreating projects. At the same time, the legal framework instituting the municipalities (Autarchias) allows for the devolution of certain taxation and spending responsibilities to incorporated cities and towns, including in the areas of urban transportation, basic health services, primary education, and water management. After the encouraging experience in places such as Maputo and Beira, we aim to further transfer responsibilities to other interested municipalities while at the same time ensuring that there is minimum capacity to manage those responsibilities and ensure proper internal controls.

\section{F. Good Governance Framework}

31. The Government acknowledges the importance of ensuring adequate governance standards in promoting the business environment and relations with development partners. The Attorney General's Office approved the Strategic Plan for the Central Bureau for the Fight against Corruption (GCCC) in January 2011. By end-July 2011, the Government will submit to Parliament, a package of administrative reforms to support the fight against corruption, including: (i) the revision of anti-corruption law 6/2004; (ii) the revisions of laws 4/1990 and 7/1998 on the declaration of assets by government officials; (iii) the revision of law 22/2007 reforming the organic law of the public sector, in particular regarding the competencies of GCCC; and (iv) a new law to define and regulate conflict of interest in the public service (structural benchmark). The legislation aims to conform with the United Nations Convention on Anti-Corruption and ensure that the GCCC has legal competencies for accusation of corruption-related crimes. The Government has begun consulting with relevant stakeholders, such as civil society and the private and public sectors, on the objectives and specifics of the legislation.

\section{G. Reform of National Social Security Institute (INSS)}

\section{We will continue to focus on strengthening the INSS' finances and organization.}

INSS will implement the new investment strategy that will be approved by end-May 2011 and solely aim to protect the interests of the beneficiaries. By end-September 2011, we will publish the validated 2009 accounts. By end-2011, we will approve the actuarial study which will be prepared based on audited accounts of 2004-08, with technical assistance support from the ILO. INSS will continue to revamp its IT system that will support the reorganization of human resources management. In 2012, we will seek to enhance coverage of the selfemployed and professionals, by integrating our database with the taxpayer information maintained by the tax authorities.

\section{Financial Sector Policies}

33. Financial sector development and stability is an essential ingredient for more inclusive growth in Mozambique. Improving financial intermediation and access to credit while safeguarding the soundness of the banking system remains a top priority. To this end, 
benefitting from the February 2011 validation workshop with development partners, we will finalize, by end-September 2011, the 2011-20 Financial Sector Development Strategy which will include a wide-ranging and clearly sequenced action plan, covering ways to, among other things, enhance financial inclusion, competition, consumer protection, and financial literacy.

\section{We will continue to closely monitor the banking system to assure its continued} soundness. While commercial banks weathered the impact of the global crisis well and were able to provide enterprises with financial resources as external funding became more scarce, the high credit growth of the last two years and the close financial sector linkages with banks abroad require the BM's focused attention. We intend to pursue the following reform efforts:

- $\quad$ Finalizing financial sector contingency plan: The plan's remaining plan modules will be adopted by end-November 2011. They include modules for (i) closing and liquidating banks, (ii) managing bidding processes for private sector takeovers, (iii) bailouts (i.e., taking banks into temporary public ownership), and (iv) emergency liquidity assistance to promote rehabilitation of problem banks into viable institutions (structural benchmark). Adoption of the remaining four modules will make the full contingency plan effective.

- Moving toward Basel II standards: With a view to strengthening the risk-based approach to banking supervision, the BM will begin implementing the recently adopted timetable for the migration to Basel II by 2014. The focus in 2011 will be on training of staff.

- Introducing deposit insurance: In light of the approval by the Council of Ministers' Decree on Deposit Insurance in November 2010, as envisaged in the 2009 FSAP, the Government will seek to launch the system as soon as the necessary financing, including from external sources and the budget, will be secured. In that event, the Minister of Finance will approve the necessary regulations, and the Board members of the related Executive Committee will be appointed.

- Broadening electronic banking: As part of the plans to promote financial inclusion to unserved areas and assure the safety and efficiency of the national payment system, the BM and commercial banks are in the process of establishing the Sociedade Interbancária de Serviços (SIMO), an interbank company that will be responsible for processing all retail electronic transactions in a single platform in 2011 (pilot phase). SIMO will also provide other interbank services, such as electronic funds transfer and mobile banking.

- $\quad$ Fighting money laundering: While the Financial Intelligence Unit (GIFIM) has been made fully operational, the amendment of the AML-CFT law is still awaiting parliamentary approval. Upon its approval, the BM will issue guidelines that would allow Mozambique to move toward compliance with the 40+9 Recommendations of the FATF. In order to ensure a consistent application of the AML-CFT framework across the various regulators, the Government will establish, and appoint the members of, the National AML Task Force by end-September 2011. 


\section{PROGRAM MONITORING}

35. The modified quantitative AC and indicative targets for end-June 2011, the indicative targets for end-September 2011, and the AC and indicative targets for end-December 2011 are shown in Table 1. Table 2 lists the structural benchmarks for the second half of 2011. The third PSI review is expected to be completed by end- 2011 and the fourth PSI review by endJune 2012. 
Table 1. Mozambique: Quantitative Assessment Criteria and Indicative Targets ${ }^{1}$

(Millions of meticais, unless otherwise specified)

\begin{tabular}{|c|c|c|c|c|c|c|c|c|c|c|c|c|c|c|}
\hline & \multicolumn{9}{|c|}{2010} & \multicolumn{5}{|c|}{2011} \\
\hline & \multicolumn{3}{|c|}{$\begin{array}{c}\text { End-June } \\
\text { Assessment Criteria }\end{array}$} & \multicolumn{3}{|c|}{$\begin{array}{c}\text { End-Sept } \\
\text { Indicative Target }\end{array}$} & \multicolumn{3}{|c|}{$\begin{array}{c}\text { End-Dec } \\
\text { Assessment Criteria }\end{array}$} & \multirow{2}{*}{$\begin{array}{c}\begin{array}{c}\text { End-March } \\
\text { Indicative } \\
\text { Target }\end{array} \\
\text { Prog. }\end{array}$} & \multicolumn{2}{|c|}{$\begin{array}{l}\text { End-June } \\
\text { Assessment } \\
\text { Criteria }\end{array}$} & \multirow{2}{*}{$\begin{array}{c}\text { End-Sept } \\
\text { Assessment } \\
\text { Criteria } \\
\text { Proposed }\end{array}$} & \multirow{2}{*}{$\begin{array}{c}\text { End-Dec } \\
\text { Assessment } \\
\text { Criteria } \\
\text { Proposed }\end{array}$} \\
\hline & Prog. & Adjusted & Act. & Prog. & Adjusted & Act. & Prog. & Adjusted & Act. & & Prog. & Proposed & & \\
\hline \multicolumn{15}{|l|}{ Assessment/Performance Criteria for end-June/December } \\
\hline Net credit to the government (cumulative ceiling) & $-1,743$ & $-1,218$ & $-1,287$ & 868 & 868 & $-3,215$ & 1,039 & 1,069 & $-1,737$ & 1,097 & $-2,012$ & -875 & -362 & 2,086 \\
\hline Stock of reserve money (ceiling) & 24,567 & 25,067 & 25,402 & 25,753 & 26,253 & 28,900 & 29,493 & 30,243 & 31,849 & 27,368 & 29,156 & 29,826 & 33,708 & 36,418 \\
\hline Stock of net international reserves of the BM (floor, US\$ millions) & 1,759 & 1,716 & 1,742 & 1,885 & 1,891 & 1,781 & 1,675 & 1,667 & 1,908 & 1,715 & 1,765 & 1,835 & 1,905 & 2,000 \\
\hline Stock of short-term external public debt outstanding (ceiling) & 0 & 0 & 0 & 0 & 0 & 0 & 0 & 0 & 0 & 0 & 0 & 0 & 0 & 0 \\
\hline External payments arrears (ceiling) & 0 & 0 & 0 & 0 & 0 & 0 & 0 & 0 & 0 & 0 & 0 & 0 & 0 & 0 \\
\hline \multicolumn{15}{|l|}{ Indicative targets: } \\
\hline Government revenue (cumulative floor) & 27,049 & 27,049 & 27,943 & 41,732 & 41,732 & 44,306 & 59,940 & 59,940 & 63,489 & 14,929 & 34,311 & 36,609 & 56,382 & 76,792 \\
\hline Priority spending (cumulative floor) & $\ldots$ & $\ldots$ & $\ldots$ & 43,562 & 43,562 & 35,561 & 58,424 & 58,424 & 49,246 & 15,899 & 34,547 & 27,499 & 40,839 & 58,000 \\
\hline
\end{tabular}

Sources: Mozambican authorities and IMF staff estimates.

1 For definition and adjustors, see the Program Monitoring Section of the Memorandum of Economic and Financial Polices and the Technical Memorandum of Understanding. 
Table 2. Mozambique: Structural Benchmarks Under the PSI

\begin{tabular}{|l|c|}
\hline $\begin{array}{l}\text { Any new contracting of nonconcessional external borrowing or guarantees by the Central } \\
\text { Government and selected state-owned entities (SOEs) subject to the related continuous } \\
\text { quantitative AC (see Table 1) will be for transportation and electricity infrastructure } \\
\text { investment, as described in paragraph 14 of the MEFP dated May 24, 2010. }\end{array}$ & Continuous \\
\hline $\begin{array}{l}\text { Building on the enhancements to e-FOLHA and e-CAF, a first quarterly report will be issued } \\
\text { on the civil servant population, wage payments by sector, and the impact of promotion and } \\
\text { recruitment decision at all levels, as described in paragraph } 22 \text { of the MEFP dated } \\
\text { November 8, 2010. }\end{array}$ & End-July 2011 \\
\hline $\begin{array}{l}\text { The Bank of Mozambique will adopt the financial sector contingency plan's modules related } \\
\text { to (i) closing and liquidating banks, (ii) managing bidding processes for private sector } \\
\text { takeovers, (iii) bailouts, and (iv) emergency liquidity assistance to promote rehabilitation of } \\
\text { problem banks into viable institutions, as described in paragraph 34 of the current MEFP. }\end{array}$ & End-November 2011 \\
\hline $\begin{array}{l}\text { The Minister of Planning and Development will approve an Integrated Investment Program, in } \\
\text { consultation with the Project Coordination Committee, as described in paragraph 25 of the } \\
\text { current MEFP. }\end{array}$ & End-December 2011 \\
\hline $\begin{array}{l}\text { The Government will submit to Parliament, a package of administrative reforms to support the } \\
\text { fight against corruption, including: (i) the revision of anti-corruption law 6/2004; (ii) the } \\
\text { revisions of laws 4/1990 and 7/1998 on the declaration of assets by government officials; (iii) } \\
\text { the revision of law 22/2007 reforming the organic law of the public sector, in particular } \\
\text { regarding the competencies of the GCCC; and (iv) a new law to define and regulate conflict } \\
\text { of interest in the public service, as described in paragraph 31 of the current MEFP. }\end{array}$ & End-July 2011 \\
\hline
\end{tabular}




\section{AtTACHMEnt 2: TeChNiCAL MEMORANDUM OF Understanding}

May 20, 2011

1. This Technical Memorandum of Understanding (TMU) defines the quantitative assessment criteria, indicative targets, and structural benchmarks on the basis of which the implementation of the Fund-supported program under the Policy Support Instrument (PSI) will be monitored. In addition, the TMU establishes the terms and timeframe for transmitting the data that will enable Fund staff to monitor program implementation.

\section{DEFINITIONS}

\section{Net credit to the central government}

2. Net credit to the central government (NCG) by the banking system is defined as the difference between the outstanding amount of bank credits to the central government and the central government's deposits with the banking system, excluding deposits in project accounts with the banking system, recapitalization bonds issued to the Bank of Mozambique (BM), and proceeds from the signing fee for mineral resource exploration. Credits comprise bank loans, advances to the central government and holdings of central government securities and promissory notes. NCG will be calculated based on data from balance sheets of the monetary authority and commercial banks as per the monetary survey. The limits on the change in NCG by the banking system will be cumulative from end-December of the previous year.

3. The central government encompasses all institutions whose revenue and expenditure are included in the state budget (Orçamento do Estado): central government ministries, agencies, and the administration of 11 provinces. Although local governments (43 municipalities or autarquias) are not included in the definition because they are independent, the bulk of their revenue is registered in the state budget as transfers to local governments.

\section{Government revenue and financing}

4. Revenue is defined to include all receipts of the General Directorate of Tax (Direcção Geral dos Impostos, DGI), the General Directorate of Customs (Direcção Geral das Alfândegas, DGA), and nontax revenue, including certain own-generated revenues of districts and some line ministries, as defined in the budget. Net receipts from privatization received by the National Directorate of State Assets (Direcção Nacional do Património do Estado) and unrealized profits transferred by the central bank to the treasury will not be considered as revenue (above the line) and will be accounted for as other domestic financing (below the line).

5. For the purpose of program monitoring, revenue is considered as collected at the time when it is received by the relevant government collecting agencies, in cash or checks, or through transfers into the respective bank account.

\section{Priority social spending}

6. Priority social spending is based on the PARPA program categories. Accordingly, it will include total spending in the following sectors: (i) education; (ii) health; (iii) HIV/AIDS;

(iv) infrastructure development; (v) agriculture; (vi) rural development; and (vii) governance and judicial system. 


\section{Reserve money}

7. For the purposes of program monitoring reserve money is defined as the sum of currency issued by the BM and commercial banks' holdings at the BM. The target is defined in terms of the average of the daily end-of-day stocks in the month of the test date. The reserve money stock will be monitored and reported by the BM.

\section{Net international reserves}

8. Net international reserves (NIR) of the BM are defined as reserve assets minus reserve liabilities. The BM's reserve assets include (a) monetary gold; (b) holdings of SDRs; (c) reserve position at the IMF; (d) holdings of foreign exchange; and (e) claims on nonresidents, such as deposits abroad (excluding the central government's savings accounts related to mineral resource extraction concessions). Reserve assets exclude assets pledged or otherwise encumbered, including but not limited to assets used as collateral or guarantee for a third-party external liability (assets not readily available). The BM's reserve liabilities include: (a) all short-term foreign exchange liabilities to nonresidents with original maturity of up to and including one year; and (b) all liabilities to the IMF.

\section{New nonconcessional external debt contracted or guaranteed by the central government, the BM, and selected state-owned enterprises, with maturity of more than one year}

9. The ceiling on nonconcessional external debt applies to external debt contracted or guaranteed by the central government, the BM, the Road Fund, the water authorities (FIPAG), and the electricity company (EDM). It also applies to debt contracted by these three state-owned enterprises from domestic banks or from other state-owned enterprises that is contractually interrelated to external nonconcessional loans.

10. The ceiling applies to external debt with original maturity of one year or more and with a grant element below 35 percent. The grant element is calculated using currency-specific discount rates based on the Organization for Economic Cooperation and Development (OECD) commercial interest reference rates in accordance with the annual Budget Law. The term 'debt' will have the meaning set forth in Point 9 of the Guidelines on Performance Criteria with Respect to External Debt in Fund Arrangements adopted on August 3, 1979, as amended August 31, 2009, effective December 1, 2009. The concept of external debt is defined on the basis of the residency of the creditor. The ceiling also applies to commitments contracted or guaranteed for which value has not been received. This assessment criterion is defined cumulatively from the beginning of the program and will be assessed on a continuous basis.

\section{Stock of short-term external public debt outstanding}

11. The central government will not contract or guarantee external debt with original maturity of less than one year. This assessment criterion applies not only to debt as defined in Point 9 of the Guidelines on Performance Criteria with Respect to External Debt in Fund Arrangements adopted on August 3, 1979, as amended August 31, 2009, effective December 1, 2009, but also to commitments contracted or guaranteed for which value has not been received. Excluded from this assessment criterion are short-term, import-related trade credits. This assessment criterion will be assessed on a continuous basis. 


\section{External payments arrears}

12. The government undertakes not to incur payments arrears on external debt owed or guaranteed by the central government, with the exception of external payments arrears arising from government debt that is being renegotiated with creditors. This assessment criterion will be assessed on a continuous basis.

\section{Foreign program assistance}

13. Foreign program assistance is defined as grants and loans received by the Ministry of Finance through BM accounts excluding those related to projects (Table 1).

\section{Actual external debt service payments}

14. Actual external debt service payments are defined as cash payments on external debt service obligations of the government and central bank, including obligations to Paris Club and other bilateral creditors rescheduled under enhanced HIPC Initiative completion point terms, multilateral creditors, and private creditors, but excluding obligations to the IMF (Table 1).

\section{ADJUSTERS}

\section{Net international reserves}

15. The quantitative targets (floors) for net international reserves (NIR) will be adjusted:

- downward by the shortfall in external program aid less debt service payments (up to US\$100 million), compared to the program baseline (Table 1);

- downward/upward for any revision made to the end-year figures corresponding to the previous year; and

- downward to accommodate higher external outlays because of natural disasters, up to US\$20 million.

\section{Net credit to central government}

16. The quantitative targets (ceilings) for net credit to the central government (NCG) will be adjusted:

- upward by the shortfall in the MT value of external program aid receipts less debt service payments (up to the MT equivalent of US $\$ 100$ million at exchange rates prevailing at the respective test dates), compared to the program baseline (Table 1);

- downward by privatization proceeds in excess of those envisaged in the program, unless these proceeds are deposited in the government's savings accounts abroad;

- downward (upward) for any increase (decrease) in domestic financing from the nonfinancial private sector; and

- upward to accommodate the higher locally-financed outlays because of natural disasters, up to the MT equivalent of US\$20 million at exchange rates prevailing at the respective test dates. 


\section{Reserve money}

17. The quantitative target (ceiling) for reserve money will be adjusted upward by the excess of the stock of currency in circulation above the level envisaged in the program. For programming purposes, both the stocks of reserve money and currency in circulation are defined in terms of the average of the daily end-of-day stocks in the month of the test date. The target will be adjusted up to MT 500 million for end-March, end-June, and end-September and up to MT 750 million for end-December (Table 1).

18. The ceiling on reserve money for every test date will be adjusted downward/upward to reflect decreases/increases in the legal reserve requirement on the liabilities in commercial banks. The adjuster will be calculated as the change in the reserve requirement coefficient multiplied by the amount of commercial banks' liabilities subject to reserve requirement, considered at the end of the period of constitution of the required reserves prior to the change in regulation.

\section{DATA AND OTher REPORTING}

19. The government will provide Fund staff with:

- monthly and quarterly data needed to monitor program implementation in relation to the program's quantitative targets and broader economic developments;

- $\quad$ weekly updates of the daily data set out in Table 1;

- weekly data set out in Table 4 of the TMU dated May 26, 2005;

- monthly updates of the foreign exchange cash flow of the BM;

- monthly data on government revenues (in detail according to the fiscal table) with a lag not exceeding one month;

- monthly information on the balance of government savings accounts abroad;

- monthly data on domestic arrears;

- monthly budget execution reports (that will also be published) with a time lag not exceeding 45 days;

- monthly monetary survey data with a time lag not exceeding 30 days; and

- monthly data on gross international reserves, with the composition by original currencies and converted to US dollars at the actual exchange rates.

20. The monetary survey made available by the BM will clearly identify donor-financed project deposits (with a breakdown between foreign and domestic currency) included in net credit to the government in both the central bank's and commercial banks' balance sheets.

21. The government will provide Fund staff with documentation concerning external loan agreements once these have been signed and become effective. 
TMU Table 1. Mozambique: Net Foreign Assistance, 2010-11

\begin{tabular}{|c|c|c|c|c|c|c|c|c|c|c|c|c|c|c|c|c|}
\hline & \multicolumn{10}{|c|}{2010} & \multicolumn{6}{|c|}{2011} \\
\hline & \multicolumn{2}{|c|}{ Q1 } & \multicolumn{2}{|l|}{ Q2 } & \multicolumn{2}{|l|}{ Q3 } & \multicolumn{2}{|l|}{ Q4 } & \multicolumn{2}{|c|}{ Year } & \multirow{2}{*}{$\frac{\text { Q1 }}{\text { Prog. }}$} & \multicolumn{2}{|c|}{ Q2 } & \multirow{2}{*}{$\begin{array}{r}\text { Q3 } \\
\text { Proj. } \\
\end{array}$} & \multirow{2}{*}{$\begin{array}{r}\text { Q4 } \\
\text { Proj. }\end{array}$} & \multirow{2}{*}{$\begin{array}{l}\text { Year } \\
\text { Proj. }\end{array}$} \\
\hline & Prog. & Act. & Prog. & Act. & Prog. & Act. & Prog. & Act. & Prog. & Act. & & Prog. & Proj. & & & \\
\hline Net foreign program assistance (US\$ mn) & 163 & 58 & 181 & 152 & 27 & 101 & 115 & 71 & 396 & 382 & 129 & 106 & 126 & 104 & 104 & 394 \\
\hline Gross foreign program assistance & 182 & 74 & 203 & 168 & 42 & 117 & 132 & 88 & 467 & 447 & 144 & 121 & 140 & 119 & 118 & 453 \\
\hline Program grants & 151 & 74 & 176 & 138 & 42 & 117 & 50 & 5 & 355 & 335 & 144 & 89 & 140 & 119 & 3 & 338 \\
\hline Program loans & 30 & 0 & 27 & 30 & 0 & 0 & 82 & 83 & 112 & 112 & 0 & 32 & 0 & 0 & 116 & 116 \\
\hline External debt service & 18 & 16 & 22 & 16 & 15 & 16 & 18 & 16 & 71 & 65 & 15 & 15 & 15 & 15 & 15 & 60 \\
\hline Net foreign program assistance (MT mn) & 4,843 & 1,661 & 5,378 & 5,184 & 829 & 3,633 & 3,975 & 2,428 & 12,935 & 12,905 & 4,732 & 3,838 & 3,802 & 3,170 & 3,224 & 12,065 \\
\hline Gross foreign program assistance & 5,220 & 2,076 & 6,021 & 5,673 & 1,278 & 4,097 & 4,738 & 2,905 & 15,775 & 14,751 & 5,253 & 4,375 & 4,337 & 3,693 & 3,765 & 14,187 \\
\hline Program grants & 4,344 & 2,076 & 5,229 & 4,659 & 1,278 & 4,097 & 1,798 & 162 & 11,821 & 10,994 & 5,253 & 3,229 & 4,337 & 3,693 & 93 & 10,516 \\
\hline Program loans & 876 & 0 & 792 & 1,014 & 0 & 0 & 2,940 & 2,743 & 3,954 & 3,757 & 0 & 1,147 & 0 & 0 & 3,672 & 3,672 \\
\hline External debt service & 376 & 416 & 643 & 489 & 449 & 464 & 763 & 477 & 2,840 & 1,846 & 521 & 537 & 535 & 523 & 541 & 2,123 \\
\hline Stock of outstanding currency (MTm) & 13,278 & 14,515 & 16,445 & 16,721 & 16,436 & 18,364 & 19,134 & 20,447 & 19,134 & 20,447 & 16,098 & 17,556 & 19,164 & 22,512 & 24,147 & 24,147 \\
\hline Stock of outstanding currency (MTm), Prog. Def & 13,278 & 14,317 & 15,324 & 16,080 & 16,052 & 17,866 & 18,616 & 20,403 & 18,616 & 20,403 & 15,878 & 16,883 & 18,430 & 21,901 & 24,096 & 24,096 \\
\hline
\end{tabular}




\section{APPENDIX II: EXChANGE RATE ASSESSMENT ${ }^{1}$}

1. In 2010, the metical depreciated against the currencies of major trading partners. It depreciated by 24 percent against the dollar, 10 percent against the euro, and 26 percent against the rand. In 2010, the United States accounted for about 4 percent of total trade, the Euro area for 32 percent, and South Africa for 41 percent. Relative to its peak in November of 2008 , the metical depreciated by 33 percent in real effective terms, mainly driven by a 40 percent depreciation in nominal effective terms.

\section{A qualitative exchange rate assessment suggests that the value of the real} effective exchange rate is broadly in line with medium-term fundamentals. Macroeconomic projections conditional on a constant real effective exchange rate indicate sustainable balance of payments dynamics over the medium term. The current account deficit is projected to stabilize at 101/2 percent of GDP by 2015, while gross international reserves should approach 5 months of imports. The current account deficit is primarily financed by relatively stable long-term private capital inflows related to mega-project investments and public capital inflows on concessional terms.

3. A quantitative exchange rate assessment suggests a small undervaluation of the REER, with a mid-point estimate of the three standard methodologies of 1.5 percent. ${ }^{2,3}$ Under the macroeconomic balance approach, the estimated current account norm of -9 percent of GDP is below the projected underlying current account of -10.5 percent of GDP, implying a slight overvaluation of 0.5 percent. Under the external sustainability approach, the most recently observed NFA position of -80 percent of GDP implies a current account norm of -9.5 percent of GDP, which translates into an undervaluation of 1 percent. The equilibrium real exchange rate approach suggests an undervaluation of about 4 percent.

\section{While empirical findings suggest that the previous large overvaluation has been} corrected, external competitiveness remains a challenge in Mozambique (Figure 1). It has shown little progress under various survey-based indicators, such as the Doing Business and Global Competitiveness Index.

5. These results suggest that restoring external price competitiveness is no longer a pressing issue. Further improving external competitiveness rests on continuing efforts to enhance institutions, infrastructure, the provision of health and education services, and on determined steps to improve the business climate.

\footnotetext{
${ }^{1}$ Prepared by Karina Garcia (SPR).

${ }^{2}$ For a description of the empirical methodology, see Vitek, F. (2009): Exchange rate assessment tools for advanced, emerging, and developing economies. IMF internal document.

${ }^{3}$ Results under the three CGER methodologies should be interpreted carefully as data shortcomings (short sample periods and lack of data the needs to be proxied for) may increase the uncertainty surrounding econometric estimates.
} 


\section{Figure 1. Mozambique: Real Exchange Rate and Competitiveness}

Through end-2010, the metical continued depreciating against currencies of major trading partners...

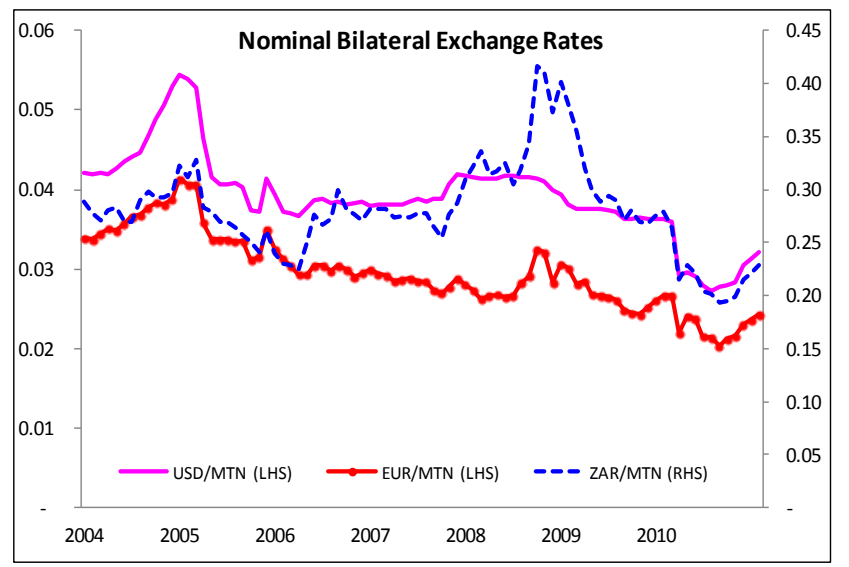

Empirical evidence of exchange rate misalignment suggests a modest undervaluation...

\begin{tabular}{|c|c|c|c|}
\hline \multicolumn{4}{|c|}{ Summary of Quantitative Exchange Rate Assesment Results ${ }^{1}$} \\
\hline & \multicolumn{2}{|c|}{ CA/GDP } & REER \\
\hline & Norm & \multicolumn{2}{|c|}{ Underlying $^{4}$} \\
\hline $\mathrm{MB}^{2}$ & -9.0 & -9.2 & 0.5 \\
\hline ERER & $\ldots$ & $\ldots$ & -3.7 \\
\hline$E S^{3}$ & -9.5 & -9.2 & -1.2 \\
\hline \multicolumn{4}{|c|}{${ }^{1}$ Based on March 2011 WEO. } \\
\hline \multicolumn{4}{|c|}{$\begin{array}{l}2 \text { Based on calibrated semi elasticity of }-0.28 \text {. See Tokarick, 2009, A Method for } \\
\text { Calculating Export Supply and Import Demand Elasticities. }\end{array}$} \\
\hline \multicolumn{4}{|c|}{${ }^{3}$ Based on projected medium term nominal output growth of $13 \mathrm{pct}$. } \\
\hline \multicolumn{4}{|c|}{${ }^{4}$ Projection conditional on a constant real effective exchange rate. } \\
\hline
\end{tabular}

...external competitiveness remains a challenge as Mozambique figures at the bottom of the competitive ladder relative to its peers.

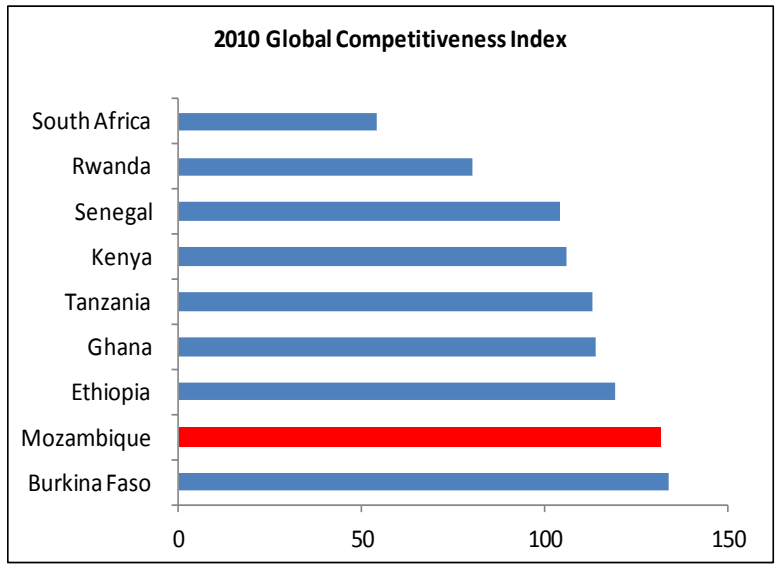

...contributing to the depreciation in real and nominal effective terms.

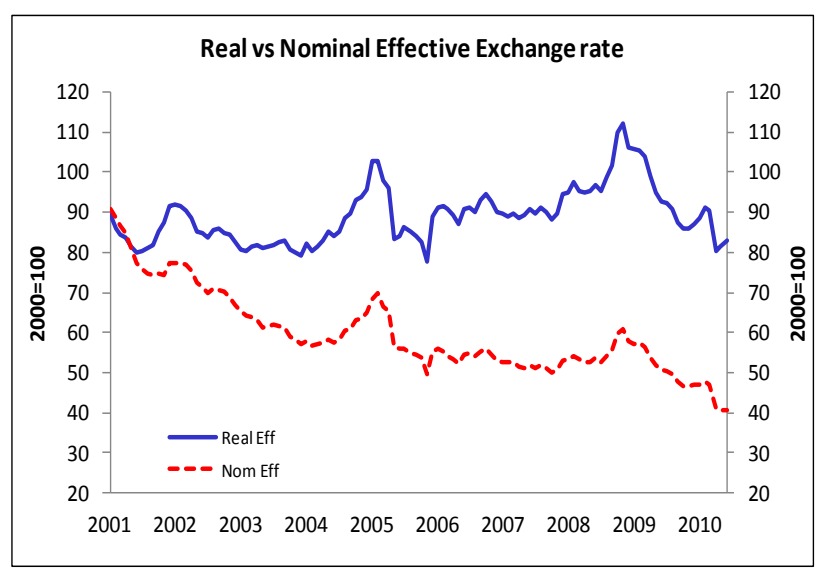

...indicating a substantial correction of previous overvaluations; however...

\begin{tabular}{|lccc|}
\hline & Current Assessment & Fall 2009 $^{1}$ & 2009 Art IV $^{-1}$ \\
\hline MB & 0.5 & 25.0 & 25.6 \\
ES & -3.7 & 10.6 & 40.8 \\
ERER & -1.2 & -2.5 & 38.4 \\
Misalignment mid-point range & -1.5 & 11.0 & 34.9 \\
\hline Memo items & & & \\
Underlying CA & -9.2 & -9.9 & -7.8 \\
Equilibrium CA (MB) & -9.0 & -6.2 & -3.8 \\
Equilibrium CA (ES) & -9.5 & -7.9 & -1.9 \\
\hline A Ap)
\end{tabular}

A preliminary update of exchange rate assesment was done using data as of October 2009.

Changes in the assesment reflect a large nominal and real depreciation through 2010 as well as refinements in the methodology.

Improving external competitiveness requires enhancing institutions as well as the business climate.

\begin{tabular}{|lccc|}
\hline & \multicolumn{3}{c|}{ Doing Business Rank } \\
\cline { 2 - 4 } Topic Rankings & $\mathbf{2 0 1 1}$ & $\mathbf{2 0 1 0}$ & Change \\
\hline Overall ranking & $\mathbf{1 2 6}$ & $\mathbf{1 3 0}$ & $\mathbf{4}$ \\
\hline Starting a Business & 65 & 96 & 31 \\
Dealing with Construction Permits & 155 & 159 & 4 \\
Registering Property & 144 & 153 & 9 \\
Getting Credit & 128 & 125 & -3 \\
Protecting Investors & 44 & 41 & -3 \\
Paying Taxes & 101 & 98 & -3 \\
Trading Across Borders & 133 & 136 & 3 \\
Enforcing Contracts & 132 & 129 & -3 \\
Closing a Business & 129 & 135 & 6 \\
\hline
\end{tabular}

Sources: IMF staff projections and estimates; World Bank, Doing Business Report; World Economic Forum. 


\section{APPENDIX III: THE MACROECONOMICS OF INCLUSIVE GROWTH ${ }^{1}$}

Notwithstanding Mozambique's high real GDP growth over the last two decades, economic growth has not been as pro-poor as in other countries with comparable high and sustainable growth episodes. It has also become less pro-poor over time. Making economic growth more inclusive in Mozambique will require policies and reforms to diversify the country's productive and export base and raise production and productivity in labor-intensive industries, especially agriculture. These policies should be supported by improvements in the macroeconomic management of natural resource wealth to help close the infrastructure and skills gaps and expand social protection systems. Broader, well-targeted, and fiscally sustainable social protection systems would also be crucial to mitigate the economic impact of rising income inequality, thereby ensuring social cohesion and avoiding growth collapses.

\section{What IS INCLUSIVE GROWTH?}

\section{Traditionally, the elements widely considered as being conducive to economic} growth are: ${ }^{2}$ (i) macroeconomic stability, including moderate fiscal and current account deficits, a low debt-to-GDP ratio, low and stable inflation, and adequate international reserve levels; (ii) high investment and savings rates in physical and human capital; (iii) openness to trade; (iv) enforcement of property rights and the rule of law; and (v) good governance.

2. Inclusive growth goes beyond this concept. It is defined as growth that is sustained over decades, is broad-based across economic sectors, creates productive employment opportunities for the majority of the country's population, and reduces poverty noticeably. ${ }^{3}$

\section{As a result, in order for growth to be considered as inclusive, several additional} elements are needed: (i) high growth rates that are sustained for several years, through a combination of more frequent and sustained growth accelerations and, importantly, by avoiding growth collapses; (ii) the structural transformation of the economy, including the diversification of output and exports, the transition from an agriculture-based to a manufacturing- and services-based economy, and the migration within manufacturing and services from low value-added to high value-added activities; (iii) productive employment, defined as employment growth accompanied by productivity growth; (iv) significant reduction in absolute poverty; ${ }^{4}$ (iv) a propitious business environment or investment climate;

\footnotetext{
${ }^{1}$ Prepared by Victor Lledó and Rodrigo Garcia-Verdu. This note draws from a presentation prepared for the high-level conference on Challenges for Economic Growth and Job Creation in Maputo February 9-11, 2011.

${ }^{2}$ See Commission on Growth and Development (2008).

${ }^{3}$ See the definitions by the Commission on Growth and Development (2008) and Ianchovichina and Lundstrom (2009).

${ }^{4}$ The inclusive growth definition is more in line with the absolute definition of pro-poor growth. Under the absolute definition, growth is considered to be pro-poor as long as poor people benefit in absolute terms, as reflected in some agreed measure of poverty (Ravallion and Chen, 2003). It is less in line with the relative definition, whereby growth is pro-poor if, and only if, the incomes of poor people grow faster than those of the population as a whole, i.e., inequality declines.
} 
(v) efficient natural resource management; and (vi) equality of opportunity through investments in education, health, and infrastructure.

\section{How InCluSive has Growth been In MozambiQue?}

4. Economic growth in Mozambique, despite its high rates, has not been as propoor as in other successful countries, and has become less pro-poor over time (Figure 1).

- Mozambique's economic growth averaged 8 percent over the period 1995-2009, the second highest among non fuel-exporting countries in the region. Real GDP per capita almost doubled after 1992. This performance has been anchored on sound macroeconomic management and structural reforms.

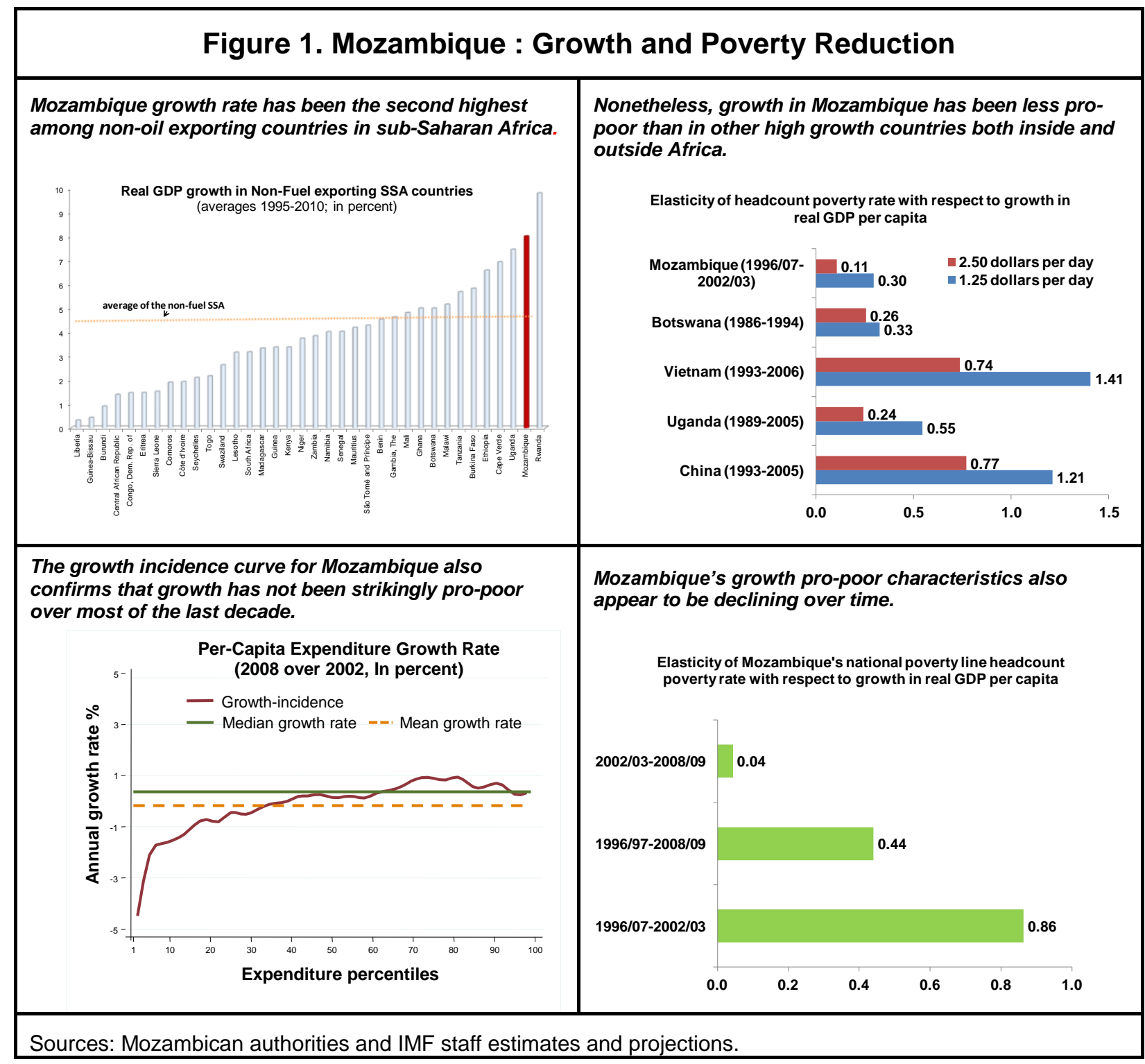


- $\quad$ Poverty reduction has initially been significant. The poverty headcount fell from 69 percent in 1997 to 54 percent in 2003 as consumption per capita grew by a cumulative 50 percent over the same period. Reduction in rural poverty was even more pronounced, declining from 71 to 55 percent during the same period.

- However significant, poverty reduction did not reach the magnitude observed in other high-growth countries. Cross-country estimates of the elasticity of the headline poverty rate with respect to the growth of real GDP per capita suggest an average elasticity close to 0.20 , which is only a fraction of that observed in countries like China, Vietnam, and even Uganda.

- Similarly, preliminary evidence from household survey data shows that over the 2002/03 to 2008/09 period, growth has not followed an inclusive or pro-poor pattern. In particular, the growth incidence curve shows that households in the bottom three deciles of the distribution of per-capita expenditure experienced absolute declines, while the other seven deciles experienced positive growth. This sharply contrasts the experience of other countries, such as China and especially Brazil, over the last three to four decades.

- Not only has growth in Mozambique been less pro-poor than in other successful countries, but such pro-poor characteristics appear to be declining over time. With growth remaining relatively high, this reflects stagnant poverty numbers. The latest National Poverty Report (MPD-DNEAP, 2010) suggests that the poverty rate in 2008 has remained high and stagnant, at about 54 percent, with rural poverty increasing to 57 percent.

5. Moreover, economic growth in Mozambique still needs to be sustained for several more years, and be broader and more diverse, to become more inclusive.

- In terms of the length of Mozambique's growth take-off which started around 1992, it still needs to be sustained for several more decades to match successful growth cases. Indeed, all the countries considered by the Growth Commission as successful cases of inclusive development have sustained high growth rates for at least three decades.

- Growth has not been accompanied by economic diversification either. Measured by the Herfindahl-Hirschman index, Mozambique's export base has become increasingly concentrated - a reflection of the emergence of megaprojects. This contrast with the experience of countries like Uganda, which was able to successfully diversify its exports notwithstanding its low level of initial GDP per capita, its protracted civil conflict, and the fact that it is landlocked. 
Figure 2. Mozambique: Growth and Structural Transformation

Despite Mozambique's impressive performance over the past two decades, growth still needs to be sustained for several more years...

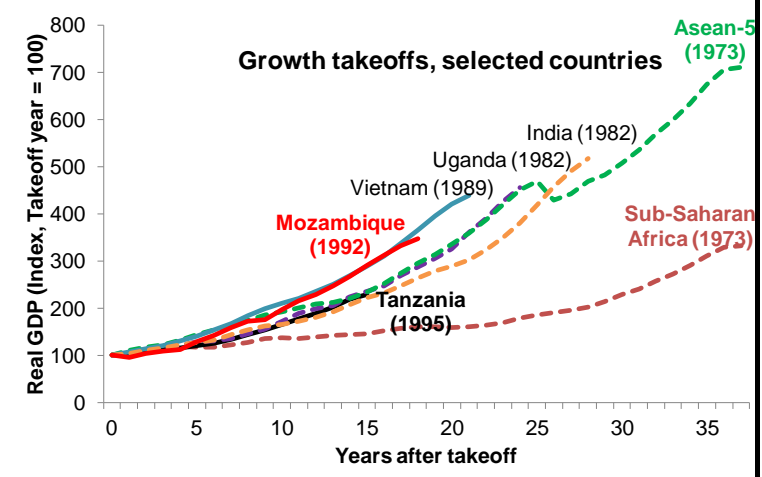

....and be accompanied by greater economic diversification.

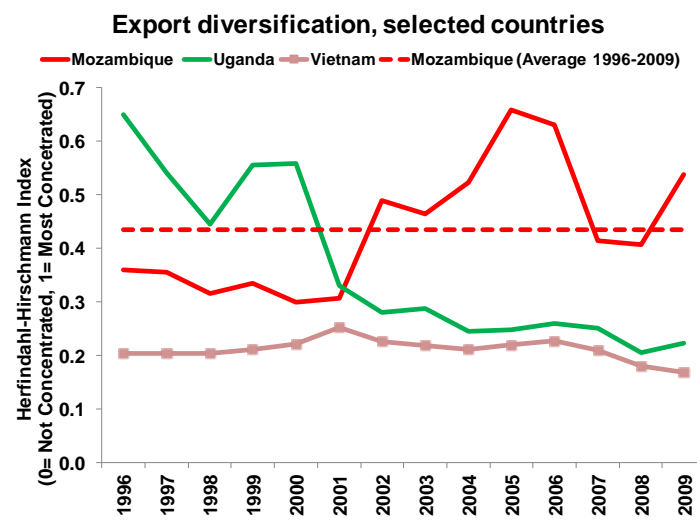

Sources: Mozambican authorities and IMF staff estimates and projections.

\section{InClusive Growth Strategies, Policies, And Reforms}

6. Mozambique needs to make greater progress towards more inclusive growth. In particular, it needs to: (i) accelerate growth while avoiding growth collapses; (ii) diversify its economy away from capital-intensive, low-value added products; (iii) increase production and productivity in labor-intensive sectors, particularly in agriculture (Box 1); and (iv) promote equality of opportunities, by leveling the playing field among households and firms. All these elements have proved fundamental elsewhere in order to sustain rapid poverty reduction.

7. In all these endeavors, Mozambique should preserve macroeconomic stabilityone of Mozambique's key assets. Much of the post-1995 improvement in economic performance in Mozambique is attributable to avoiding growth collapses. To a large extent, this was the direct result of the implementation of policies and reforms to keep inflation low and to achieve, and then preserve, debt sustainability.

\section{Country-specific growth opportunities and challenges will also play an} important role in inclusive growth strategies. In the case of Mozambique, geography and geology matter. Mozambique is a coastal country and a neighbor of a large regional market: South Africa. Promoting exports to better tap into regional and international markets has proved a very successful strategy in other coastal developing countries, particularly those with very small domestic markets. 


\section{Box 1 : Increasing Agricultural Production and Productivity in Mozambique ${ }^{1}$}

Increasing agricultural production and productivity needs to assume central stage in Mozambique's inclusive growth strategy. It will be critical to foster pro-poor growth, as it could create more productive and better-paid job opportunities for the 80 percent of the Mozambican population living in rural areas, most of it locked in subsistence agriculture. Productive gains in agriculture would also be a necessary condition for the structural transformation of the economy, as it endows the surplus labor released from agricultural activities with additional skills to facilitate the transition towards an industrialized economy and eventually to a services-based economy (McCalla 2011). Mozambique is still in an early phase of this transition. In fact, the share of the population employed in agriculture experienced little change between 2002/03 and 2008/09, which suggests stagnant agricultural productivity (Fox 2011).

Mozambique has great agricultural potential. Deninger (2011) estimates that Mozambique is among the ten countries with the greatest unexploited farming potential. Mozambique, along with other countries located in the African Guinea Savannah, also has the right agro-climatic conditions for obtaining very high yields in the production of important food staples, including cassava, corn, and rice, as well as cash crops such as sugar and cotton (Morris 2011). A number of countries with similar agro-climatic and factor endowments, such as Brazil and Thailand, have succeeded in getting agriculture going.

After having made agriculture more productive, Mozambique can further accelerate the transition away from agriculture and into manufacturing and services, by: (i) improving educational levels; (ii) enhancing the business environment, particularly in labor-intensive sectors; (iii) supporting start-ups and improve productivity of the selfemployed; and (iv) increasing access to markets through better infrastructure (Fox 2011).

\section{The experiences of Brazil and China show that agricultural productivity can be} improved through coordinated policies: first, through a combination of price liberalization (e.g., dismantling of control prices and boards and elimination of export taxes) and agricultural policies that move away from direct subsidies for certain products (e.g., wheat flour in Mozambique) and towards the provision of public goods such as basic research, infrastructure, and agricultural extension services (de França 2011, Li 2011); and second, through subsidized credit policies in the form of credit guarantees (Morris 2011). In the case of Mozambique, it is also particularly important to have a clear definition of property rights, akin to China's reform in the late 1970s which set the stage for subsequent increases in agricultural productivity.

${ }^{1}$ Based on presentations on agriculture at high-level conference on Challenges for Economic Growth and Job Creation in Maputo, February 9-11, 2011. 
9. However, to foster inclusive growth, export promotion will need to be tackled through export diversification strategies that boost productivity and, hence, competiveness in labor-intensive sectors. Countries that have successfully implemented this strategy have done so by: (i) stepping up public investment to remove infrastructure and skills gaps; (ii) accelerating regulatory reforms to improve the business environment; (iii) improving access to finance to small and medium-sized enterprises; and (iv) optimizing market access and regional integration. Leadership and intergovernmental coordination has also proved to be a critical element. In most successful cases, the President or the Prime Minister led the implementation effort, helped by a small office directly accountable to him/her, with coordination powers.

\section{Export diversification needs to be supported by a strategy that improves the} management of natural resources. Mozambique is a resource-rich country, with an abundance of minerals, gas, coal, and now even oil. To avoid the resource curse, Mozambique will need to sustain the reform momentum to ensure that natural resources are managed well. It would be particularly important to avoid the "resource curse" by setting the right institutions at an early stage. Elements of a successful strategy for mineral revenue management include: (i) getting a fair price for mineral resources through appropriate contracts, concessions, and taxation schemes; (ii) improving revenue transparency, among other things by acceding to the EITI on a timely basis; (iii) establishing special fiscal institutions to smooth revenues across the economic cycle, thus avoiding the typical boombust cycles; (iv) continuing to improve public financial management systems to ensure that spending is properly and efficiently allocated; and (v) an appropriate monetary and exchange rate policy that avoids the emergence of Dutch disease and suffocating traditional export sectors.

11. Broader, well-targeted, and fiscally sustainable social protection systems would also be crucial to prevent income inequality from rising, thereby ensuring social cohesion and avoiding growth collapses. ${ }^{5}$ As income inequality rises, so does the risk of social conflict — as the recent events in the Middle East provide a topical reminder of. One way of accelerating poverty reduction and mitigating the effects of increasing inequality is through the introduction of a targeted social safety net, such as conditional cash transfer schemes, such as Bolsa Familia in Brazil, or public work programs, such as those in India and Ethiopia. These programs have a relatively low fiscal cost and have been shown to positively affect labor productivity.

\footnotetext{
${ }^{5}$ Drawing on previous work showing that countries with more equal income distributions tend to have significantly longer growth spells and less frequent growth collapses, Berg and Ostry (2011) suggest welltargeted subsidies and social protection measures as successful policies that could be capable of improving income distribution without undermining incentives.
} 


\section{References}

Commission on Growth and Development (2008), The Growth Report. Strategies for Sustained Growth and Inclusive Development, Washington D.C.: The World Bank.

de França, José Geraldo Eugênio (2011), "Brazil’s secret: Lifting 30 million out of poverty EMBRAPA," Presentation at the Government of Mozambique Seminar on Broad-Based Development through Economic Transformation, and Job Creation 9-11 February 2011, Maputo, Mozambique.

Deininger, Klaus (2011), "Land Policy and Rural Development Reforms for Improving land use in Mozambique: Valuation, taxation, and land use planning," Presentation at the Government of Mozambique Seminar on Broad-Based Development through Economic Transformation, and Job Creation 9-11 February 2011, Maputo, Mozambique.

Fox, Louise (2011), "Employment and Job Creation in Mozambique," Presentation at the Government of Mozambique Seminar on Broad-Based Development through Economic Transformation, and Job Creation 9-11 February 2011, Maputo, Mozambique.

Ianchovichina, Elena, and Susanna Lundstrom (2009), "Inclusive Growth Analytics. Framework and Application," Policy Research Working Paper 4851, Washington D.C.: The World Bank.

Li, Guo (2011), "Rural Development Reforms in China," Presentation at the Government of Mozambique Seminar on Broad-Based Development through Economic Transformation, and Job Creation 9-11 February 2011, Maputo, Mozambique.

McCalla, Alex F. (2011), "The Role of Agriculture in Economic Development," Presentation at the Government of Mozambique Seminar on Broad-Based Development through Economic Transformation, and Job Creation 9-11 February 2011, Maputo, Mozambique.

Minister of Planning and Development, National Directorate of Studies and Policy Analysis (MPDDNP), October 2010. "Poverty and Well-Being in Mozambique: Third National Poverty Assessment, Ministry of. Pobreza e Bem-estar em Moçambique: Terceira Avaliação Nacional da Pobreza, Ministério da Planificação e Desenvolvimento, Direcção Nacional de Estudos e Análise de Políticas.

Morris, Michael (2011), “Awakening Africa’s Sleeping Giant - Lessons for Raising Agricultural Competitiveness ," Presentation at the Government of Mozambique Seminar on Broad-Based Development through Economic Transformation, and Job Creation 9-11 February 2011, Maputo, Mozambique. 


\section{APPENDIX IV.STRESS-TeSTING OF THE BANKING SECTOR ${ }^{1}$}

Stress-testing undertaken during Mozambique's 2009 FSAP update based on September 2008 data was replicated with September 2010 data. The results do not vary significantly (see Summary Table): The level of capitalization appears sufficient to withstand plausible macroeconomic shocks; the concentration of loans on single borrowers remains a major vulnerability; the direct exchange rate risk on banks' capitalization is likely to be limited; and liquidity conditions have somewhat tightened over the recent period.

\section{The Mozambican banking system has recently expanded and remains highly} concentrated. The number of banks operating in the market has increased from 14 in 2008 to 16 at end-2010. The size of the sector's assets has been growing rapidly as a result of the expansion of the three largest banks reflecting strong credit growth (from 18.7 percent of GDP at end-2008 to 28.2 percent of GDP at end-2010). The concentration of the sector has remained very high: The three (four) largest banks, all foreign-owned, account for about 80 (90) percent of the system's assets.

\section{The sensitivity tests broadly used the same methodology as for the 2009 FSAP}

update. The stress tests assume uniform shocks. In particular, new nonperforming loans (NPLs) stemming from the shocks are presumed fully provisioned and reducing the regulatory capital in due proportion. Capital adequacy ratios (CARs) before shocks are corrected on the basis of the following lump provisioning rate on existing loans: 1 percent on performing loans, 33 percent on nonperforming loans, and 100 percent on losses. ${ }^{2}$ The macro financial shock of a drop in exports, foreign aid, and FDI used the same elasticity coefficient of -3 to estimate the effect of a GDP growth decline on NPLs. The whole exercise excludes an outlier undercapitalized medium-sized bank on which the authorities should take corrective action.

3. The banking system appears similarly resilient to a large shock on external sources of growth. A large shock combining a 25 percent decline in real export volumes, foreign aid and private FDI, causing real GDP growth to decline by 5.3 percentage points, would lead to moderate decreases in CARs. The results suggest that the top three banks would better withstand the suggested external shocks than medium-small banks.

4. Concentration on the largest exposures remains a major risk to banks' solvency. Should each bank's largest borrower default, the largest banks' and the system-wide capital adequacy ratio could be jeopardized. This is because primary borrowers' risks account for about 65 percent of banks' regulatory capital on average. Sector or industry-specific shocks were not simulated in the absence of detailed information on large exposures.

\footnotetext{
${ }^{1}$ Prepared by Patrick Gitton.

${ }^{2}$ Absent recent data, the results and analysis do not reflect off-balance sheet exposures.
} 


\section{Shocks on balance-sheet foreign exchange open positions still suggest a limited} impact on banks' capitalization. Most banks reported a moderate and positive net open foreign exchange position, thereby benefiting from potential depreciation of the domestic currency. The regulatory requirement to ex ante provision 50 percent of foreign exchange loans to non-exporters circumscribes FX-related credit risks on unhedged borrowers. The risk of a potential currency mismatch should nevertheless be monitored as, system-wide, foreign currency deposits largely exceed foreign currency loans.

\section{Liquidity shocks would have a more pronounced effect on the largest banks.}

Liquidity ratios have generally decreased over the past two years, partly reflecting strong credit growth. The largest banks would be more vulnerable to a simulated impact of a 30 percent drop in all deposits and 20 percent drop in demand deposits. Generally, though, as the interbank market remains shallow (domestic interbank gross credit is very limited), direct interbank contagion is unlikely. 
SUMMARY OF STRESS TEST RESULTS, as of September 2010

\begin{tabular}{|c|c|c|c|c|}
\hline \multirow[b]{3}{*}{ Banking System Structure (before shock) } & \multirow{2}{*}{$\begin{array}{c}2009 \text { FSAP Update } \\
\text { Banking System } \\
\end{array}$} & \multicolumn{3}{|c|}{2010} \\
\hline & & Banking System & Top 3 banks & Medium-Small banks \\
\hline & & & & \\
\hline Number of Analyzed Banks 1/ & 12 & 12 & 3 & 9 \\
\hline Assets (mln MT) & 90,722 & 196,505 & 161,071 & 35,434 \\
\hline Regulatory Capital (mln MT) & 7,394 & 13,595 & 10,670 & 2,924 \\
\hline CAR & 14.4 & 15.4 & 14.9 & 17.2 \\
\hline NPLs & 2.1 & 1.8 & 0.9 & 6.4 \\
\hline Corrected CAR for Underprovisioning & 13.8 & 14.8 & 14.4 & 16.9 \\
\hline \multicolumn{5}{|c|}{ MACRO CREDIT RISK } \\
\hline \multicolumn{5}{|c|}{ Stress-Scenario 1: 25 percent Aid, Export and FDI shock } \\
\hline CAR & 13.5 & 14.3 & 14.1 & 15.2 \\
\hline Change in CAR in Percentage Points & -0.3 & -0.5 & -0.3 & -1.6 \\
\hline Number of Banks with CAR $<8$ & 3 & 0 & 0 & 0 \\
\hline Number of Insolvent Banks with CAR $<0$ & 1 & 0 & 0 & 0 \\
\hline NPL (\% of loans) & 2.5 & 2.3 & 1.2 & 8.4 \\
\hline \multicolumn{5}{|c|}{ Stress-Scenario 2: 25 percent export receipts shock } \\
\hline CAR & 13.1 & 14.5 & 14.2 & 15.9 \\
\hline Change in CAR in Percentage Points & -0.7 & -0.3 & -0.2 & -1.0 \\
\hline Number of Banks with CAR $<8$ & 2 & 0 & 0 & 0 \\
\hline Number of Insolvent Banks with CAR $<0$ & 0 & 0 & 0 & 0 \\
\hline NPL ( $\%$ of loans) & 2.5 & 2.0 & 1.1 & 7.5 \\
\hline \multicolumn{5}{|l|}{ CONCENTRATION RISK } \\
\hline \multicolumn{5}{|c|}{ Stress-Scenario: default of larger borrower per bank } \\
\hline CAR & 7.8 & 5.3 & 4.2 & 9.8 \\
\hline Change in CAR in Percentage Points & 6.0 & -9.5 & -10.2 & -7.1 \\
\hline Number of Banks with CAR $<8$ & 3 & 6 & 3 & 3 \\
\hline Number of Insolvent Banks with CAR $<0$ & 1 & 1 & 1 & 0 \\
\hline NPL ( $\%$ of Loans) & 9.1 & 11.3 & 10.5 & 16.1 \\
\hline \multicolumn{5}{|c|}{ DIRECT EXCHANGE RATE RISK } \\
\hline \multicolumn{5}{|c|}{ Stress-Scenario 1: 25 Depreciation against the USD } \\
\hline CAR & 14.3 & 15.0 & 14.7 & 16.6 \\
\hline Change in CAR in Percentage Points & 1.6 & 0.3 & 0.3 & 0.2 \\
\hline Capital Loss/ Gain (in \% of Capital) & 6.0 & 1.9 & 2.1 & 1.1 \\
\hline \multicolumn{5}{|c|}{ Stress-Scenario 2: 50 Depreciation against the USD } \\
\hline CAR & 15.1 & 15.3 & 15.0 & 16.7 \\
\hline Change in CAR in Percentage Points & 0.8 & 0.6 & 0.6 & 0.4 \\
\hline Capital Loss/ Gain (in \% of Capital) & 12.0 & 3.8 & 4.3 & 2.2 \\
\hline \multicolumn{5}{|l|}{ LIQUIDITY RISK } \\
\hline Liquid Assets (mln MT) 1/ & 27,971 & 58,100 & 45,900 & 12,343 \\
\hline Liquid Liabilities (mln MT) 1/ & 35,304 & 119,093 & 72,604 & 15,163 \\
\hline Liquidity Ratio $2 /$ & $79 \%$ & $66 \%$ & $63 \%$ & $81 \%$ \\
\hline \multicolumn{5}{|c|}{ Stress Scenario 1: General deposit decrease of 20 percent } \\
\hline Change in Liquidity Ratio & $-12 \%$ & $-12 \%$ & $-14 \%$ & $-6 \%$ \\
\hline \multicolumn{5}{|c|}{ Stress Scenario 2: Demand deposit decrease of 30 percent } \\
\hline Change in Liquidity Ratio & $-9 \%$ & $-14 \%$ & $-16 \%$ & $-8 \%$ \\
\hline
\end{tabular}

Source: IMF staff estimates based on data from Bank of Mozambique.

1/2009 FSAP update: Liquid assets include cash, deposits with BoM, domestic and foreign short-term interbank deposits and T-Bills. Liquid liabilities include resources of central banks, short-term deposits (of public sector, financial institutions, corporates, public enterprises, individuals, collective organizations and emigrants) and short-term loans from other financial institutions. 2010: Liquid assets include cash and balances at central bank, deposits with credit institutions, investments in credit institutions, financial assets (bonds). Liquid liabilities include demand deposits, resources from central banks and other credit institutions. $2 /$ The liquidity ratio is defined as the ratio of liquid assets to liquid liabilities. 


\title{
INTERNATIONAL DEVELOPMENT ASSOCIATION AND INTERNATIONAL MONETARY FUND
}

\author{
REPUBLIC OF MOZAMBIQUE \\ Joint World Bank/IMF Debt Sustainability Analysis - Update \\ Prepared by the staffs of the International Monetary Fund and \\ the International Development Association \\ Approved by Roger Nord and Dominique Desruelle (IMF) \\ and Marcelo Guigale and Jeffrey Lewis (World Bank)
}

May 24, 2011

This debt sustainability analysis (DSA) updates the joint IMF/IDA DSA from May 27, 2010 reflecting the most recent macroeconomic developments. ${ }^{l}$ Its main results indicate that Mozambique remains at a low risk of debt distress. ${ }^{2}$ In line with the previous DSA, the government's plans to temporarily increase public investment partially financed by external borrowing on nonconcessional terms still calls for caution and resolve to improve debt management capacity. As public debt is largely external, the evolution of public debt indicators mirrors that of external debt.

\section{UNDERLYING DSA ASSUMPTIONS}

1. This DSA is consistent with the macroeconomic framework outlined in the Staff Report for the 2011 Article IV Consultation and Second Review under the Policy Support Instrument. Compared to the previous DSA, ${ }^{3}$ this update includes:

a. A revised macroeconomic framework, including in particular lower fiscal and current account deficits over the medium term. Fiscal performance has surpassed expectations on account of a sustained revenue administration effort, which is likely to continue. The external accounts are expected to benefit from buoyant mega-project

\footnotetext{
${ }^{1}$ In line with the 2010 Staff Guidance Note, a full joint LIC DSA is expected to be prepared once every three years for PRGT-eligible IDA-only countries. In between, short annual updates are expected to be produced unless macroeconomic conditions since the last full DSA have significantly changed. See: "Staff Guidance Note on the Application of the Joint Fund-Bank Debt Sustainability Framework for Low-Income Countries" (IMF/SM/10/16 and IDA/ SecM2010-0029)

${ }^{2}$ The DSA presented in this document is based on the standard low-income countries (LIC) DSA framework. See "Debt Sustainability in Low-Income Countries: Further Considerations on an Operational Framework, Policy Implications" (IMF/SM/05/109 and IDA/SECM2004/0629, 9/10/04)

${ }^{3}$ See Mozambique -- Sixth Review Under the Policy Support Instrument, Second Review Under the Arrangement Under the Exogenous Shocks Facility, and Request for a Three-Year Policy Support Instrument. IMF Country report No. 10/174.
} 
exports, which should more than offset the rising import bill related to surging global food and fuel prices.

b. Updated debt stocks. The current DSA is based on the stock of debt as of end-2010, while the previous one was based on the stock of debt as of end-2009.

c. A revised public investment profile reflecting a slower recourse to nonconcessional external borrowing (NCB) than projected in the past. ${ }^{4}$ This DSA update still assumes the full use of the US\$900 million NCB ceiling over the three-year program, but projects a slightly slower pace of disbursements. Disbursements under the Portuguese credit line have also been updated, in line with current developments.

d. Revised grant financing projections, reflecting a lower grant component in external financing going forward.

\begin{tabular}{|c|c|c|c|c|c|}
\hline \multicolumn{6}{|c|}{ Evolution of selected macroeconomic indicators } \\
\hline & 2009 & 2010 & 2011 & 2012 & 2013 \\
\hline \multicolumn{6}{|c|}{ Real GDP growth (\%) } \\
\hline Previous DSA & 6.3 & 6.5 & 7.5 & 7.6 & 7.9 \\
\hline Current DSA & 6.3 & 6.6 & 7.2 & 7.5 & 7.9 \\
\hline \multicolumn{6}{|c|}{ Nominal GDP (US\$ mill) } \\
\hline Previous DSA & 9.8 & 10.2 & 10.8 & 12.2 & 13.8 \\
\hline Current DSA & 10.1 & 9.8 & 12.1 & 13.5 & 15.1 \\
\hline \multicolumn{6}{|c|}{ Primary fiscal deficit (\% of GDP) } \\
\hline Previous DSA & 5.1 & 4.1 & 6.6 & 6.0 & 5.1 \\
\hline Current DSA & 4.9 & 2.9 & 5.5 & 5.8 & 5.5 \\
\hline \multicolumn{6}{|c|}{ Current account deficit (\% of GDP) } \\
\hline Previous DSA & 11.9 & 13.6 & 12.8 & 13.5 & 13.4 \\
\hline Current DSA & 12.1 & 10.2 & 11.4 & 10.9 & 10.9 \\
\hline \multicolumn{6}{|c|}{ Use of NCB ceiling ( $\%$ of GDP) } \\
\hline Previous DSA & ... & ... & 2.8 & 2.5 & 2.2 \\
\hline Current DSA & $\ldots$ & 0.7 & 2.1 & 2.4 & 2.2 \\
\hline \multicolumn{6}{|c|}{ Disbursements under PTL (\% of GDP) } \\
\hline Previous DSA & $\ldots$ & 0.4 & 1.8 & 1.6 & 1.4 \\
\hline Current DSA & & 1.3 & 1.9 & 1.4 & 1.1 \\
\hline \multicolumn{6}{|c|}{ Grant financing (\% of GDP) $)^{1 /}$} \\
\hline Previous DSA & & . & 11.3 & 11.2 & 11.1 \\
\hline Current DSA & & & 9.3 & 9.0 & 8.9 \\
\hline
\end{tabular}

1/ Grant-equivalent financing includes grants provided directly to the government and through new borrowing (difference between the face value and the PV of new debt).

\footnotetext{
${ }^{4}$ The authorities have signed two nonconcessional loans to date: (i) in December 2010, a loan from China amounting to US\$66 million to modernize Maputo airport; and (ii) in April 2011, a loan from Brazil for the construction of Nacala airport over US\$80 million.
} 


\section{External Debt Sustainability Analysis}

2. Under the baseline scenario, all debt indicators remain well below their respective thresholds, including in the longer term (Table 1a and Figure 1). However, the debt indicators rise significantly towards their respective thresholds over the next five years, as the authorities step up their borrowing to address the country's infrastructure gap. Stress tests reveal that Mozambique's external debt is vulnerable to a one-time depreciation of the exchange rate and an exports shock ${ }^{5}$

\section{The debt sustainability indicators are also sensitive to a recurrence of past} macroeconomic performance. However, basing the analysis solely on historical trends (scenario A1) does not take into account the significant structural changes in the Mozambican economy in the post-civil war period and the considerable improvements in the macroeconomic environment under successive Fund-supported programs, all of which should lessen the likelihood of a repeat of the past economic performance. Among other things, there was a shift in the structure of the economy, as large private capital inflows in the early years of the decade in the natural resources sector subsequently supported a surge in exports and a sharp fall in the current account deficit.

4. In a scenario under which nonconcessional resources are not used productively, external debt indicators would deteriorate, but remain below their respective thresholds. The customized high investment-low growth scenario assumes that foreign financing and related spending would remain unchanged relative to the baseline in nominal terms. However, it assumes a much lower rate of return of the public investment projects compared to the baseline (and therefore a much lower impact on GDP growth over the projection period).

\section{Public Sector Debt Sustainability}

5. The evolution of the public debt indicators (including domestic debt) mirrors that of the external indicators because of the predominance of external debt (Table 2 and Figure 2). The medium-term increase in public debt reflects the temporary surge in public investment financed by external borrowing on nonconcessional terms. However, over the longer term, the public debt stock projections also include a marginal increase in domestic debt from about 3 percent of GDP at end-2010 to 4 percent of GDP in $2031 .^{6}$

\footnotetext{
${ }^{5}$ In last year's DSA, the standard export shock was tailored to capture the historical volatility over the past two decades of prices of aluminum, which accounts for roughly half of Mozambique's export proceeds. The current export shock reflects the same modification.

${ }^{6}$ This should not affect the availability of credit to the private sector.
} 


\section{Conclusions}

6. Mozambique continues to face a low risk of debt distress. Its external debt levels are expected to remain below their indicative thresholds for debt distress. However, the government's plans to temporarily increase public investment financed by external borrowing on nonconcessional terms, will noticeably increase debt vulnerability, as debt ratios under the stress tests approach, and in some instances temporarily and marginally exceed, the relevant thresholds. Although Mozambique's public debt is expected to decline beyond the medium term, stress tests suggest vulnerability, mirroring the large share of external debt in total debt. However, the authorities' commitments under the current PSI, including the continued pursuit of prudent macroeconomic policies and structural reforms to boost their debt management capacity, should be conducive to containing debt vulnerabilities. 
Figure 1. Mozambique: Indicators of Public and Publicly Guaranteed External Debt under Alternatives Scenarios, 2011-2031 1/
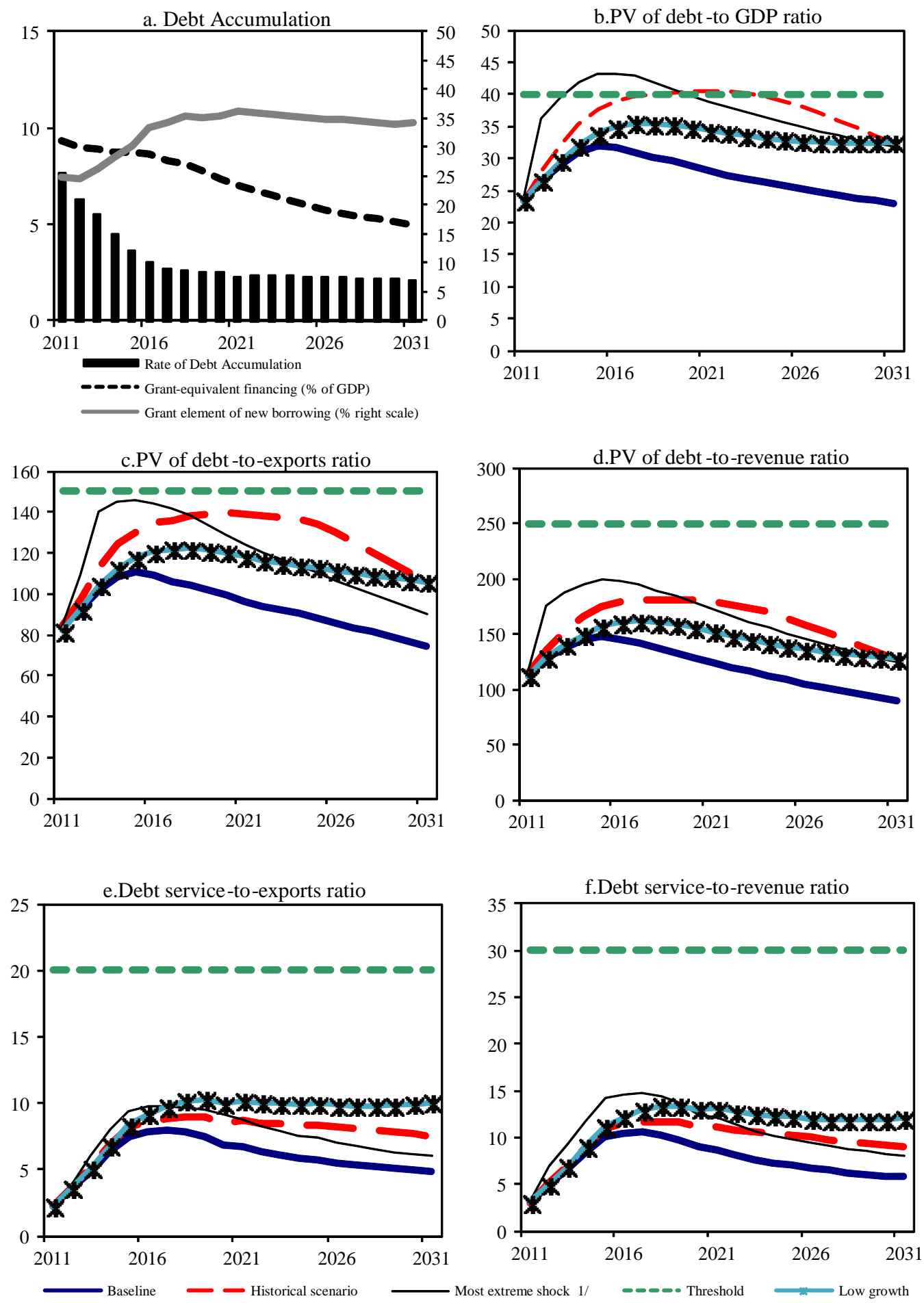

Sources: Country authorities; and staff estimates and projections.

$1 /$ The most extreme stress test is the test that yields the highest ratio in 2021. In figure b. it corresponds to a One-time depreciation shock; in c. to a Exports shock; in d. to a One-time depreciation shock; in e. to a Exports shock and in figure f. to a One-time depreciation shock 
Table 1a.: External Debt Sustainability Framework, Baseline Scenario, 2008-2031 1/ (In percent of GDP, unless otherwise indicated)

\begin{tabular}{|c|c|c|c|c|c|c|c|c|c|c|c|c|c|c|c|}
\hline & \multicolumn{3}{|c|}{ Actual } & \multirow{2}{*}{$\begin{array}{l}\text { Historical } \\
\text { Average }\end{array}$} & \multirow{2}{*}{$\begin{array}{l}0 \text { Standard } \\
0 \text { Deviation }\end{array}$} & \multicolumn{6}{|c|}{ Projections } & \multirow[b]{2}{*}{$\begin{array}{c}2011-2016 \\
\text { Average }\end{array}$} & \multirow[b]{2}{*}{2021} & \multirow{2}{*}{\multicolumn{2}{|c|}{$\begin{aligned} 2017-2031 \\
2031 \quad \text { Average }\end{aligned}$}} \\
\hline & 2008 & 2009 & 2010 & & & 2011 & 2012 & 2013 & 2014 & 2015 & 2016 & & & & \\
\hline External debt (nominal) $1 /$ & 61.5 & 57.5 & 58.0 & & & 54.0 & 54.0 & 54.2 & 54.8 & 55.2 & 54.3 & & 50.7 & 51.8 & \\
\hline $\mathrm{o} / \mathrm{w}$ public and publicly guaranteed $(\mathrm{PPG})$ & 38.0 & 36.5 & 32.5 & & & 35.2 & 38.7 & 41.6 & 44.2 & 45.8 & 45.8 & & 42.3 & 34.9 & \\
\hline Change in external debt & -6.6 & -4.0 & 0.5 & & & -4.0 & 0.0 & 0.2 & 0.6 & 0.4 & -0.9 & & -0.8 & 1.0 & \\
\hline Identified net debt-creating flows & -6.6 & 2.4 & 3.8 & & & 0.8 & 0.2 & 0.6 & -0.1 & 0.0 & -0.2 & & 0.9 & 2.2 & \\
\hline Non-interest current account deficit & 9.5 & 10.6 & 8.6 & 10.4 & 3.3 & 9.8 & 9.4 & 9.3 & 9.1 & 9.1 & 9.1 & & 9.3 & 9.3 & 9.3 \\
\hline Deficit in balance of goods and services & 14.1 & 17.2 & 16.2 & & & 15.8 & 14.5 & 14.2 & 13.8 & 13.5 & 13.3 & & 13.3 & 10.4 & \\
\hline Exports & 32.3 & 27.4 & 29.5 & & & 27.6 & 28.6 & 28.4 & 28.5 & 28.9 & 28.9 & & 29.1 & 30.8 & \\
\hline Imports & 46.4 & 44.5 & 45.7 & & & 43.4 & 43.1 & 42.6 & 42.3 & 42.4 & 42.2 & & 42.4 & 41.2 & \\
\hline Net current transfers (negative $=$ inflow) & -8.6 & -7.6 & -6.8 & -7.4 & 3.0 & -6.2 & -6.0 & -5.9 & -5.7 & -5.7 & -5.4 & & -4.7 & -3.9 & -4.4 \\
\hline $\mathrm{o} / \mathrm{w}$ official & -7.7 & -6.8 & -6.2 & & & -5.6 & -5.3 & -5.2 & -5.1 & -5.0 & -4.7 & & -4.0 & -3.3 & \\
\hline Other current account flows (negative = net inflow) & 4.0 & 1.0 & -0.7 & & & 0.3 & 0.9 & 1.0 & 1.0 & 1.2 & 1.1 & & 0.7 & 2.8 & \\
\hline Net FDI $($ negative $=$ inflow) & -5.9 & -8.8 & -8.1 & -5.8 & 2.5 & -7.2 & -7.1 & -6.5 & -7.0 & -6.7 & -6.8 & & -6.3 & -5.3 & -5.8 \\
\hline Endogenous debt dynamics 2/ & -10.3 & 0.7 & 3.2 & & & -1.8 & -2.1 & -2.2 & -2.2 & -2.4 & -2.4 & & -2.0 & -1.8 & \\
\hline Contribution from nominal interest rate & 2.2 & 1.5 & 1.6 & & & 1.5 & 1.5 & 1.6 & 1.7 & 1.6 & 1.5 & & 1.3 & 1.6 & \\
\hline Contribution from real GDP growth & -3.8 & -3.8 & -3.9 & & & -3.4 & -3.6 & -3.8 & -3.8 & -4.0 & -3.9 & & -3.4 & -3.4 & \\
\hline Contribution from price and exchange rate changes & -8.7 & 3.0 & 5.5 & & & & & & & & & & . & . & \\
\hline Residual (3-4) 3/ & 0.0 & -6.5 & -3.3 & & & -4.8 & -0.2 & -0.3 & 0.6 & 0.5 & -0.7 & & -1.7 & -1.2 & \\
\hline $\mathrm{o} / \mathrm{w}$ exceptional financing & 0.0 & -4.7 & -2.1 & & & -3.3 & 0.0 & 0.0 & 0.0 & 0.0 & 0.0 & & 0.0 & -1.1 & \\
\hline PV of external debt $4 /$ & $\ldots$ & $\ldots$ & 45.7 & & & 42.1 & 41.8 & 41.6 & 41.5 & 41.3 & 40.1 & & 36.4 & 39.9 & \\
\hline In percent of exports & $\ldots$ & $\ldots$ & 154.9 & & & 152.8 & 145.9 & 146.4 & 145.4 & 143.1 & 138.6 & & 125.2 & 129.8 & \\
\hline PV of PPG external debt & ... & ... & 20.2 & & & 23.3 & 26.5 & 29.0 & 30.9 & 31.9 & 31.6 & & 28.0 & 23.0 & \\
\hline In percent of exports & $\ldots$ & $\ldots$ & 68.4 & & & 84.7 & 92.7 & 102.1 & 108.4 & 110.4 & 109.3 & & 96.4 & 74.7 & \\
\hline In percent of government revenues & $\ldots$ & ... & 102.9 & & & 115.5 & 128.7 & 137.2 & 144.3 & 147.9 & 145.0 & & 124.0 & 90.0 & \\
\hline Debt service-to-exports ratio (in percent) & 19.2 & 17.8 & 15.9 & & & 12.6 & 15.5 & 15.7 & 17.6 & 16.9 & 16.9 & & 14.8 & 17.7 & \\
\hline PPG debt service-to-exports ratio (in percent) & 1.6 & 1.7 & 2.3 & & & 2.2 & 3.6 & 4.9 & 6.3 & 7.5 & 7.8 & & 6.7 & 4.8 & \\
\hline PPG debt service-to-revenue ratio (in percent) & 3.1 & 2.6 & 3.4 & & & 3.0 & 4.9 & 6.5 & 8.4 & 10.1 & 10.4 & & 8.6 & 5.8 & \\
\hline Total gross financing need (Billions of U.S. dollars) & 1.0 & 0.7 & 0.5 & & & 0.7 & 0.9 & 1.1 & 1.2 & 1.3 & 1.4 & & 2.4 & 8.8 & \\
\hline Non-interest current account deficit that stabilizes debt ratio & 16.2 & 14.6 & 8.1 & & & 13.8 & 9.5 & 9.1 & 8.5 & 8.6 & 10.0 & & 10.1 & 8.3 & \\
\hline \multicolumn{16}{|l|}{ Key macroeconomic assumptions } \\
\hline Real GDP growth (in percent) & 6.8 & 6.3 & 6.6 & 8.0 & 1.8 & 7.2 & 7.5 & 7.9 & 7.8 & 7.8 & 7.8 & 7.7 & 7.3 & 7.4 & 7.3 \\
\hline GDP deflator in US dollar terms (change in percent) & 14.6 & -4.6 & -8.8 & 1.2 & 9.2 & 15.3 & 3.9 & 3.0 & 1.9 & 0.9 & 1.7 & 4.5 & 3.6 & 3.0 & 3.4 \\
\hline Effective interest rate (percent) $5 /$ & 4.0 & 2.5 & 2.7 & 3.4 & 0.9 & 3.3 & 3.1 & 3.3 & 3.4 & 3.1 & 3.0 & 3.2 & 2.9 & 3.5 & 3.1 \\
\hline Growth of exports of G\&S (US dollar terms, in percent) & 11.8 & -14.0 & 4.9 & 16.4 & 15.6 & 15.4 & 16.0 & 10.2 & 10.4 & 10.1 & 9.9 & 12.0 & 11.3 & 12.4 & 11.4 \\
\hline Growth of imports of G\&S (US dollar terms, in percent) & 25.7 & -2.6 & -0.2 & 12.0 & 11.3 & 17.3 & 11.1 & 9.8 & 9.2 & 9.0 & 9.3 & 10.9 & 10.2 & 11.0 & 10.8 \\
\hline Grant element of new public sector borrowing (in percent) & & & & $\ldots$ & $\ldots$ & 24.8 & 24.6 & 26.2 & 28.2 & 30.4 & 33.5 & 28.0 & 36.2 & 34.3 & 35.0 \\
\hline Government revenues (excluding grants, in percent of GDP) & 15.9 & 17.4 & 19.6 & & & 20.2 & 20.6 & 21.1 & 21.4 & 21.6 & 21.8 & & 22.6 & 25.5 & 23.5 \\
\hline Aid flows (in Billions of US dollars) $6 /$ & 1.2 & 0.9 & 0.8 & & & 1.4 & 1.5 & 1.7 & 1.8 & 2.0 & 2.2 & & 3.0 & 6.3 & \\
\hline $\mathrm{o} / \mathrm{w}$ Grants & 0.9 & 0.9 & 0.8 & & & 0.9 & 1.0 & 1.0 & 1.1 & 1.2 & 1.3 & & 1.8 & 3.5 & \\
\hline $\mathrm{o} / \mathrm{w}$ Concessional loans & 0.2 & 0.0 & 0.0 & & & 0.5 & 0.5 & 0.6 & 0.7 & 0.8 & 0.9 & & 1.2 & 2.8 & \\
\hline Grant-equivalent financing (in percent of GDP) $7 /$ & $\ldots$ & $\ldots$ & $\ldots$ & & & 9.3 & 9.0 & 8.9 & 8.8 & 8.7 & 8.6 & & 7.1 & 5.0 & 6.4 \\
\hline Grant-equivalent financing (in percent of external financing) $7 /$ & $\ldots$ & $\ldots$ & $\ldots$ & & & 60.8 & 60.7 & 62.1 & 64.2 & 66.4 & 68.9 & & 70.2 & 66.6 & 68.4 \\
\hline \multicolumn{16}{|l|}{ Memorandum items: } \\
\hline Nominal GDP (Billions of US dollars) & 9.9 & 10.1 & 9.8 & & & 12.1 & 13.5 & 15.1 & 16.5 & 18.0 & 19.7 & & 32.9 & 94.2 & \\
\hline Nominal dollar GDP growth & 22.4 & 1.4 & -2.8 & & & 23.6 & 11.8 & 11.1 & 9.9 & 8.8 & 9.7 & 12.5 & 11.1 & 10.7 & 11.0 \\
\hline PV of PPG external debt (in Billions of US dollars) & & & 2.0 & & & 2.7 & 3.5 & 4.2 & 4.9 & 5.5 & 6.1 & & 9.1 & 21.4 & \\
\hline (PVt-PVt-1)/GDPt-1 (in percent) & & & & & & 7.6 & 6.3 & 5.5 & 4.5 & 3.6 & 3.0 & 5.1 & 2.3 & 2.1 & 2.3 \\
\hline Gross workers' remittances (Billions of US dollars) & $\ldots$ & $\ldots$ & & & & & & & & & & & & & \\
\hline PV of PPG external debt (in percent of GDP + remittances) & $\ldots$ & $\ldots$ & 20.2 & & & 23.3 & 26.5 & 29.0 & 30.9 & 31.9 & 31.6 & & 28.0 & 23.0 & \\
\hline PV of PPG external debt (in percent of exports + remittances) & $\ldots$ & $\ldots$ & 68.4 & & & 84.7 & 92.7 & 102.1 & 108.4 & 110.4 & 109.3 & & 96.4 & 74.7 & \\
\hline Debt service of PPG external debt (in percent of exports + remittances) & $\ldots$ & $\ldots$ & 2.3 & & & 2.2 & 3.6 & 4.9 & 6.3 & 7.5 & 7.8 & & 6.7 & 4.8 & \\
\hline
\end{tabular}

Sources: Country authorities; and staff estimates and projections.

1/ Includes both public and private sector external debt. Historical averages and standard deviations are generally derived over the past 10 years, subject to data availability.

$2 /$ Derived as $[\mathrm{r}-\mathrm{g}-?(1+\mathrm{g}) /(1+\mathrm{g}+?+\mathrm{g}$ ?) times previous period debt ratio, with $\mathrm{r}=$ nominal interest rate; $\mathrm{g}=$ real GDP growth rate, and $?=$ growth rate of GDP deflator in U.S. dollar terms.

3/ Includes exceptional financing (i.e., changes in arrears and debt relief); changes in gross foreign assets; and valuation adjustments. For projections also includes contribution from price and exchange rate changes.

4/ Assumes that PV of private sector debt is equivalent to its face value.

6/ Defined as grants, concessional loans, and debt relief.

7/ Grant-equivalent financing includes grants provided directly to the government and through new borrowing (difference between the face value and the PV of new debt). 
Table 1b.Mozambique: Sensitivity Analysis for Key Indicators of Public and Publicly Guaranteed External Debt, 2011-2031 (In percent)

\begin{tabular}{|c|c|c|c|c|c|c|c|c|}
\hline & \multicolumn{8}{|c|}{ Projections } \\
\hline & 2011 & 2012 & 2013 & 2014 & 2015 & 2016 & 2021 & 2031 \\
\hline \multicolumn{9}{|c|}{ PV of debt-to GDP ratio } \\
\hline Baseline & 23 & 27 & 29 & 31 & 32 & 32 & 28 & 23 \\
\hline \multicolumn{9}{|l|}{ A. Alternative Scenarios 1/ } \\
\hline A1. Key variables at their historical averages in $2011-20312 /$ & 23 & 28 & 32 & 35 & 38 & 39 & 40 & 32 \\
\hline A3. Alternative Scenario: No Growth Impact of NCB-Financed Investment & 23 & 26 & 30 & 32 & 34 & 35 & 34 & 33 \\
\hline \multicolumn{9}{|l|}{ B. Bound Tests } \\
\hline B1. Real GDP growth at historical average minus one standard deviation in 2012-2013 & 23 & 26 & 29 & 31 & 32 & 32 & 28 & 23 \\
\hline B2. Export value growth at historical average minus one standard deviation in 2012-2013 3/ & 23 & 28 & 34 & 35 & 35 & 35 & 30 & 23 \\
\hline B3. US dollar GDP deflator at historical average minus one standard deviation in 2012-2013 & 23 & 29 & 36 & 38 & 39 & 39 & 35 & 29 \\
\hline B4. Net non-debt creating flows at historical average minus one standard deviation in 2012-2013 4/ & 23 & 30 & 35 & 37 & 37 & 37 & 31 & 24 \\
\hline B5. Combination of B1-B4 using one-half standard deviation shocks & 23 & 32 & 41 & 42 & 43 & 42 & 36 & 27 \\
\hline B6. One-time 30 percent nominal depreciation relative to the baseline in $20125 /$ & 23 & 36 & 40 & 42 & 43 & 43 & 39 & 32 \\
\hline
\end{tabular}

\section{PV of debt-to-exports ratio}

Baseline

A. Alternative Scenarios 1/

A1. Key variables at their historical averages in 2011-2031 2/

A3. Alternative Scenario: No Growth Impact of NCB-Financed Investment

104

112

130

117

\section{B. Bound Tests}

B1. Real GDP growth at historical average minus one standard deviation in 2012-2013

B2. Export value growth at historical average minus one standard deviation in 2012-2013 3/

B3. US dollar GDP deflator at historical average minus one standard deviation in 2012-2013

B4. Net non-debt creating flows at historical average minus one standard deviation in 2012-2013 4/

B5. Combination of B1-B4 using one-half standard deviation shocks

B6. One-time 30 percent nominal depreciation relative to the baseline in 2012 5/

$\begin{array}{rrrrrrrr}85 & 90 & 99 & 104 & 106 & 106 & \mathbf{9 5} & 74 \\ 85 & 109 & 140 & 145 & 146 & 144 & \mathbf{1 2 4} & 90 \\ 85 & 90 & 99 & 104 & 106 & 106 & \mathbf{9 5} & 74 \\ 85 & 104 & 125 & 128 & 129 & 127 & \mathbf{1 0 8} & 77 \\ 85 & 108 & 131 & 134 & 135 & 133 & \mathbf{1 1 4} & 81 \\ 85 & 90 & 99 & 104 & 106 & 106 & \mathbf{9 5} & 74\end{array}$

\section{PV of debt-to-revenue ratio}

Baseline

\section{A. Alternative Scenarios 1/}

A1. Key variables at their historical averages in 2011-2031 2/

A3. Alternative Scenario: No Growth Impact of NCB-Financed Investment

\section{B. Bound Tests}

B1. Real GDP growth at historical average minus one standard deviation in 2012-2013

B2. Export value growth at historical average minus one standard deviation in 2012-2013 3/

B3. US dollar GDP deflator at historical average minus one standard deviation in 2012-2013

B4. Net non-debt creating flows at historical average minus one standard deviation in 2012-2013 4/

B5. Combination of B1-B4 using one-half standard deviation shocks

B6. One-time 30 percent nominal depreciation relative to the baseline in 20125 /

137
159
169
168
193
187

143
162
175
171
197
195

143
162
175
171
197
195


Table 1b. Mozambique: Sensitivity Analysis for Key Indicators of Public and Publicly Guaranteed External Debt, 2011-2031 (continued) (In percent)

\section{Debt service-to-exports ratio}

\section{Baseline}

\section{A. Alternative Scenarios 1/}

A1. Key variables at their historical averages in 2011-2031 2/

A3. Alternative Scenario: No Growth Impact of NCB-Financed Investment

\section{B. Bound Tests}

B1. Real GDP growth at historical average minus one standard deviation in 2012-2013 B2. Export value growth at historical average minus one standard deviation in 2012-2013 3/

B3. US dollar GDP deflator at historical average minus one standard deviation in 2012-2013 B4. Net non-debt creating flows at historical average minus one standard deviation in 2012-2013 4/

B5. Combination of B1-B4 using one-half standard deviation shocks

B6. One-time 30 percent nominal depreciation relative to the baseline in 20125 /

4

5

6

8

$\begin{array}{lll}8 & 7 & 5\end{array}$

(1)

$\begin{array}{rrrrrrrr}2 & 4 & 5 & 7 & 8 & 9 & 9 & 7 \\ 2 & 4 & 5 & 7 & 8 & 9 & 10 & 10\end{array}$

\section{Debt service-to-revenue ratio}

Baseline

\section{A. Alternative Scenarios 1/}

A1. Key variables at their historical averages in 2011-2031 2/

A3. Alternative Scenario: No Growth Impact of NCB-Financed Investment

$\begin{array}{ll}2 & 4 \\ 2 & 4 \\ 2 & 4 \\ 2 & 4 \\ 2 & 4 \\ 2 & 4\end{array}$

\section{B. Bound Tests}

B1. Real GDP growth at historical average minus one standard deviation in 2012-2013

B2. Export value growth at historical average minus one standard deviation in 2012-2013 3/

B3. US dollar GDP deflator at historical average minus one standard deviation in 2012-2013

B4. Net non-debt creating flows at historical average minus one standard deviation in 2012-2013 4/

B5. Combination of B1-B4 using one-half standard deviation shocks

B6. One-time 30 percent nominal depreciation relative to the baseline in 2012 5/

Memorandum item:

Grant element assumed on residual financing (i.e., financing required above baseline)

$\begin{array}{rrrrrrrr}3 & 5 & 7 & 9 & 10 & 11 & \mathbf{9} & 6 \\ 3 & 5 & 7 & 9 & 11 & 11 & \mathbf{1 0} & 6 \\ 3 & 6 & 8 & 11 & 13 & 13 & \mathbf{1 1} & 7 \\ 3 & 5 & 7 & 9 & 11 & 11 & \mathbf{1 0} & 6 \\ 3 & 5 & 8 & 11 & 13 & 13 & \mathbf{1 1} & 7 \\ 3 & 7 & 9 & 12 & 14 & 15 & \mathbf{1 2} & 8 \\ & & & & & & & \\ 25 & 25 & 25 & 25 & 25 & 25 & \mathbf{2 5} & 25\end{array}$

Sources: Country authorities; and staff estimates and projections.

1/ The stress test A2 (borrowing in less concessional terms) has not been included in this case (in line with the 2010 DSA). Given the commercial financing terms already included in theseline for the investment projects, this scenario would yield unrealistic results, as marginal borrowing under this shock is calculated on the average terms of new borrowing; therefore assuming harsher terms than Mozambique would actually face in the need to cover a financing gap.

2/ Variables include real GDP growth, growth of GDP deflator (in U.S. dollar terms), non-interest current account in percent of GDP, and non-debt creating flows.

3/ Exports values are assumed to remain permanently at the lower level, but the current account as a share of GDP is assumed to return to its baseline level after the shock (implicitly assoffigatging adjustment in import levels).

4/ Includes official and private transfers and FDI.

5/ Depreciation is defined as percentage decline in dollar/local currency rate, such that it never exceeds 100 percent. 
Figure 2.Mozambique: Indicators of Public Debt Under Alternative Scenarios, 2011-2031 1/
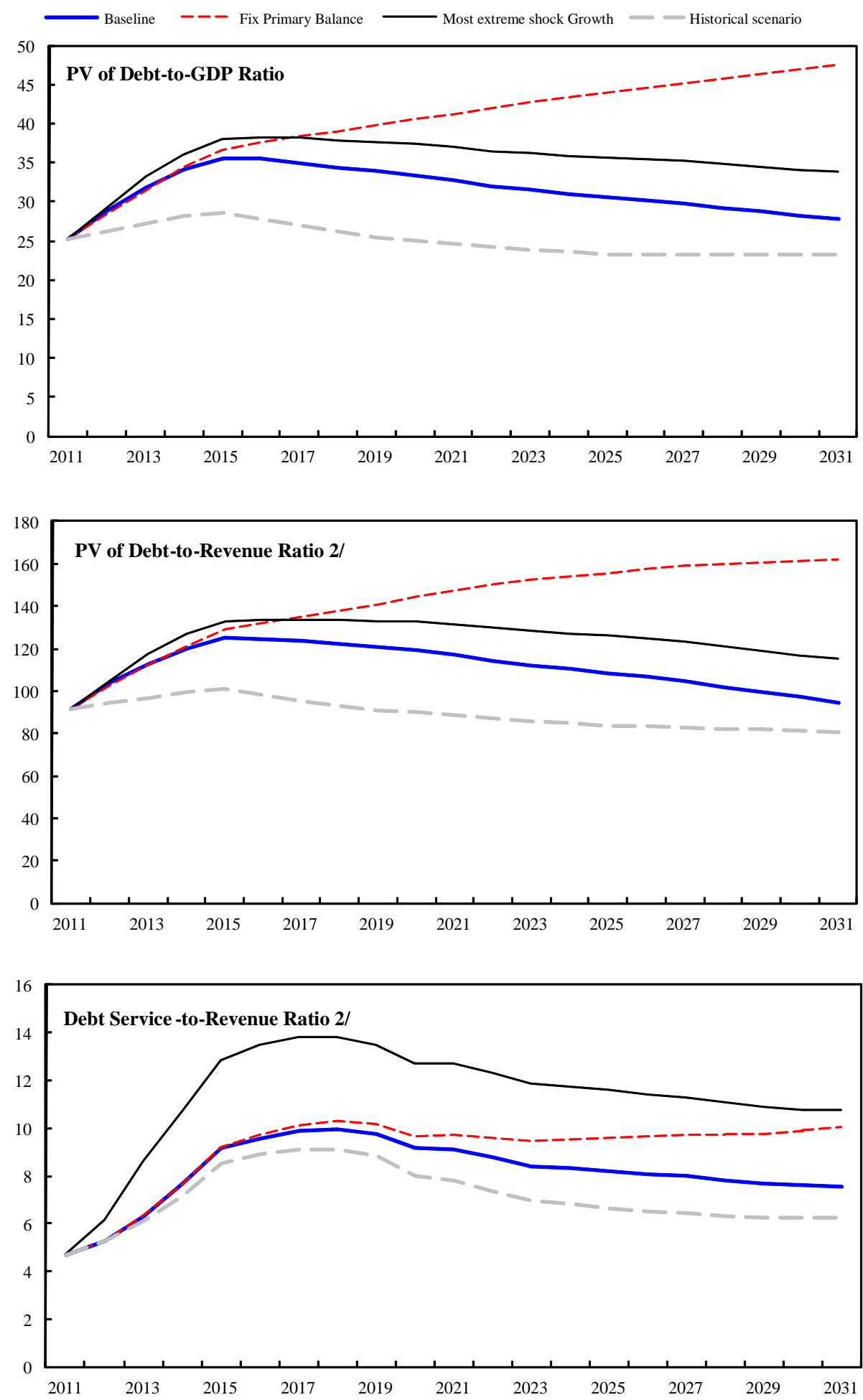

Sources: Country authorities; and staff estimates and projections.

$1 /$ The most extreme stress test is the test that yields the highest ratio in 2021.

2/ Revenues are defined inclusive of grants. 
Table 2a.Mozambique: Public Sector Debt Sustainability Framework, Baseline Scenario, 2008-2031

(In percent of GDP, unless otherwise indicated)

\begin{tabular}{|c|c|c|c|c|c|c|c|c|c|c|c|c|c|c|c|}
\hline & \multicolumn{3}{|c|}{ Actual } & \multirow[b]{2}{*}{ Average } & \multirow[b]{2}{*}{$\begin{array}{l}\text { Standard } \\
\text { Deviation } \\
\end{array}$} & \multicolumn{5}{|l|}{ Estimate } & \multicolumn{5}{|c|}{ Projections } \\
\hline & 2008 & 2009 & 2010 & & & 2011 & 2012 & 2013 & 2014 & 2015 & 2016 & $\begin{array}{c}2011-16 \\
\text { Average }\end{array}$ & 2021 & 2031 & $\begin{array}{r}2017-31 \\
\text { Average } \\
\end{array}$ \\
\hline $\begin{array}{l}\text { Public sector debt 1/ } \\
\text { o/w foreign-currency denominated }\end{array}$ & $\begin{array}{l}42.1 \\
38.0\end{array}$ & $\begin{array}{l}39.7 \\
36.5\end{array}$ & $\begin{array}{l}35.3 \\
32.5\end{array}$ & & & $\begin{array}{l}37.2 \\
35.2\end{array}$ & $\begin{array}{l}40.9 \\
38.7\end{array}$ & $\begin{array}{l}44.3 \\
41.6\end{array}$ & $\begin{array}{l}47.4 \\
44.2\end{array}$ & $\begin{array}{l}49.3 \\
45.8\end{array}$ & $\begin{array}{l}49.6 \\
45.8\end{array}$ & & $\begin{array}{l}46.9 \\
42.3\end{array}$ & $\begin{array}{l}39.6 \\
34.9\end{array}$ & \\
\hline Change in public sector debt & 0.2 & -2.5 & -4.4 & & & 1.9 & 3.7 & 3.4 & 3.0 & 2.0 & 0.3 & & -0.9 & -0.7 & \\
\hline Identified debt-creating flows & -5.2 & 3.9 & 2.8 & & & 0.8 & 2.6 & 2.3 & 2.2 & 1.4 & 0.4 & & -0.8 & -0.5 & \\
\hline Primary deficit & -1.6 & 4.9 & 2.9 & 2.6 & 1.8 & 5.5 & 5.8 & 5.5 & 4.8 & 4.2 & 4.1 & 5.0 & 2.8 & 2.3 & 2.8 \\
\hline Revenue and grants & 25.3 & 26.7 & 27.7 & & & 27.6 & 27.7 & 28.1 & 28.3 & 28.4 & 28.5 & & 28.0 & 29.2 & \\
\hline of which: grants & 9.4 & 9.4 & 8.1 & & & 7.3 & 7.1 & 7.0 & 6.9 & 6.8 & 6.7 & & 5.4 & 3.7 & \\
\hline Primary (noninterest) expenditure & 23.8 & 31.7 & 30.6 & & & 33.0 & 33.5 & 33.5 & 33.1 & 32.6 & 32.5 & & 30.8 & 31.5 & \\
\hline Automatic debt dynamics & -3.4 & -0.8 & 0.0 & & & -4.7 & -3.2 & -3.2 & -2.6 & -2.8 & -3.6 & & -3.6 & -2.8 & \\
\hline Contribution from interest rate/growth differential & -6.9 & -0.5 & 1.3 & & & -5.7 & -3.0 & -3.2 & -3.0 & -2.8 & -3.2 & & -3.6 & -2.8 & \\
\hline of which: contribution from average real interest rate & -4.3 & 2.0 & 3.8 & & & -3.3 & -0.4 & -0.2 & 0.2 & 0.7 & 0.4 & & -0.4 & 0.0 & \\
\hline of which: contribution from real GDP growth & -2.7 & -2.5 & -2.5 & & & -2.4 & -2.6 & -3.0 & -3.2 & -3.4 & -3.6 & & -3.2 & -2.8 & \\
\hline Contribution from real exchange rate depreciation & 3.6 & -0.3 & -1.3 & & & 1.0 & -0.2 & 0.0 & 0.4 & 0.0 & -0.4 & & $\ldots$ & $\ldots$ & \\
\hline Other identified debt-creating flows & -0.3 & -0.3 & -0.2 & & & 0.0 & 0.0 & 0.0 & 0.0 & 0.0 & 0.0 & & 0.0 & 0.0 & \\
\hline Privatization receipts (negative) & 0.0 & -0.2 & -0.1 & & & 0.0 & 0.0 & 0.0 & 0.0 & 0.0 & 0.0 & & 0.0 & 0.0 & \\
\hline Recognition of implicit or contingent liabilities & 0.0 & 0.0 & 0.0 & & & 0.0 & 0.0 & 0.0 & 0.0 & 0.0 & 0.0 & & 0.0 & 0.0 & \\
\hline Debt relief (HIPC and other) & -0.3 & -0.1 & -0.1 & & & 0.0 & 0.0 & 0.0 & 0.0 & 0.0 & 0.0 & & 0.0 & 0.0 & \\
\hline Other (specify, e.g. bank recapitalization) & 0.0 & 0.0 & 0.0 & & & 0.0 & 0.0 & 0.0 & 0.0 & 0.0 & 0.0 & & 0.0 & 0.0 & \\
\hline Residual, including asset changes & 5.5 & -6.4 & -7.1 & & & 1.1 & 1.1 & 1.2 & 0.8 & 0.5 & -0.1 & & 0.0 & -0.2 & \\
\hline \multicolumn{16}{|l|}{ Other Sustainability Indicators } \\
\hline PV of public sector debt & $\ldots$ & $\ldots$ & 23.0 & & & 25.3 & 28.7 & 31.7 & 34.1 & 35.4 & 35.4 & & 32.7 & 27.7 & \\
\hline $\mathrm{o} / \mathrm{w}$ foreign-currency denominated & $\ldots$ & $\ldots$ & 20.2 & & & 23.3 & 26.5 & 29.0 & 30.9 & 31.9 & 31.6 & & 28.0 & 23.0 & \\
\hline $\mathrm{o} / \mathrm{w}$ external & $\ldots$ & $\ldots$ & 20.2 & & & 23.3 & 26.5 & 29.0 & 30.9 & 31.9 & 31.6 & & 28.0 & 23.0 & \\
\hline $\mathrm{PV}$ of contingent liabilities (not included in public sector debt) & $\ldots$ & $\ldots$ & $\ldots$ & & & $\ldots$ & $\ldots$ & $\ldots$ & $\ldots$ & $\ldots$ & $\ldots$ & & $\ldots$ & $\ldots$ & \\
\hline Gross financing need $2 /$ & -0.4 & 5.9 & 4.4 & & & 6.8 & 7.3 & 7.2 & 6.9 & 6.8 & 6.8 & & 5.4 & 4.5 & \\
\hline $\mathrm{PV}$ of public sector debt-to-revenue and grants ratio (in percent) & $\ldots$ & $\ldots$ & 83.1 & & & 91.8 & 103.6 & 112.8 & 120.4 & 125.0 & 124.4 & & 116.8 & 94.7 & \\
\hline $\mathrm{PV}$ of public sector debt-to-revenue ratio (in percent) & $\ldots$ & $\ldots$ & 117.3 & & & 125.1 & 139.2 & 150.0 & 158.9 & 164.3 & 162.5 & & 144.4 & 108.5 & \\
\hline $\mathrm{o} / \mathrm{w}$ external $3 /$ & $\ldots$ & $\ldots$ & 102.9 & & & 115.5 & 128.7 & 137.2 & 144.3 & 147.9 & 145.0 & & 124.0 & 90.0 & \\
\hline Debt service-to-revenue and grants ratio (in percent) $4 /$ & 4.8 & 3.5 & 5.3 & & & 4.7 & 5.3 & 6.3 & 7.7 & 9.2 & 9.6 & & 9.1 & 7.6 & \\
\hline Debt service-to-revenue ratio (in percent) $4 /$ & 7.6 & 5.4 & 7.5 & & & 6.4 & 7.1 & 8.4 & 10.1 & 12.0 & 12.5 & & 11.3 & 8.7 & \\
\hline Primary deficit that stabilizes the debt-to-GDP ratio & -1.8 & 7.4 & 7.3 & & & 3.6 & 2.1 & 2.0 & 1.8 & 2.2 & 3.8 & & 3.7 & 3.0 & \\
\hline \multicolumn{16}{|l|}{ Key macroeconomic and fiscal assumptions } \\
\hline Real GDP growth (in percent) & 6.8 & 6.3 & 6.6 & 8.0 & 1.8 & 7.2 & 7.5 & 7.9 & 7.8 & 7.8 & 7.8 & 7.7 & 7.3 & 7.4 & 7.3 \\
\hline Average nominal interest rate on forex debt (in percent) & 0.7 & 0.6 & 0.6 & 0.8 & 0.6 & 1.2 & 1.6 & 1.8 & 1.9 & 1.9 & 1.9 & 1.7 & 1.6 & 1.5 & 1.6 \\
\hline Average real interest rate on domestic debt (in percent) & 1.5 & 2.7 & 7.8 & 12.2 & 16.2 & 14.2 & 16.9 & 9.9 & 8.9 & 8.9 & 8.5 & 11.2 & 8.5 & 11.4 & 9.3 \\
\hline Real exchange rate depreciation (in percent, + indicates depreciation) & 11.4 & -0.8 & -3.4 & -0.3 & 11.9 & 3.8 & $\ldots$ & 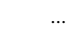 & $\ldots$ & $\ldots$ & $\ldots$ & $\ldots$ & $\ldots$ & $\ldots$ & $\ldots$ \\
\hline Inflation rate (GDP deflator, in percent) & 8.4 & 5.4 & 12.6 & 8.7 & 3.0 & 9.6 & 7.5 & 6.0 & 5.9 & 5.9 & 5.8 & 6.8 & 5.7 & 5.1 & 5.7 \\
\hline Growth of real primary spending (deflated by GDP deflator, in percent) & -0.1 & 0.4 & 0.0 & 0.1 & 0.2 & 0.2 & 0.1 & 0.1 & 0.1 & 0.1 & 0.1 & 0.1 & 0.1 & 0.1 & 0.1 \\
\hline Grant element of new external borrowing (in percent) & $\ldots$ & $\ldots$ & $\ldots$ & $\ldots$ & $\ldots$ & 24.8 & 24.6 & 26.2 & 28.2 & 30.4 & 33.5 & 28.0 & 36.2 & 34.3 & \\
\hline
\end{tabular}

Sources: Country authorities; and staff estimates and projections.

1/ Includes central government external PPG debt and net domestic debt. Historical averages and standard deviations are generally derived over the past 10 years, subject to data availability

$2 /$ Gross financing need is defined as the primary deficit plus debt service plus the stock of short-term debt at the end of the last period.

3/ Revenues excluding grants.

4/Debt service is defined as the sum of interest and amortization of medium and long-term debt. 
Table 2b.Mozambique: Sensitivity Analysis for Key Indicators of Public Debt 2011-2031

\begin{tabular}{l}
\hline PV of Debt-to-GDP Ratio \\
\hline Baseline
\end{tabular}

\section{Baseline}

\section{A. Alternative scenarios}

A1. Real GDP growth and primary balance are at historical averages

A2. Primary balance is unchanged from 2011

A3. Permanently lower GDP growth $1 /$

\section{B. Bound tests}

B1. Real GDP growth is at historical average minus one standard deviations in 2012-2013

B2. Primary balance is at historical average minus one standard deviations in 2012-2013

B3. Combination of B1-B2 using one half standard deviation shocks

B4. One-time 30 percent real depreciation in 2012

B5. 10 percent of GDP increase in other debt-creating flows in 2012

Projections

\begin{tabular}{llllllll}
2011 & 2012 & 2013 & 2014 & 2015 & 2016 & 2021 & 2031 \\
\hline
\end{tabular}

$\begin{array}{llllllll}25 & 29 & 32 & 34 & 35 & 35 & 33 & 28 \\ & & & & & & & \\ 25 & 26 & 27 & 28 & 29 & 28 & 25 & 23 \\ 25 & 28 & 31 & 34 & 37 & 38 & 41 & 47 \\ 25 & 29 & 32 & 35 & 37 & 37 & 37 & 39\end{array}$

PV of Debt-to-Revenue Ratio 2/

\section{Baseline}

$\begin{array}{llllllll}25 & 29 & 33 & 36 & 38 & 38 & 37 & 34 \\ 25 & 28 & 30 & 32 & 34 & 34 & 32 & 27 \\ 25 & 27 & 29 & 32 & 33 & 34 & 32 & 29 \\ 25 & 38 & 39 & 41 & 41 & 40 & 35 & 28 \\ 25 & 36 & 39 & 41 & 42 & 41 & 37 & 29\end{array}$

\section{A. Alternative scenarios}

A1. Real GDP growth and primary balance are at historical averages

A2. Primary balance is unchanged from 2011

A3. Permanently lower GDP growth $1 /$

$\begin{array}{rrrrrrrr}92 & 95 & 97 & 100 & 101 & 98 & 88 & 81 \\ 92 & 103 & 112 & 121 & 129 & 132 & 148 & 162 \\ 92 & 104 & 114 & 123 & 128 & 129 & 130 & 133\end{array}$

\section{B. Bound tests}

B1. Real GDP growth is at historical average minus one standard deviations in 2012-2013

B2. Primary balance is at historical average minus one standard deviations in 2012-2013

B3. Combination of B1-B2 using one half standard deviation shocks

B4. One-time 30 percent real depreciation in 2012

B5. 10 percent of GDP increase in other debt-creating flows in 2012

\section{Debt Service-to-Revenue Ratio 2/}

\section{Baseline}

$\begin{array}{rrrrrrrr}92 & 105 & 117 & 127 & 133 & 133 & 131 & 115 \\ 92 & 100 & 106 & 114 & 119 & 119 & 113 & 93 \\ 92 & 98 & 103 & 111 & 117 & 118 & 116 & 100 \\ 92 & 136 & 139 & 143 & 145 & 142 & 124 & 97 \\ 92 & 130 & 137 & 143 & 147 & 145 & 131 & 101\end{array}$

\section{A. Alternative scenarios}

A1. Real GDP growth and primary balance are at historical averages

A2. Primary balance is unchanged from 2011

A3. Permanently lower GDP growth $1 /$

$\begin{array}{rrrrrrrr}5 & 5 & 6 & 7 & 9 & 9 & 8 & 6 \\ 5 & 5 & 6 & 8 & 9 & 10 & 10 & 10 \\ 5 & 5 & 6 & 8 & 9 & 10 & 10 & 9\end{array}$

\section{B. Bound tests}

B1. Real GDP growth is at historical average minus one standard deviations in 2012-2013

B2. Primary balance is at historical average minus one standard deviations in 2012-2013

B3. Combination of B1-B2 using one half standard deviation shocks

B4. One-time 30 percent real depreciation in 2012

B5. 10 percent of GDP increase in other debt-creating flows in 2012

$\begin{array}{rrrrrrrr}5 & 5 & 6 & 8 & 9 & 10 & 10 & 8 \\ 5 & 5 & 6 & 7 & 9 & 9 & 9 & 7 \\ 5 & 5 & 6 & 7 & 9 & 9 & 9 & 8 \\ 5 & 6 & 9 & 11 & 13 & 13 & 13 & 11 \\ 5 & 5 & 7 & 9 & 10 & 10 & 10 & 8\end{array}$

Sources: Country authorities; and staff estimates and projections.

1/ Assumes that real GDP growth is at baseline minus one standard deviation divided by the square root of the length of the projection period.

2/ Revenues are defined inclusive of grants. 


\title{
INTERNATIONAL MONETARY FUND \\ REPUBLIC OF MOZAMBIQUE
}

\section{Article IV Consultation, Second Review Under the Policy Support Instrument and Request for Modification of Assessment Criteria- Informational Annex}

\author{
Prepared by the African Department \\ (In consultation with other departments)
}

May 24, 2011

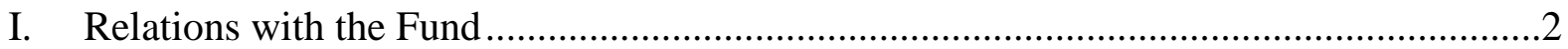

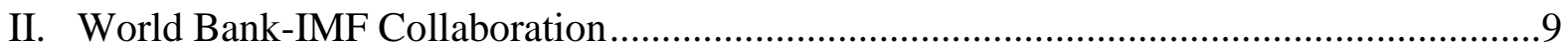

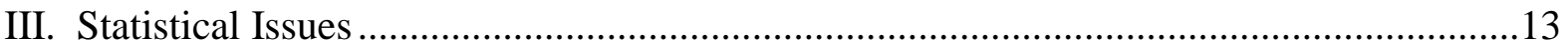




\section{APPENDIX I. MOZAMBIQUE: RELATIONS WITH THE FUND}

(as of April 30, 2011)

I. Membership Status ${ }^{1}$ : Joined: September 24, 1984;

Article VIII

II. General Resources Account:

SDR Million

113.60

\% Quota

Quota

113.60

100.00

Fund holdings of currency

0.01

100.00

Reserve Position

SDR Million

108.84

108.16

$\underline{\text { Holdings }}$

IV. Outstanding Purchases and Loans:

ESF Arrangements

ECF Arrangements
SDR Million

113.60

9.25
\%Allocation

100.00

99.37

\%Quota

100.00

8.15

\section{Latest Financial Arrangements:}

\begin{tabular}{lllrr}
\hline Type & \multicolumn{1}{c}{$\begin{array}{c}\text { Date of } \\
\text { Arrangement }\end{array}$} & $\begin{array}{c}\text { Expiration } \\
\text { Date }\end{array}$ & $\begin{array}{c}\text { Amount Approved } \\
\text { (SDR Million) }\end{array}$ & $\begin{array}{r}\text { Amount Drawn } \\
\text { (SDR Million) }\end{array}$ \\
ESF $^{2}$ & Jun 30, 2009 & Jun 28, 2010 & 113.60 & 113.60 \\
ECF $^{2}$ & Jul 06, 2004 & Jul 05, 2007 & 11.36 & 11.36 \\
ECF $^{2}$ & Jun 28, 1999 & Jun 28, 2003 & 87.20 & 78.80
\end{tabular}

VI. Projected Payments to Fund ${ }^{3}$

(SDR Million; based on existing use of resources and present holdings of SDRs):

\begin{tabular}{|c|c|c|c|c|c|}
\hline & \multicolumn{4}{|c|}{ Forthcoming } & \\
\hline & 2011 & 2012 & $\underline{2013}$ & $\underline{2014}$ & 2015 \\
\hline Principal & 0.65 & 1.46 & 1.95 & 1.95 & 23.09 \\
\hline Charges/Interest & $\underline{0.00}$ & $\underline{0.31}$ & $\underline{0.30}$ & $\underline{0.30}$ & $\underline{0.26}$ \\
\hline Total & $\overline{0.65}$ & $\overline{1.77}$ & 2.25 & 2.25 & 23.35 \\
\hline
\end{tabular}

\footnotetext{
${ }^{1}$ On May 20, 2011, the authorities accepted the obligations under Article VIII of the Fund's Articles of Agreement.

${ }^{2}$ Former PRGF.

${ }^{3}$ When a member has overdue financial obligations outstanding for more than three months, the amount of such arrears will be shown in this section.
} 
VII. Implementation of HIPC Initiative:

I. Commitment of HIPC assistance

Decision point date

Assistance committed

by all creditors (US\$ Million) ${ }^{4}$

Of which: IMF assistance (US\$ million)

(SDR equivalent in millions)

Original Enhanced

Framework Framework

Apr 1998 Apr 2000

$1,716.00 \quad 306.00$

$124.60 \quad 18.46$

$93.17 \quad 13.73$

Completion point date

Jun. 1999 Sep. 2001

II. Disbursement of IMF assistance (SDR Million)

Assistance disbursed to the member

93.17

13.73

106.90

Interim assistance

2.31

2.31

Completion point balance

93.17

11.42

104.58

Additional disbursement of interest income ${ }^{5}$

Total disbursements

$93.17 \quad 14.83$

1.10

1.10

108.00

\section{Implementation of Multilateral Debt Relief Initiative (MDRI):}

I. MDRI-eligible debt (SDR Million) ${ }^{6}$

106.56

Financed by: MDRI Trust

83.05

Remaining HIPC resources

23.51

II. Debt Relief by Facility (SDR Million)

Eligible Debt

$\begin{array}{lrrr}\frac{\text { Delivery Date }}{\text { January } 2006} & \underline{\text { GRA }} & \underline{\text { PRGF }} & \underline{\text { Total }} \\ \text { N/A } & 106.56 & 106.56\end{array}$

\footnotetext{
${ }^{4}$ Assistance committed under the original framework is expressed in net present value (NPV) terms at the completion point, and assistance committed under the enhanced framework is expressed in NPV terms at the decision point. Hence these two amounts can not be added.

${ }^{5}$ Under the enhanced framework, an additional disbursement is made at the completion point corresponding to interest income earned on the amount committed at the decision point but not disbursed during the interim period.

${ }^{6}$ The MDRI provides 100 percent debt relief to eligible member countries qualified for the assistance. Grant assistance from the MDRI Trust and HIPC resources provide debt relief to cover the full stock of debt owed to the Fund as of end-2004 outstanding at the time the member qualifies for debt relief.
} 


\section{Safeguards assessment}

An update safeguards assessment of the Bank of Mozambique (BM) was completed on January 11, 2010 for the ESF. The assessment found improvements in financial reporting practices following the implementation of IFRS. It noted vulnerabilities in the oversight mechanism for external and internal auditing, controls, financial reporting, and in the BM's legal structure. It recommended that the BM commission an external quality assurance review of the internal audit function. Staff will follow-up on progress in implementing the assessment's recommendations.

\section{Exchange arrangement}

Mozambique has a de jure and de facto floating exchange rate arrangement. The exchange rate is largely determined in the interbank foreign exchange market (MCI). The BM regularly intervenes in the market to smooth seasonal fluctuations, provide foreign exchange liquidity received by the government in the form of aid, and sterilize domestic liquidity as part of its monetary policy operations.

On May 20, 2011, Mozambique accepted its obligations under Article VIII, Sections 2, 3, and 4 of the Fund's Articles of Agreement. This process benefited from an Article VIII mission by LEG and PDR in March 2004, a follow-up mission to conduct the jurisdictional review of the foreign exchange system led by LEG and MCM in September 2010, and a number of video-conferences and bilateral exchanges between the authorities, and LEG and MCM from October 2010 to May 2011. A new foreign exchange law-"Foreign Exchange Law" Law No. 11/2009 — came into effect on March 11, 2009. A new foreign exchange regulation to implement the foreign exchange law - the "Regulation for the Foreign Exchange Law" (the "Regulation") (Decreto No. 83/2010)—was issued on December 31, 2010. The Regulation, in conjunction with the implementing norms to be issued shortly by the BM, is intended to fully remove the existing exchange restrictions subject to Article VIII, Sections 2, 3, and 4 of the Fund's Articles of Agreement arising from: (i) the discretionary prior approval for remittances of family living expenses; (ii) the authorization for the purchase of foreign exchange in excess of US $\$ 5,000$ for certain transactions; (iii) the prohibition of advance payments for the import of goods; (iv) the prohibition of advance payments for services; and (v) the prohibition of the conversion of balances of nonresidents' domestic currency accounts into foreign currency or transfer abroad.

The 2010 LEG/MCM mission also identified two multiple currency practices (MCP). The MCPs arise from: (i) potential deviations between the rate used by the Bank of Mozambique in its transactions with the government and the World Bank and the exchange rate in the interbank market (MCI); and (ii) the multiple price foreign exchange auction system. With respect to the MCP arising from potential deviations between the rate used by the BM in its transactions with the government and the World Bank, on March 30, 2011, the BM adopted a formal decision to limit potential deviations between the exchange rates in the interbank market and the exchange rate used by the BM in its foreign exchange transactions with the government and the World Bank, with a view of eliminating the MCP. The notifications to the affected parties will be issued in due course, and the new practice will be implemented as soon as the necessary technical conditions will be in place. The MCP arising from the multiple price foreign exchange auction system has been removed effective April 27, 2011 through the adoption of a new regulation on the interbank exchange market (Aviso 
$\mathrm{n}^{\circ}$ 2/GBM/2011), temporarily discontinuing the previous multiple price auction arrangement, which in any case had not been used since 2009 .

\begin{abstract}
AML-CFT framework
A Financial Intelligence Unit (GIFIM) is in the process of being made fully operational, while the amendment of the AML/CFT law is still awaiting parliamentary approval. Upon its approval, the BM will issue guidelines that would allow Mozambique to move closer toward compliance with the 40+9-Recommendations of the Financial Action Task Force (FATF). In order to ensure a consistent application of the AML/CFT framework across various regulators, the Government intends to establish, and appoint the members of, the National AML Task Force by end-September 2011. Mozambique's first AML/CFT Mutual Evaluation Report by the Eastern and Southern Africa Anti-Money Laundering Group (ESAAMLG) has been considered by the ESAAMLG Plenary in April 2011. The FATF's International Cooperation Review Group (ICRG) is expected to conduct a preliminary review of Mozambique's evaluation report in October. The ICRG process is designed to publicly identify high-risk jurisdictions, and could result in a public statement by the FATF to apply counter-measures against non-cooperative jurisdictions.
\end{abstract}

\title{
Article IV consultation
}

In accordance with Decision No 12794-(02/76), as amended by Decision No 12854-(02/96), Mozambique is on a 24-month Article IV cycle due to the approval of a PSI in June 2007. The 2009 Article IV consultation was completed by the Executive Board on June 30, 2009 (Country Report No. 09/227).

In concluding the 2009 Article IV consultation, Executive Directors welcomed Mozambique's continued strong macroeconomic performance in 2008 despite a challenging external environment. They commended the authorities' flexible policy response to higher fuel and food import prices, which helped to mitigate the impact of these shocks. Directors noted that Mozambique remains vulnerable to the global economic slowdown and agreed that the country's strong track record of prudent macroeconomic policy implementation has provided room to ease fiscal and monetary policy in the near term. In the medium term, Directors underscored the importance of preserving macroeconomic stability and supported efforts to enhance domestic revenue mobilization and to improve public financial management. They welcomed the authorities' intention to limit recourse to domestic financing of the budget so as to preserve sufficient room for private sector credit growth. With respect to the overvaluation of the exchange rate in real effective terms, Directors suggested paying closer attention to a basket of currencies, which could help support Mozambique's competitiveness and economic diversification. Further progress in structural reforms and in developing and safeguarding the soundness of the banking system were seen as instrumental in supporting the business environment.

\section{Ex post assessment of performance under Fund-supported programs}

An ex post assessment (EPA) of Mozambique's performance under Fund-Supported Programs since 1987 was undertaken in December 2003 under the guidelines on assessments of countries with a longer-term program engagement. The EPA was circulated to the Executive Board on November 21, 2003 (Country Report No. 04/53). 
Directors commended the authorities for their pursuit of sound macroeconomic policies and wide ranging structural reforms over the past fifteen years which contributed to strong growth of the economy, and a steady decline in poverty rates. Noting that despite considerable progress Mozambique remains a very poor and vulnerable country, Directors urged the authorities to persevere in their efforts to consolidate macroeconomic stability and accelerate and deepen structural reforms with a view to sustaining economic growth, encouraging employment creation, and further reducing poverty.

\section{FSAP participation and ROSCs}

A Financial Sector Assessment Program (FSAP) for Mozambique was undertaken during the first quarter of 2003. The related Financial Sector Stability Assessment was circulated to the Executive Board on November 19, 2003 (Country Report No. 04/52). An update to the FSAP took place in February 2009 and the related Financial Sector Stability Assessment circulated to the Board on June 19, 2009 (Country Report No. 10/12) A ROSC on fiscal transparency was issued on February 22, 2001. This ROSC was updated in the context of the 2002 Article IV consultation (Country Report No. 02/140) and the 2003 Article IV Consultation (Country Report No. 04/50). The ROSC on fiscal transparency was updated in May 2008. A Report on the Observance of Standards and Codes (ROSC) data module was prepared in June 2002 and issued on March 5, 2003. This data module was updated in August 2005.

\section{Management's visit}

At the invitation of the authorities, Mr. Kato, Deputy Managing Director, visited Maputo, Mozambique in July 2005. The Managing Director visited Mozambique on August 2007 for a meeting with the African Consultative Group on Quotas, Voice, and Representation.

\section{Resident representative}

Mr. Victor Lledo has been the IMF's resident representative to Mozambique since June 21, 2010. 


\begin{tabular}{|c|c|c|c|c|}
\hline \multicolumn{5}{|c|}{$\begin{array}{l}\text { IMF Technical Assistance Provided to Mozambique } \\
\qquad(\text { (2006-11) }\end{array}$} \\
\hline Departments & Timing & Form & Purpose & Counterparts \\
\hline Finance & February 2008 & Mission & $\begin{array}{l}\text { Update of Safeguard } \\
\text { Assessment }\end{array}$ & Bank of Mozambique \\
\hline \multirow[t]{17}{*}{ Fiscal Affairs } & March 2011 & Mission & $\begin{array}{l}\text { Public Financial Management } \\
\text { Reforms }\end{array}$ & Ministry of Finance \\
\hline & March 2011 & Peripatetic expert & Public Accounting & Ministry of Finance \\
\hline & $\begin{array}{l}\text { November } \\
2010\end{array}$ & Peripatetic expert & $\begin{array}{l}\text { Public Financial Management } \\
\text { Reforms }\end{array}$ & Ministry of Finance \\
\hline & April-May 2010 & Mission & $\begin{array}{l}\text { Revenue Administration } \\
\text { Reform }\end{array}$ & Ministry of Finance \\
\hline & April 2010 & Mission & $\begin{array}{l}\text { Public Financial Management } \\
\text { Reforms }\end{array}$ & Ministry of Finance \\
\hline & Feb.-Mar. 2010 & Mission & Tax Policy Review & Ministry of Finance \\
\hline & May 2009 & Mission & Tax Administration Reform & Ministry of Finance \\
\hline & April 2008 & Mission & $\begin{array}{l}\text { Public Financial Management } \\
\text { Reforms }\end{array}$ & Ministry of Finance \\
\hline & April-May 2008 & Mission & $\begin{array}{l}\text { Revenue Administration } \\
\text { Reform }\end{array}$ & Ministry of Finance \\
\hline & Oct.-Nov. 2007 & Mission & Tax Administration Inspection & Ministry of Finance \\
\hline & September 2007 & Mission & $\begin{array}{l}\text { Public Private Partnerships } \\
\text { and Fiscal Risks }\end{array}$ & Ministry of Finance \\
\hline & August 2007 & Peripatetic expert & Tax Policy and Administration & Ministry of Finance \\
\hline & August 2007 & Mission & $\begin{array}{l}\text { Fiscal ROSC Update and } \\
\text { Public Financial Management } \\
\text { inspection }\end{array}$ & Ministry of Finance \\
\hline & April-May 2007 & Mission & Tax Administration Inspection & Ministry of Finance \\
\hline & April-May 2007 & Mission & Petroleum Fiscal Regime & $\begin{array}{l}\text { Ministry of Finance } \\
\text { Ministry of Mineral } \\
\text { Resources }\end{array}$ \\
\hline & December 2006 & Mission & Tax Administration & Ministry of Finance \\
\hline & March 2006 & Mission & Tax Policy Review & Ministry of Finance \\
\hline \multirow[t]{3}{*}{$\begin{array}{l}\text { Monetary and } \\
\text { Capital Markets }\end{array}$} & September 2010 & Mission & $\begin{array}{l}\text { Follow-up on Medium-Term } \\
\text { Debt Strategy, Debt Markets, } \\
\text { and Capacity to Conduct Debt } \\
\text { and Project Analysis }\end{array}$ & $\begin{array}{l}\text { Minister of Finance } \\
\text { Bank of Mozambique }\end{array}$ \\
\hline & June 2010 & Mission & $\begin{array}{l}\text { Medium-Term Debt Strategy, } \\
\text { Debt Markets, and Capacity to } \\
\text { Conduct Debt and Project } \\
\text { Analysis }\end{array}$ & $\begin{array}{l}\text { Minister of Finance } \\
\text { Bank of Mozambique }\end{array}$ \\
\hline & April 2010 & Mission & $\begin{array}{l}\text { Strengthening Monetary } \\
\text { Policy Formulation and } \\
\text { Implementation }\end{array}$ & Bank of Mozambique \\
\hline
\end{tabular}




\begin{tabular}{|c|c|c|c|c|}
\hline \multicolumn{5}{|c|}{$\begin{array}{l}\text { IMF Technical Assistance Provided to Mozambique } \\
\qquad(2006-11)\end{array}$} \\
\hline \multirow[t]{13}{*}{ Departments } & Timing & Form & Purpose & Counterparts \\
\hline & November 2009 & Mission & $\begin{array}{l}\text { Strengthening Monetary } \\
\text { Policy Formulation and } \\
\text { Implementation }\end{array}$ & Bank of Mozambique \\
\hline & May 2009 & Mission & 2009 FSAP Follow up & Bank of Mozambique \\
\hline & February 2009 & Mission & $\begin{array}{l}\text { TA on Central Banking and } \\
\text { Monetary Policy Formulation }\end{array}$ & Bank of Mozambique \\
\hline & May 2008 & Mission & $\begin{array}{l}\text { Strengthening Monetary } \\
\text { Policy Formulation and } \\
\text { Implementation }\end{array}$ & Bank of Mozambique \\
\hline & December 2007 & Mission & $\begin{array}{l}\text { Implementation of IFRS for } \\
\text { Central Bank Accounts. }\end{array}$ & Bank of Mozambique \\
\hline & August 2007 & Mission & $\begin{array}{l}\text { Post-FSAP TA Assessment: } \\
\text { Banking supervision, } \\
\text { Monetary Policy and } \\
\text { Operations }\end{array}$ & Bank of Mozambique \\
\hline & April 2007 & $\begin{array}{l}\text { Short-term } \\
\text { consultant }\end{array}$ & Banking Supervision & Bank of Mozambique \\
\hline & October 2006 & Mission & $\begin{array}{l}\text { Post-FSAP TA Assessment: } \\
\text { Banking supervision, } \\
\text { Monetary Policy and } \\
\text { Operations }\end{array}$ & Bank of Mozambique \\
\hline & Feb-March 2006 & Mission & AML/CFT legislation & Bank of Mozambique \\
\hline & Jan-Feb 2006 & $\begin{array}{l}\text { Short-term } \\
\text { consultant }\end{array}$ & Monetary Policy Framework & Bank of Mozambique \\
\hline & Jan-Feb 2006 & $\begin{array}{l}\text { Short-term } \\
\text { consultant }\end{array}$ & Monetary Operations & Bank of Mozambique \\
\hline & January 2006 & $\begin{array}{l}\text { Short-term } \\
\text { consultant }\end{array}$ & Exchange Rate Management & Bank of Mozambique \\
\hline \multirow[t]{8}{*}{ Statistics } & February 2010 & Mission & Multitopic GDDS & $\begin{array}{l}\text { National Institute of } \\
\text { Statistics }\end{array}$ \\
\hline & $\begin{array}{l}\text { March-April } \\
2008\end{array}$ & Mission & National Accounts Statistics & $\begin{array}{l}\text { National Institute of } \\
\text { Statistics }\end{array}$ \\
\hline & February 2008 & Mission & Balance of Payments Statistics & Bank of Mozambique \\
\hline & December 2007 & Mission & Balance of Payments Statistics & Bank of Mozambique \\
\hline & November 2007 & Mission & $\begin{array}{l}\text { Monetary and Financial } \\
\text { Statistics }\end{array}$ & Bank of Mozambique \\
\hline & June-July 2006 & Mission & Consumer Price Statistics & $\begin{array}{l}\text { National Institute of } \\
\text { Statistics (INE) }\end{array}$ \\
\hline & $\begin{array}{l}\text { August 2005- } \\
\text { July } 2006\end{array}$ & $\begin{array}{l}\text { Long-term } \\
\text { consultant }\end{array}$ & National Accounts Statistics & $\begin{array}{l}\text { National Institute of } \\
\text { Statistics }\end{array}$ \\
\hline & July 2005 & Mission & Government Finance Statistics & $\begin{array}{l}\text { National Institute of } \\
\text { Statistics }\end{array}$ \\
\hline
\end{tabular}




\section{APPENDIX II. WORLD BANK-IMF COLLABORATION}

36. The Fund Mozambique team met with the World Bank Mozambique team in Maputo during the 2011 Article IV consultation and second PSI review mission in April 2011. The objective was to identify macro-critical structural reforms and to coordinate the two teams' work for the period May 2011- April 2012.

2. The teams agreed that Mozambique's main macroeconomic challenges are to sustain higher and more inclusive economic growth going forward. To meet these challenges, Mozambique needs to enable broader-based economic activity led by the private sector, in particular in areas where it has competitive advantages, such as agriculture, traderelated services in support of booming commodity export sectors, labor-intensive manufacturing, and tourism. Given the low level of infrastructure, this will entail policies and reforms to boost public investment, coupled with enhancements in the business environment. While traditional aid will continue to address needs in infrastructure investment and the social sector, increasing public investment may require resorting to nonconcessional financing and stepping up capacity in the investment selection and debt management areas.

3. Based on this shared assessment, the teams identified six structural reform areas as macro-critical, in view of their central role in achieving higher and more inclusive growth:

Debt management and investment planning. The authorities' ambitious investment program will need to be backed by a strong reform agenda to enhance capacity to manage fiscal risks and ensure value for money. In the field of investment selection, several measures will be implemented to establish an investment pipeline and define project selection standards and protocols. A new legal framework supporting private-public partnership and concessions will be implemented. Debt management will be strengthened to ensure that borrowing and investment decisions entail value for money. This will be underpinned by a new medium-term debt strategy.

Public financial management. The focus will be on enhancing the capacity to manage risks outside the central government, including in the area of state-owned enterprises (SOEs) and decentralized administrative units. To achieve this objective, the authorities will step up riskbased controls and audits, continue rolling out their new PFM system, and enhance budget execution and monitoring reports. Other critical measures aim at enhancing control of the wage bill, both in budget preparation and execution. These reforms are macro-critical since they will create fiscal space needed to reduce poverty and increase priority spending, while managing risks related to SOE's large-scale investment plans and enhancing the government's oversight.

Tax administration. Despite the strong increase in revenue collections, the tax system is still complex to administer and comply with. It is marked by numerous tax benefits and an overall low contribution from the mega-project sectors. Going forward, the government plans to facilitate tax compliance through electronic tax payment and continue to expand the tax base to cover the large informal sector. The revenue management system will be enhanced, in line with a set of agreed performance indicators. The large tax-payers unit will be made fully operational.

Social safety nets. The recent social unrest in light of the food and fuel price spikes has exposed the contrast of a fast-growing economy with still large pockets of poverty. This, in turn, 
undermines the country's long-term growth potential. To address this, the authorities intend to strengthen their safety nets. Measures include: (i) rationalizing and expanding health insurance coverage; (ii) strengthening higher education and vocational training; (iii) strengthening the financial sustainability and administration of the National Social Security Fund, and creating a new pension system for private sector workers; and (iv) improving the targeting of social protection systems to the most needy. These measures are macro-critical, as better health and education services, stronger pensions systems, and a more effective social safety net can enhance the productivity of the workforce and support social cohesion.

Investment climate. Mozambique's corporate sector is held back by the high costs of doing business. These arise mainly from weaknesses in governance, access to finance, power supply, tax and regulatory systems, and contract enforcement. Wide-ranging reforms are needed to address these issues. They are macro-critical because a better investment climate is conducive to supporting private sector activities, thereby broadening the country's productive and export base, creating jobs, and thus helping reduce poverty.

Financial sector stability and development. Credit to the private sector has strongly expanded during the last few years, and there are concerns about the impact on the quality of the loans. A number of measures are envisaged to strengthen the supervision of the financial sector, as well as the capacity to identity and address potential sources of vulnerabilities. Meanwhile, improving financial intermediation and access to credit remains a top priority. To this end, the authorities will finalize, by end-September 2011, the 2011-20 Financial Sector Development Strategy which will include a wide-ranging and clearly sequenced action plan, covering ways to, among other things, enhance financial inclusion, competition, consumer protection, and financial literacy.

\section{The teams agreed on the following division of labor:}

Debt management and investment planning reforms. The Fund will continue to play a key role in building government capacity to assess debt sustainability and define a coherent debt strategy through TA and continued support from HQ. Both institutions will assist the authorities in strengthening their debt management capacity, with TA support from the Fund and on-site assistance from the Bank. The Fund will continue to play a key role in helping the authorities establish a project selection pipeline, in line with recent TA and program commitments, while the Bank will take a lead in enhancing project evaluation and monitoring processes and protocols from its office in Mozambique, in consultation with the Fund. The Fund review missions will provide further opportunities to assess progress in this field and discuss possible actions with the Bank.

Public financial management reform. The Band and the Fund will cooperate in the field of rolling out the new PFM system to cover local administrations and SOEs engaging in large-scale infrastructure investments. The Fund will secure its support through regular TA missions from its RTAC office. The Bank will provide ongoing consultation from its local office and supervision missions of projects under implementation in this area. The Fund program review missions will provide further opportunities to assess progress in this field.

Tax administration reforms. The Fund will provide support through ongoing advice based on regular TA. The Bank will advance reforms in this area in the context of its business environment policies and portfolio. 
Social safety net reform. The Bank will take a lead in this field through continued TA in the design of more comprehensive and performing safety nets, in cooperation with other development partners and international organizations (including UNESCO and ILO). The Fund will play its role in identifying the fiscal space for the safety nets. The results will be discussed among the collaborators on a regular basis, so as to inform policy discussions with the authorities.

Investment climate reform. The Bank will take a lead in enhancing the investment climate by deepening its agenda to cut red tape and simplifying the legal framework. The results will be shared on an ongoing basis and discussed during the Fund program review missions.

Financial sector stability and development. The Fund will continue to help the Bank of Mozambique to build its capacity to assess risks to financial stability of the banking sector in upcoming missions, while the Bank will take the lead in supporting financial sector development and reforms, including through enhanced financial inclusion, competition, consumer protection, and financial literacy. The results of their respective work will be shared and discussed regularly.

\section{The teams have the following requests for information from their counterparts:}

- $\quad$ The Fund team requests to be kept informed of progress in the above macro-critical structural reform areas. Timing: when milestones are reached; and at least semiannually during Fund program review missions.

- $\quad$ The Bank team requests to be kept informed of the Fund's assessments of macroeconomic policies and prospects, and analytical work on areas under the Fund's lead, as well as on recommendations of Fund TA missions. Timing: in the context of Fund program review and other missions, and at least semi-annually.

6. The table below lists the teams' separate and joint work programs during May 2011- April 2012. 


\begin{tabular}{|c|c|c|c|}
\hline \multicolumn{4}{|c|}{ Mozambique: Bank and Fund planned activities in macro-critical structural reform areas, 2011-12 } \\
\hline Title & Products & Timing of Missions & Expected delivery date \\
\hline 1. Bank Work & $\begin{array}{l}\text { FSTAP project support to financial sector reforms } \\
\text { Updates on advancing structural reforms: procurement, } \\
\text { decentralization, business environment, financial sector } \\
\text { Establishing a collection of relevant papers in the field of "Inclusive } \\
\text { Growth" in light of the Indy Village Workshop } \\
\text { Recommendations on targeted, sustainable safety net measures } \\
\text { (including conditional cash transfer system) }\end{array}$ & $\begin{array}{l}\text { Ongoing } \\
\text { Ongoing } \\
\text { February } 2010 \\
\text { Ongoing }\end{array}$ & $\begin{array}{l}\text { Ongoing } \\
\text { June } 2010 \text { to June } 2011 \\
\text { May } 2011 \\
\text { December } 2012\end{array}$ \\
\hline $\begin{array}{l}\text { 2. Fund Work } \\
\text { program }\end{array}$ & $\begin{array}{l}\text { Second PSI Review and } 2011 \text { Article IV } \\
\text { Third PSI Review } \\
\text { Updated macro framework data and PSI review staff report } \\
\text { TA follow-up missions on revenue administration } \\
\text { TA follow-up mission on PFM } \\
\text { TA follow-up mission on tax policy } \\
\text { TA on SISTAFE } \\
\text { TA on budget execution } \\
\text { TA on payment system oversight } \\
\text { TA on banking supervision and regulation } \\
\text { TA on monetary policy formulation within an inflation targetting } \\
\text { framework } \\
\text { TA follow-up mission on medium term debt strategy } \\
\text { TA on BOP/IIP statistics } \\
\text { TA follow-up on GFS to improve fiscal data in line with GFSM } \\
2001\end{array}$ & $\begin{array}{l}\text { March } 2011 \\
\text { October } 2011 \\
\text { PSI reviews } \\
\text { April 2011, August 2011, } \\
\text { February } 2012 \\
\text { April } 2011 \\
\text { May } 2011 \\
\text { September 2011, February } 2012 \\
\text { March 2012 } \\
\text { May 2011, November } 2011 \\
\text { May 2011 } \\
\text { May - August 2011, December } \\
2011 \\
\text { January 2012 } \\
\text { June 2011, December } 2011 \\
\text { March 2012 }\end{array}$ & $\begin{array}{l}\text { June } 2011 \\
\text { December } 2011 \\
\text { April } 2011 \text { - December } 2011 \\
\text { April } 2012 \\
\text { June } 2011 \\
\text { July } 2011 \\
\text { April } 2012 \\
\text { May } 2012 \\
\text { January } 2012 \\
\text { June } 2011 \\
\text { February } 2012 \\
\text { March } 2012 \\
\text { February } 2012 \\
\text { May } 2012\end{array}$ \\
\hline $\begin{array}{l}\text { 3. Joint Work } \\
\text { Program }\end{array}$ & $\begin{array}{l}\text { PFM: update of work plan for 2009-11 in light of findings from } \\
\text { PEFA and ROSC } \\
\text { PFM: continue to monitor consolidation and expansion of e- } \\
\text { SISTAFE. } \\
\text { Policy advice on public sector salary reforms (incl. pensions) } \\
\text { Review of tax policy } \\
\text { JSAN on the authorities' new Poverty Reduction for 2011-14 }\end{array}$ & $\begin{array}{l}\text { March } 2011 \\
\text { Ongoing } \\
\text { Ongoing } \\
\text { TBD }\end{array}$ & $\begin{array}{l}\text { March } 2011 \\
\text { Ongoing } \\
\text { Ongoing } \\
\text { TBD } \\
\text { August } 2011\end{array}$ \\
\hline
\end{tabular}




\section{APPENDiX III. MozambiQue: Statistical ISSUES}

\section{Assessment of Data Adequacy for Surveillance}

General: Data provision has some shortcomings, but is broadly adequate for surveillance. Despite the increase in budget resources allocated to the compilation of official statistics, continued high reliance on external funding raises concerns about the sustainability of the programs of the National Institute of Statistics (INE). The authorities are making efforts to improve the quality and timeliness of economic and financial data through participation in the Fund's General Data Dissemination System (GDDS) and in the external sector module of the GDDS Project for Anglophone African Countries (funded by the U.K. Department for International Development (DFID)). Weaknesses exist, particularly in the areas of national accounts, prices, and government finance statistics.

Real sector statistics: The national accounts are prepared by the INE in accordance with the 1993 System of National Accounts (1993 SNA). In August 2007, the INE released quarterly GDP estimates at constant prices covering 2000-07 for the first time, along with a revised annual GDP series for the period 1991-2006, with 2003 as the new base year. The authorities are working to strengthen quarterly national accounts compilation at current prices. The INE's new household income and expenditure survey and economic censuses leading to a new business registry still need to be incorporated to strengthen the national accounts. A revamped consumer price index for Maputo based on weights derived from the 2008-09 household survey was released in February 2011. A national index obtained by integrating the indices for Maputo, Beira, and Nampula was released in April 2006. INE has adopted a new software from INE Portugal which helps to correct a probable downward bias caused by carry-forward techniques for discontinued products. Several issues remain outstanding, however, including how to improve the housing market rent sample and expand coverage to include the Quelimane and Greater Maputo areas. There are insufficient sectoral labor market and employment data, and where available, they have limited coverage. A oneyear labor market survey of the entire country, undertaken by INE in collaboration with the Labor Ministry, was launched in October 2004.

Government finance statistics: Government finance statistics (GFS) are not compiled using an internationally accepted statistical methodology and no staff has been identified to do so. A complete list of public sector entities is lacking. Work on a correspondence table between budgetary information and GFS needs to be completed. In addition, work on an integrated information system to capture completely stocks and flow information needs further development. 
Monetary and financial statistics: A 2004 work plan was developed to implement the Monetary and Financial Statistics Manual (MFSM) and develop an integrated monetary database (IMD) to meet the needs of the Bank of Mozambique (BM), AFR, and STA. In November 2007 the IMD was completed and allows the derivation of accurate and timely monthly monetary statistics, while reducing BM's reporting burden.

Balance of payments: With assistance from STA, provided in the context of the GDDS regional project, the $\mathrm{BM}$ has made significant progress toward compiling and disseminating balance of payments (BOP) and international investment position (IIP) statistics that are fully aligned with the Balance of Payments Manual, fifth edition (BPM5). The BM has an adequate institutional framework for the compilation of BOP and IIP statistics. However, further strengthening of external sector statistics needs to focus on: strengthening information technology (especially to apply data warehouse and on-line analytical processing tools); excluding the effects of price and exchange rate fluctuations from reserve asset flows; applying a methodology (provided through STA technical assistance) to estimate reinvested profits and stocks of financial assets and liabilities held abroad by the nonfinancial public sector; improving business surveys; publishing methodological notes, in general, and more data covering the Reserves Template, foreign direct investment, goods trade and current transfers; and beginning the gradual process to implement the new Balance of Payments and International Investment Position Manual, sixth edition. Joint work with other institutions to improve the quality of external trade data, especially with regard to price and volume indices, also merits attention.

\section{Data Standards and Quality}

Mozambique commenced its participation in the General Data Dissemination System (GDDS) in November 2003; but has since then not updated much of its metadata.

In May 2005, an update of the June 2002 data module of the Report on the Observance of Standards and Codes (Data ROSC) was prepared. Improvements in the institutional environment and increased allocation of resources for the compilation of national accounts and balance of payments statistics aimed to address weaknesses in the prerequisites for the quality of the statistics. Methodological soundness, accuracy, and reliability of macroeconomic statistics began to show improvements as a result of these efforts. 


\section{Reporting to STA}

Mozambique does not report fiscal data for publication in the IFS or the Government Finance Statistic Yearbook (GFSY). Mozambique now reports monthly monetary data to STA using the standardized reporting forms (SRFs). As a result, these enhanced data are being published in the International Financial Statistics (IFS) Supplement. In 2007, the BM started to compile and report comprehensive IIP data in the Balance of Payments Statistics Yearbook. 


\section{Mozambique: Table of Common Indicators Required for Surveillance}

(As of April 30, 2011)

${ }^{1}$ Includes reserve assets pledged or otherwise encumbered as well as net derivative positions.

${ }^{2}$ Both market-based and officially determined, including discount rates, money market rates, rates on treasury bills, notes and bonds.

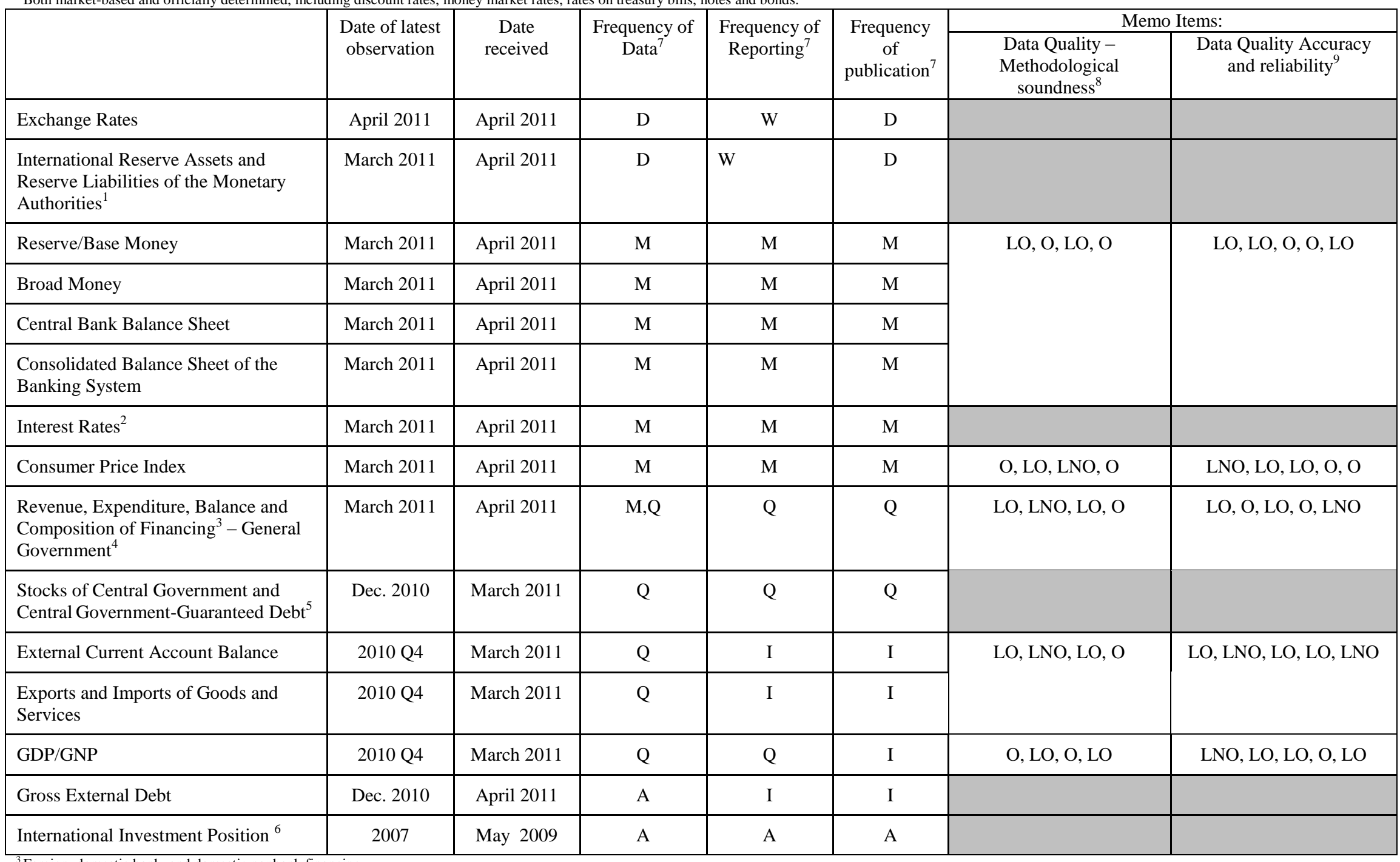

${ }^{3}$ Foreign, domestic bank, and domestic nonbank financing.

${ }^{4}$ The general government consists of the central government (budgetary funds, extrabudgetary funds, and social security funds) and state and local governments.

${ }^{5}$ Including currency and maturity composition.

${ }^{6}$ Includes external gross financial asset and liability positions vis-à-vis nonresidents.

7 Includes external gross financial asset and liability positions vis-à-vis nonresidents.

${ }^{8}$ Daily (D), Weekly (W), Monthly (M), Quarterly (Q), Annually (A), and Not Available (NA).

${ }^{9}$ Reflects the assessment provided in the data ROSC or the Substantive Update for the dataset corresponding to the variable in each row. The assessment indicates whether international standards concerning concepts and definitions, scope,

classification/sectorization, and basis for recording are fully observed (O); largely observed (LO); largely not observed (LNO); not observed (NO); and not available (NA). 


\section{INTERNATIONAL MONETARY FUND}

EXTERNAL

Public Information Notice

RELATIONS

DEPARTMENT

Public Information Notice (PIN) No. 11/82

FOR IMMEDIATE RELEASE

June 27, 2011

International Monetary Fund

$70019^{\text {th }}$ Street, NW

Washington, D. C. 20431 USA

\section{IMF Executive Board Concludes 2011 Article IV Consultation with Mozambique}

On June 17, 2011, the Executive Board of the International Monetary Fund (IMF) concluded the Article IV consultation with Mozambique. ${ }^{1}$

\section{Background}

Mozambique has sustained strong economic growth over nearly two decades, helped by foreign investment, mainly in mega-projects in the natural resource sector, and generous support by development partners. However, trend growth has declined in recent years and poverty reduction stagnated, suggesting the need for building more inclusive growth through economic diversification, employment creation, and the expansion of social protection.

Mozambique has weathered the adverse shocks of recent years relatively well. Economic growth remains one of the highest in the region, partially reflecting the authorities' supportive policy stance during the global crisis. Growth in 2010 is estimated at $61 / 2$ percent, supported by external demand. Inflation, even excluding food and fuel, strongly picked up and reached double digits, triggered by the accommodating monetary policy adopted during the crisis and a depreciating exchange rate during much of the year. The current account deficit narrowed more than expected, supported by buoyant mega-projects exports. Strong revenue collections, reflecting tax administration efforts, and spending restraint helped contain the fiscal deficit in 2010 , in spite of the high costs of the fuel subsidy which put pressures on domestic spending.

\footnotetext{
${ }^{1}$ Under Article IV of the IMF's Articles of Agreement, the IMF holds bilateral discussions with members, usually every year. A staff team visits the country, collects economic and financial information, and discusses with officials the country's economic developments and policies. On return to headquarters, the staff prepares a report, which forms the basis for discussion by the Executive Board. At the conclusion of the discussion, the Managing Director, as Chairman of the Board, summarizes the views of Executive Directors, and this summary is transmitted to the country's authorities. An explanation of any qualifiers used in summings up can be found here: http://www.imf.org/external/np/sec/misc/qualifiers.htm.
} 
Mozambique's medium-term outlook remains favorable. Economic growth is projected to accelerate to $71 / 4$ percent in 2011 and close to 8 percent over the medium term. This mainly reflects strong new mega-project activity and stepped-up public investment. Headline inflation is projected to decline to single digits as a result of appropriately tight macroeconomic policies, while Mozambique's external position should remain comfortable. Nonetheless, continued spillovers from international food and fuel prices could be a drag on macroeconomic and social stability.

Significant policy challenges remain over the medium term. The new Poverty Reduction Strategy aims to respond to these challenges and create the conditions for high, sustained, and inclusive economic growth. This includes policies to increase production and productivity in labor-intensive sectors, such as agriculture, and promoting employment through improvements in the business environment. Private sector activity would benefit from a larger contribution of the financial sector. Fiscal space needs to be created to finance large public investment and support an expansion of social protection, while safeguarding macroeconomic stability and debt sustainability. This will hinge on enhancing government revenues, safeguarding donor support, pursuing a prudent borrowing strategy, and stepping up debt management and investment planning. Continued improvements in public financial management will facilitate expenditure prioritization and execution.

\section{Executive Board Assessment}

Executive Directors agreed with the thrust of the staff appraisal. They commended the authorities for prudent policies that have resulted in a strong economic performance. Directors noted that, although Mozambique's medium-term prospects continue to be favorable, significant challenges remain in managing macroeconomic risks and ensuring that the benefits from growth are shared fairly across the population.

Directors underscored that safeguarding price stability and containing inflation expectations remain key policy priorities for the period ahead. In this regard, they supported the authorities' intention to tighten fiscal and monetary policies as needed to bring about an early and sustained decline in core inflation.

Directors welcomed the authorities' new Poverty Reduction Strategy (PARP). They supported the goals of broadening the country's productive and export base, creating additional employment opportunities, and expanding the social safety nets. Continued structural reforms to improve productivity and the business environment will be important to achieve sustained growth and poverty reduction. Directors encouraged the authorities to seek the expertise of development partners in designing well-targeted social protection systems.

Directors supported the authorities' prudent fiscal stance aimed at preserving macroeconomic stability and debt sustainability. They welcomed the authorities' intention to gradually phase out 
the fuel subsidy. With a view to creating fiscal space, Directors encouraged the authorities to sustain efforts to improve tax administration and seek new revenue sources. In particular, they encouraged the authorities to adopt a prudent approach to generating additional budgetary revenue from the natural resource sector while protecting Mozambique as a destination for foreign investment. Directors emphasized the importance of adhering to a prudent borrowing strategy, further strengthening debt management, and improving investment planning.

Directors noted the resilience of the banking system during the global crisis, and recommended continued vigilance in light of remaining vulnerabilities. In particular, Directors stressed the importance of adequate capital buffers at all banks, better diversified loan books, and a strict oversight of financial flows between banks and their parent companies abroad. They welcomed progress in establishing a crisis resolution framework and enhancing bank supervision, while noting the need for steps to make the Anti-Money Laundering and Combating the Financing of Terrorism (AML/CFT) framework more effective.

Directors welcomed the authorities' acceptance of the obligations under Article VIII of the Fund's Articles of Agreement.

It is expected that the next Article IV consultation with Mozambique will be held in accordance with the Executive Board decision on consultation cycles for members with Fund arrangements.

Public Information Notices (PINs) form part of the IMF's efforts to promote transparency of the IMF's views and analysis of economic developments and policies. With the consent of the country (or countries) concerned, PINs are issued after Executive Board discussions of Article IV consultations with member countries, of its surveillance of developments at the regional level, of post-program monitoring, and of ex post assessments of member countries with longer-term program engagements. PINs are also issued after Executive Board discussions of general policy matters, unless otherwise decided by the Executive Board in a particular case. 
Mozambique: Selected Economic and Financial Indicators, 2009-12

$\begin{array}{rrrr}2009 & 2010 & 2011 & 2012 \\ \text { Act. } & \text { Est. } & \text { Proj. } & \text { Proj. }\end{array}$

(Annual percentage change, unless otherwise indicated)

National income and prices

Nominal GDP (MT billion)

Nominal GDP growth

Real GDP growth

GDP per capita (US\$)

Consumer price index (annual average)

Consumer price index (end of period)

Exchange rate, MT per US dollar, eop

Exchange rate, MT per US dollar, per.avg.

External sector

Merchandise exports

Merchandise exports, excluding megaprojects

Merchandise imports

Merchandise imports, excluding megaprojects

Terms of trade

Nominal effective exchange rate (end of period)

Real effective exchange rate (end of period)

Money and credit

Reserve money

M3 (Broad Money)

Credit to the economy

(Percent of GDP)

Investment and saving

Gross domestic investment

Government

Other sectors

Gross domestic savings (excluding grants)

Government

Other sectors

External current account, before grants

External current account, after grants

Government budget

Total revenue

Total expenditure and net lending

Overall balance, before grants

Total grants

Overall balance, after grants

Domestic primary balance, before grants

External financing (incl. debt relief)

Net domestic financing

Privatization

Total public debt

Of which: external

Of which: domestic

External current account, before grants

External current account, after grants

Overall balance of payments

Net international reserves (end of period) ${ }^{1}$

Gross international reserves (end of period) ${ }^{1}$

Months of projected imports of goods and nonfactor services $\begin{array}{llll}269 & 323 & 380 & 439\end{array}$

$\begin{array}{llll}12.1 & 20.1 & 17.4 & 15.7\end{array}$

$\begin{array}{llll}6.3 & 6.6 & 7.2 & 7.5\end{array}$

$\begin{array}{llll}477 & 454 & 550 & 603\end{array}$

$\begin{array}{llll}3.3 & 12.7 & 9.5 & 7.2\end{array}$

$\begin{array}{llll}4.2 & 16.6 & 8.4 & 5.6\end{array}$

$\begin{array}{llll}27.5 & 32.8 & \ldots & \ldots\end{array}$

$\begin{array}{rrrr}-19.1 & 4.5 & 14.8 & 17.3 \\ 4.3 & -31.3 & 6.9 & 3.4 \\ -6.1 & -2.5 & 16.1 & 10.8 \\ -10.6 & -7.4 & 16.4 & 12.6 \\ -0.7 & 15.0 & 4.9 & 5.2 \\ -7.1 & -22.4 & \ldots & \ldots \\ -6.3 & -15.0 & \ldots & \ldots\end{array}$

$\begin{array}{llll}27.3 & 29.2 & 14.3 & 16.9\end{array}$

$\begin{array}{llll}32.6 & 22.8 & 20.0 & 23.1\end{array}$

$\begin{array}{llll}58.6 & 27.5 & 19.5 & 20.0\end{array}$

$\begin{array}{llll}26.5 & 28.2 & 28.6 & 29.7\end{array}$

(Percent of GDP)

$\begin{array}{rrrr}16.3 & 20.6 & 21.3 & 21.5 \\ 12.8 & 12.6 & 11.7 & 11.4 \\ 3.5 & 8.0 & 9.6 & 10.1 \\ -2.6 & 4.3 & 4.3 & 5.3 \\ -0.4 & 1.2 & 1.0 & 0.8 \\ -2.2 & 3.1 & 3.3 & 4.5 \\ -18.9 & -16.4 & -17.0 & -16.2 \\ -12.1 & -10.2 & -11.4 & -10.9\end{array}$

$\begin{array}{llll}17.4 & 19.6 & 20.2 & 20.6\end{array}$

$\begin{array}{llll}32.2 & 31.4 & 33.9 & 34.4\end{array}$

$\begin{array}{llll}-14.8 & -11.8 & -13.7 & -13.8\end{array}$

$\begin{array}{llll}9.4 & 8.1 & 7.3 & 7.1\end{array}$

$\begin{array}{llll}-5.4 & -3.7 & -6.4 & -6.8\end{array}$

$\begin{array}{llll}-4.3 & -3.2 & -3.1 & -3.4 \\ 5.0 & -4.3 & 5.8 & 5.8\end{array}$

$\begin{array}{llll}5.0 & 4.3 & 5.8 & 5.8\end{array}$

$\begin{array}{llll}0.2 & -0.5 & 0.5 & 1.0\end{array}$

$\begin{array}{llll}0.2 & 0.0 & 0.0 & 0.0\end{array}$

$\begin{array}{llll}38.2 & 39.3 & \ldots & \ldots\end{array}$

$\begin{array}{rrrr}35.4 & 35.2 & \ldots & \ldots \\ 2.8 & 4.1 & \cdots\end{array}$

(US\$ millions, unless otherwise indicated)

$\begin{array}{rrrr}-1,908 & -1,603 & -2,055 & -2,195 \\ -1,220 & -999 & -1,380 & -1,478 \\ 194 & 58 & 91 & 302 \\ 1,840 & 1,908 & 2,000 & 2,302 \\ 2,012 & 2,099 & 2,189 & 2,487 \\ 5.4 & 4.8 & 4.5 & 4.7\end{array}$

Sources: Mozambican authorities; and IMF staff estimates and projections.

${ }^{1}$ Includes disbursements of IMF resources under the ESF and August 2009 SDR allocation. 


\section{IMF Executive Board Completes Second Review Under Policy Support Instrument for Mozambique}

The Executive Board of the International Monetary Fund (IMF) today completed the second review of Mozambique's economic program under the Policy Support Instrument (PSI). In completing the review, the Board approved a waiver for the nonobservance of the quantitative assessment criterion on reserve money.

The Executive Board approved Mozambique's second three-year PSI on June 14, 2010 (see Press Release No. 10/242). The IMF's framework for PSIs is designed for low-income countries that may not need, or want, IMF financial assistance, but still seek IMF advice, monitoring, and endorsement of their policies (see Public Information Notice No. 05/145).

At the conclusion of the Executive Board discussion on Mozambique, Ms. Nemat Shafik, Deputy Managing Director and Acting Chair, made issued the following statement:

“Mozambique's economic performance and outlook remain strong. Helped by appropriate macroeconomic policies, the economy proved resilient to the global economic crisis. The country is poised for an acceleration of economic growth over the medium term, reflecting expanded production in the natural resource sector and stepped-up public infrastructure investment.

"Fighting inflation should be the key priority for macroeconomic policy in the short run. The authorities' policies aimed at bringing about an early decline in core inflation are welcome, and the authorities should stand ready to tighten policies further should inflation pressures persist.

"The new Poverty Reduction Strategy (PARP) contains the right ingredients to allow growth to become more inclusive. The PARP's emphasis on broadening the country's productive and export base and creating employment opportunities is appropriate, but determination is needed in implementing the strategy. The authorities' commitment to continue the phase-out of the fuel subsidy is welcome, and they should move forward in expanding well-targeted and affordable social protection systems. 
“The authorities' ambitious development agenda appropriately addresses Mozambique's economic challenges. In implementing the investment strategy, it will be important to continue to pursue a prudent borrowing policy to safeguard macroeconomic stability and debt sustainability. Efforts to strengthen tax administration should be sustained and new revenue sources tapped, including in particular from the natural resource sector. The authorities are also well advised to continue implementing key structural reforms in debt management and investment planning, as well as public financial management.

"While the banking system has shown resilience during the global crisis, vigilance is warranted in light of vulnerabilities and potential contagion. The progress in establishing a banking crisis resolution framework and improving banking supervision is welcome, while more resolve needs to be shown to make the Anti-Money Laundering/Combating the Financing of Terrorism framework more effective.

"The acceptance of the obligations of Article VIII of the IMF's Articles of Agreement is welcome," she added. 


\section{Statement by Mr. Moeketsi Majoro, Executive Director for Mozambique June 17, 2010}

\section{Introduction}

My authorities are grateful to Fund management for their continuous support and to staff for constructive policy dialogue and guidance against the backdrop of a very challenging global economic environment. The authorities share the thrust of the staff report as it provides a forthright assessment of recent economic developments and program performance.

\section{Recent Economic Developments}

Despite the uncertain external environment, economic developments in 2010 were characterized by strong growth, strengthened balance of payments and better-than-expected fiscal performance. The Mozambican economy proved resilient boosted by significant exports in mineral mega-projects and infrastructure investment. Real GDP grew at 6.6 percent in 2010, higher than that of its regional peers. However, despite robust efforts by the authorities to tighten monetary and fiscal policies, inflation reached double digits for the first time in a decade, fueled by global food and fuel price increases.

\section{Program Performance}

Program performance has been strong. All end-December 2010 quantitative assessment criteria (AC) were observed, with the exception of the target on reserve money for which the authorities have since taken corrective action to rein in its growth. On the structural front, all benchmarks were implemented. In this context, my authorities request Directors' support for the completion of the second review under the Policy Support Instrument, and for modification of assessment criteria as outlined in the staff report. My authorities remain committed to prudent macroeconomic policies and maintaining strong program implementation going forward.

\section{Economic outlook}

Reducing the current level of poverty, improving social safety nets, expanding infrastructure investment, and boosting key sectors such as agriculture, fishing, minerals, tourism and transport are key priorities for the Mozambican authorities. However, they are caught between supporting sustainable economic growth and arresting inflation expectations in the context of a challenging internal and external environment. For 2011, my authorities foresee a positive macroeconomic outlook as real GDP is projected to grow by $71 / 4$ percent. They are committed to tightening fiscal and monetary policies in order to control liquidity and monitor reserve money developments aimed at reducing headline inflation to single digits from 2011 onwards. The external current account deficit is projected to stabilize during the next few years, while reserves coverage should approach 5 months of projected imports, fully supported by exports from new mega projects in the mining sector, and continued external support from development partners. 


\section{Poverty Reduction and Growth Strategy}

In May 2011, my authorities adopted a new four-year Poverty Reduction Strategy aimed at reducing poverty from about 55 percent to 42 percent by 2014. The program is focused on three pillars: i) increasing production and productivity in sectors such as agriculture and fisheries; ii) creating employment through improvements in the business climate; and iii) pursuing human and social developments. The Government is committed to promoting long-term economic growth, and in this regard, intends to create the necessary fiscal space, including from improving collection of revenues from the natural resource sector in order to finance infrastructure investment and expand social safety nets. In this regard, they plan to build on the existing National Basic Social Security Strategy (ENSSB), Strategic Program for the Reduction of Urban Poverty (PERPU) and the ongoing national policy dialogue to refine their strategy for social protection.

\section{Fiscal Policy}

The 2011 fiscal objective is to limit the primary domestic fiscal deficit to 3 percent of GDP, $1 / 4$ percent lower than initially projected. The government will continue to target a lower fiscal deficit through prudent expenditure execution and continued buoyancy in revenue collection. However, in line with changing social spending priorities, the government has submitted to Parliament a revised budget incorporating adjustments in the budgetary ceiling for transfers and subsidies to accommodate an existing fuel subsidy and planned outlays to cushion the effects of increases in food and fuel prices on the vulnerable groups. My authorities would like to affirm that the previous measures will be gradually phased out as better targeted and sustainable interventions are implemented. In addition, government will continue to reflect the costs of any government outlays for fuel products in the budget, in line with the best fiscal transparency practices.

Mozambique has a low risk of debt distress as shown in the latest Debt Sustainability Analysis (DSA). My authorities reiterate that fiscal policy will continue to be geared toward safeguarding macroeconomic stability and debt sustainability. In this regard, they will move carefully in making use of the ceiling on nonconcessional borrowing under the program. They will continue to improve their debt management and investment planning, in line with commitments under the program.

\section{Monetary and Financial Sector prospects}

Bringing inflation to single digits is a key priority for the authorities. With average inflation surging to around 12.7 percent in 2010, the Central Bank of Mozambique intends to take measures to contain it at $91 / 2$ percent in 2011 and to reduce it further in the years ahead by continuing to decelerate the pace of monetary expansion. The authorities are committed to maintaining the flexible exchange rate regime and the exchange rate looks broadly in line with macroeconomic fundamentals. On May 20, 2011, my authorities accepted the obligations under Article VIII of the Fund's Articles of Agreement and are on track to remove all identified exchange restrictions shortly. 
Financial sector reform will be guided by the prospective 2011-20 Financial Sector Development Strategy that will be finalized no later than end-September 2011. The BM will continue to monitor the banking system closely to safeguard its soundness, enhance financial competition, and protect consumers. In particular, work will continue to fully develop a financial sector contingency plan, migrate to Basel II, introduce deposit insurance, broaden electronic banking and strengthen defenses against money laundering.

\section{Structural Reforms}

My authorities are committed to continue with the reforms in public financial management (PFM) as well as improving their reporting, monitoring, and control of budget execution. They will continue to improve the e-SISTAFE budget execution system and will roll it out nationally with the aim of completion by 2015. My Mozambican authorities are aware that enhanced debt management and investment planning are critical to ensure prudent borrowing decisions. Thus, they will continue reforming debt management and investment planning through the strengthening of the new Debt Management Committee (DMC) and Project Coordination Committee (PCC) that will oversee project cycle management and borrowing options.

In addition, the authorities intend to pursue a more business-friendly environment through tax policy and administration reforms. They will step up efforts to improve automated revenue collection and better monitoring of large taxpayers through strengthening the existing large taxpayers unit, and lowering transactions costs for taxpayers. Furthermore, an Anti-Corruption Strategy was approved in January 2011, and the authorities intend to submit to Parliament by end-July 2011, the legislation required to implement the strategy. 